\title{
Active Anisotropic Colloids from Colloidal Monolayers
}


Cover: Impression of the phase transition observed in monolayers of colloidal superballs upon varying their shape parameter and a smiley-shaped particle prepared using lithography

ISBN: 978-90-393-7382-8 


\title{
Active Anisotropic Colloids from Colloidal Monolayers
}

\author{
Actieve Anisotropische Collö̈den \\ uit Collö̈dale Monolagen \\ (met een samenvatting in het Nederlands)
}

Proefschrift

ter verkrijging van de graad van doctor aan de Universiteit Utrecht op gezag van de rector magnificus, prof. dr. H.R.B.M. Kummeling, ingevolge het besluit van het college voor promoties in het openbaar te verdedigen op maandag 14 juni 2021 des middags te 2.15 uur

door

Daniël Nathan ten Napel

geboren op 25 maart 1991 te Amsterdam 
Promotor: Prof. dr. A.P. Philipse

Copromotor: Dr. A.V. Petukhov

The research described in this thesis was financially supported by the Nederlandse Organisatie voor Wetenschappelijk Onderzoek (NWO). 


\section{Contents}

$\begin{array}{ll}\text { Chapter 1: General Introduction } & 7\end{array}$

Chapter 2: General Experimental Methods 23

Chapter 3: $\quad$ Form Factor Effects in Colloidal Superball Monolayers 35

Chapter 4: Mechanical Assembly of Colloidal Superball Monolayers 51

$\begin{array}{lll}\text { Chapter 5: } & \text { Active Colloidal Circlers and Spinners } & 71\end{array}$

Chapter 6: $\quad$ Production of Active Anisotropic Hydrogel Particles 87

$\begin{array}{lr}\text { Summary } & 99\end{array}$

$\begin{array}{ll}\text { Samenvatting voor Iedereen } & 103\end{array}$

$\begin{array}{ll}\text { Dankwoord } & 109\end{array}$

$\begin{array}{ll}\text { List of Publications } & 111\end{array}$

$\begin{array}{ll}\text { About the Author } & 113\end{array}$ 



\section{General Introduction}

\section{Abstract}

In this introductory chapter we provide the background, motivation and goals of the research described in this thesis. We briefly review the known literature on colloidal self-assembly and active matter with a focus on anisotropic active colloids and their preparation methods. We share our vision and the future prospects and applications of these anisotropic active colloids and their self-assembled structures. Furthermore, we review the known work on the assembly of colloidal superballs into two-dimensional lattices and discuss how the shape of these colloidal superballs influences the resulting lattice structures. This chapter ends with providing the outline of this thesis. 


\subsection{Colloids and self-assembly}

Colloids are small particles that are roughly between $1 \mathrm{~nm}$ and $1 \mu \mathrm{m}$ in size. The term colloid was coined by Thomas Graham in 1861 and comes from the Greek words 'kolla', meaning glue, and 'eidos', meaning like, after he noticed that dissolved starch was not able to pass through the pores of paper. $\mathrm{He}$ concluded that starch must consist of particles that are larger in size than those of salt, as the dissolved salt was able to pass through the pores [1]. An important characteristic property of particles in this size range is that they display the so-called Brownian motion. Brownian motion is the random motion of particles that results from thermal fluctuations. Brownian motion was discovered by and named after the botanist Robert Brown who first described this phenomenon in 1827 after he observed small particles displaying seemingly random motion while studying pollen through a microscope. More than eight decades later Jean Perrin provided experimental evidence which verified the theoretical framework that was described by Albert Einstein three years earlier in 1905 where he related Brownian motion to the size of the particles [2]. In addition to Brownian motion, which causes the particles to undergo random translational motion, colloids also undergo significant random rotational motion due to rotational Brownian motion.

Colloids can be used as building blocks for the assembly of higher hierarchical structures. This process is referred to as colloidal self-assembly. A frequently used description of self-assembly has been provided by George Whitesides who described self-assembly as "processes that involve pre-existing components, are reversible, and can be controlled by proper design of the components" [3]. He further classified self-assembly processes as being either static or dynamic. In static self-assembly processes the system is at a local or global energy minimum and does not dissipate energy. Static self-assembly processes may require an initial input of energy but the resulting structures are stable once formed and do not require additional energy. In dynamic self-assembly processes, however, the interactions responsible for the formation of the structures are only present when the system is actively dissipating energy. Here, the system is out-of-equilibrium and the resulting structures require a continuous supply of energy as they disappear upon removing the energy supply.

Self-assembly processes require no human input. The resulting self-assembled structures result purely from the properties of the individual particles. Self-assembly thus offers a practical strategy for the formation of structures. In a typical colloidal static self-assembly process the system equilibrates to a local or global energy minimum resulting from the presence of attractive interactions between the individual colloids such as, for example, Van der Waals, magnetic or depletion attractions. Here, Brownian motion is often used as the driving force for colloidal self-assembly as these thermal fluctuations help to approach the energy minima but additional driving forces can also be used [4]. 
Note that both static and dynamic self-assembly processes are not limited to colloidal systems and may involve particles of any size and complexity. Particles larger than colloids are typically referred to as granular matter. This term generally describes particles that are roughly between $1 \mu \mathrm{m}$ and $1 \mathrm{~mm}$ in size. As a result of their relatively large size, particles in this size range typically do not display significant Brownian motion and thus different driving forces are required for their systems to reach equilibrium [5].

\subsection{Active colloids and their self-assembly processes}

Dynamic self-assembly processes require a constant input of energy to maintain their self-assembled structures as already mentioned in the previous section [6]. The supplied energy is converted into forces or motion and this dissipation of energy keeps the system out-of-equilibrium. Biological life provides many examples of active matter and dynamic self-assembly as life occurs inherently out-of-equilibrium. Here, for example, flocks of birds swarm on a macroscopic scale and motor proteins convert the chemical energy stored in ATP into mechanical work on a microscopic scale. In addition to biological systems, artificial man-made systems are also being created and studied intensively in this relatively new but rapidly evolving field of research [7-9].

In the following discussion, however, we limit ourselves to systems consisting of active colloids. Active colloids are able to convert the energy available in their environment into motion. Each of these active colloids is autonomous and able to determine its own direction of motion as they are, at least in this case, not steered or guided by external forces. In the simplest case these active colloids propel themselves in straight trajectories and only lose their directional motion on larger time scales due to rotational Brownian motion. It has been shown both experimentally and theoretically that these active colloids display the so-called motility-induced phase separation (MIPS) in the case where the active colloids show hard-sphere-like behaviour [10-13]. Here, at a sufficiently high density and activity, these active colloids cluster into a dense liquid that is surrounded by a dilute gas. MIPS can be understood by considering the collisions occurring between the active colloids. These collisions decrease the velocity of the active colloids and the influx of additional active colloids results in the formation of increasingly large clusters. Eventually, the number of incoming and outgoing active colloids equilibrates and this equilibrium determines the resulting size of the clusters. The physics of these active colloids and their MIPS has been studied extensively using computer simulations [13-15]. Note that most publications studying active colloids using computer simulations refer to them as active Brownian particles.

The introduction of additional interactions between the active colloids has been shown to result in a richer variety in the resulting phase behaviour [16]. 
For example, it has been shown both experimentally and theoretically that the introduction of dipolar interactions yields a series of novel phases [17]. Here, each hemisphere of the active colloids carried an induced charge of which both the sign and magnitude was tunable by varying the frequency of the applied alternating electric field. With the charges on both hemispheres being equal in both sign and magnitude these active colloids were purely repulsive and displayed the same behaviour as the hard-sphere-like active colloids discussed earlier. With the charges being equal in magnitude but opposite in sign these active colloids assembled into active chains due to the presence of attractive head-to-tail interactions. With the charges being unequal in both sign and magnitude these active colloids clustered when the strongest charge was present on the rear hemisphere of the active colloid. Swarming was observed when the strongest charge was present on the front hemisphere of the active colloid. Here, during swarming, the active colloids aligned themselves as a result of a torque introduced by the dipolar interactions. The induced torque present between the active colloids in this system are similar to those described in the theoretical Vicsek model [18]. In this model each particle propels itself in the average direction of its neighbouring particles plus some added noise.

Upon the addition of a short-range attraction, so that the active particles interact via a Lennard-Jones potential, the clustering of the colloids becomes reentrant [19]. Here, active colloids with a relatively low activity cluster due to the presence of attractive interactions between the particles. Upon increasing the activity of the active colloids, the active motion begins to overcome these attractive interactions which prevents the active colloids from clustering. Upon further increasing the activity, the active colloids begin to cluster again due to MIPS. While the size of the clusters resulting from MIPS is known to be difficult to control, it has been shown that it can be tuned relatively easily by the addition of weak long-range repulsive interactions [20]. The competing short-range attractive and long-range repulsive interactions limit the growth of the clusters to a certain size. To our knowledge, these models have not been verified experimentally. Besides their interactions, the properties of the individual active colloids have also been shown to influence the properties of the assembled clusters. Small clusters show different behaviour depending on the number and the orientations of the active colloids inside of the clusters. It has been shown that dimers and trimers of active colloids display either translational motion, rotational motion, both or neither [21, 22]. These clusters can possibly be used as more complex building blocks in further self-assembly processes for the development of novel phases and materials.

Furthermore, while in a relatively early stage of research, the self-assembly behaviour of active colloids with anisotropic shapes has also been studied. For active dumbbells, for example, it has been shown that the resulting clusters have a significant rotational motion [23-25]. It was found here that the introduction of a shape anisotropy decreased the activity required for MIPS to 
occur. This resulted from active colloids with anisotropic shapes also having to move in order to be able to escape the clusters whereas spherical active colloids only have to rotate. In addition, active colloidal rods have been reported to able to self-assemble into clusters with tunable properties $[26,27]$.

While most published work on active colloids focusses on these relatively simple active particles that only propel themselves in straight trajectories, active colloids with more complex motion have also been described both experimentally and theoretically. It has been shown experimentally that active colloids displaying circular motion can be created by increasing the asymmetry of the active colloids. Circling motion has been shown for granular-sized asymmetric L-shaped particles [28] and for active colloids with an asymmetric metallic patch [29]. Furthermore, by adjusting the center of mass of these active colloids, the circling motion can be directed upwards in order to obtain colloids which are self-propelled into helical trajectories [30]. In addition, accidental defects in the metal coating as a result of their synthesis methods have also been shown to result in the active colloids having active motion in circular trajectories [31]. To our knowledge, the self-assembling capabilities of these or other active colloids with complex active motion have not been reported either experimentally or theoretically.

Active colloids and their self-assembled structures are promising for, for example, in vivo drug delivery, lab-on-a-chip applications and the development of smart materials [32-34]. While these model systems have been studied extensively in the last decade, there is still a long way to go before they can be used in real world applications. The development of active colloids with an anisotropic shape and or with more complex motion and their self-assembled structures may contribute to the understanding of active systems and may be useful in one of their applications. In the following section we continue by discussing the existing experimental models for active colloids and the strategies and opportunities for the development of novel experimental models for active colloids with anisotropic shapes and more complex motion.

\subsection{Experimental model systems for active colloids}

Active colloids convert the energy available in their environment, whether chemical or physical, into motion. These active colloids have been studied extensively since their initial development in 2004 by Paxton et al. [35]. Here, bimetallic rod-shaped particles which consisted of platinum and gold ends displayed active motion in a solution of hydrogen peroxide. The breakdown of hydrogen peroxide using a bipolar redox reaction on each end of the particles surface introduces a fluid flow along the axis of the rod-shape particle which propels the particle forward [36]. This mechanism is known as self-electrophoresis. Many different designs have been reported in the litera- 
ture based on various self-propulsion mechanisms since these active colloids were first described. In the remainder of this section we briefly discuss the various preparation methods and the known self-propulsion mechanisms to power these self-propelling particles. We further discuss the opportunities for improvements on these existing designs and the development of active colloids with new designs.

All active colloids have an anisotropy in either shape or some chemical or physical property as this is a requirement for active motion to occur. The easiest and most frequently applied method to introduce this anisotropy is by partially sputter coating colloids with a thin layer of a different material in order produce chemically anisotropic colloids. These type of colloids are often referred to as Janus colloids, named after the Roman god Janus who had two faces, one on each side of his head. Depending on the material of the sputter coated layer, multiple self-propulsion mechanisms are suitable to power these colloids. The most frequently studied active colloidal model system consists of spherical colloids sputter coated with a layer of platinum which are powered by the catalytic breakdown of hydrogen peroxide on the platinum patch. The resulting pressure gradient pushes these particles forward and their velocity can be tuned by varying the concentration of hydrogen peroxide [37, 38]. This mechanism is called self-diffusiophoresis. In addition to hydrogen peroxide, other chemical fuels have also been reported in combination with suitable catalytic patches $[7,39,40]$. For example, hydrazine with an iridium patch [41] and glucose for colloids partially decorated with an enzyme that breaks down the glucose [42]. Finally, titania $\left(\mathrm{TiO}_{2}\right)$ and hematite $\left(\alpha-\mathrm{Fe}_{2} \mathrm{O}_{3}\right)$ have also been shown to be a catalyst for the breakdown of hydrogen peroxide under the exposure of ultraviolet and blue light respectively [12, 43].

In addition to chemical fuels, physical energy sources have also been used to power these active colloids. Examples of known physical fuels used for the self-propulsion of active particles include alternating electric fields [17], magnetic fields [44] and light [10,45]. Among these physical stimuli, the use of an alternating electric field has been the most frequently applied method. Here, the alternating electric field induces a charge separation in the metallic patch in this mechanism known as induced-charge electrophoresis (ICEP) [4648]. At a low frequency the ions present in the solvent are able to follow and shield these charges. Upon increasing the frequency, these ions are no longer able to follow the charges induced on the metallic patch, which results in the active colloids having a dipole moment and, as a result, the introduction of dipolar interactions between the active colloids as mentioned in the previous section. The flow of ions around the metallic patch induce an electroosmotic flow on the particles surface, which in turn results in the particle becoming self-propelled. The velocity of these active colloids can be conveniently tuned using the amplitude of the alternating electric field that is applied [49].

While using hydrogen peroxide as a fuel source to power active colloids 
is relatively easy and straightforward experimentally, this method has several downsides. Its biggest downside is the generation of oxygen bubbles which occurs predominantly when studying this model system at a high density of active colloids, therefore making self-assembly studies difficult. In addition, the concentration the of the fuel decreases during the experiment which, in turn, typically decreases the velocity of the active colloids. The use of a physical energy source eliminates these problems and these are generally more suitable mechanisms to study active colloids at a high density at the cost of having to work with a more complicated experimental setup. For further reading on the different self-propulsion mechanisms and their advantages and disadvantages we refer to references [34, 50].

These active Janus colloids are usually synthesized by first assembling the colloids into a monolayer. This allows one hemisphere of the colloids to be coated with a thin layer of metal, typically 10 to $20 \mathrm{~nm}$ thick, using sputter coating. After coating, the resulting Janus colloids can then be released from their substrate to disperse them into solution. Various methods for the preparation of colloidal monolayers exist such as, for example, drying methods, self-assembly on the air-water interface in a Langmuir-Blodgett trough, the vertical or the horizontal deposition, spin coating and dip coating [51]. Especially for active colloids driven by ICEP using alternating electric fields, it has been shown that defects present in the coating result in disturbances in the behaviour of the active colloids [34]. Thus, in order to minimize the differences observed in the behaviour of these active colloids, their colloidal monolayers should be as well-defined as possible.

A relatively new method for the preparation of colloidal monolayers is the so-called unidirectional rubbing method [52, 53]. Here, dried colloidal powders are mechanically rubbed onto a sticky surface such as, for example, polydimethylsiloxane (PDMS). Using this method well-defined two-dimensional hexagonal lattices of spherical colloids result with all colloidal crystal grains in the monolayer oriented and aligned in the direction of rubbing. The methods ease of use, its high reproducibility and its high degree of positional ordering in the resulting monolayers may make this assembly method ideal for the preparation of active Janus colloids as this should minimize the differences in behaviour between the individual active colloids. Furthermore, the alignment of the colloidal crystal grains in the the direction of rubbing allows the shape of the resulting metal coating to be controlled when sputter coated under a glancing angle [29]. Until now, the unidirectional rubbing method has only been applied for the preparation of monolayers of colloidal spheres. This method may also be suitable for the assembly of anisotropic colloids into colloidal monolayers, potentially resulting in new types of lattices.

The development novel of synthesis methods for the preparation of anisotropic colloids may allow the preparation of active colloids with different and possibly more complex behaviour in the future. Examples of developments 
in colloidal synthesis methods for the preparation of anisotropic colloids are polystyrene dimers and trimers [54] and hematite cubes [55]. Anisotropic particles may not only display a more complex active motion, but may also form more complex dynamically self-assembled structures. Furthermore, developments in colloidal synthesis methods potentially also allow for the preparation of active colloids in much larger yields. The yields of synthesis methods in which colloidal monolayers are sputter coated with a thin layer of metal are inherently small. Synthesis methods where the active colloids are prepared in a bulk liquid, on the other hand, generally have much higher yields. Examples of these bulk methods include the encapsulation of colloidal hematite cubes into oil droplets and subsequently solidifying this oil encapsulation [56] and the assembly into pickering emulsions where the colloids are partially coated on the liquid-liquid interface $[57,58]$. For large scale applications of active colloids the use of bulk syntheses methods may be required.

\subsection{Colloidal silica superballs and their monolayers}

An example of colloids with an anisotropic shape are the so-called colloidal superballs. The superball shape is an interpolation between the spherical and the cubic shape. The contours of the superball shape are defined by the following formula $[59,60]$ :

$$
\left|\frac{2 x}{D}\right|^{m}+\left|\frac{2 y}{D}\right|^{m}+\left|\frac{2 z}{D}\right|^{m}=1,
$$

where $m$ is the so-called shape parameter and $D$ is the edge diameter (faceto-face length) of the superballs and $x, y$ and $z$ are the coordinates of the surface along the $x$-, $y$ - and $z$-axes respectively. The shape parameter $m$ ranges from $m=2$ for a sphere to $m \rightarrow \infty$ for a cube. Examples of superball particles with various shapes ranging from spherical to cubic along with their corresponding shape parameter are schematically depicted in figure 1.1. A synthesis route for the preparation of colloids with the superball shape is based on the synthesis of colloidal hematite cubes, which was developed and published by Tatao Sugimoto and Kazuo Sakata in 1992 [55]. By coating these colloidal hematite cubes with a layer of silica and subsequently dissolving the magnetic core particle, hollow silica superballs can be prepared. The complete synthesis route was first described by Laura Rossi et al. in 2011 [61]. The synthesis of these colloidal superballs can be tuned in order to vary the shape parameter of the resulting superballs. Note here that using this synthesis route only superballs with a shape parameter of up to approximately $m=4$ can be prepared due to the experimental limitations of the colloidal synthesis with which the colloidal hematite cubes are prepared [62]. In figure 1.1 it can be seen, however, that superballs with a shape parameter of $m=4$ already have 
a significant cube-like shape so that a relatively wide range of superball shapes can still be prepared using this synthesis route.

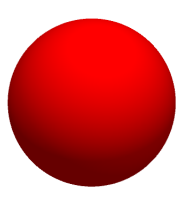

$$
m=2
$$

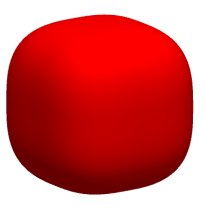

$m=3$

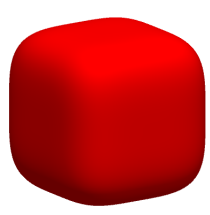

$m=4$

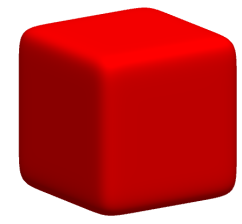

$m=10$

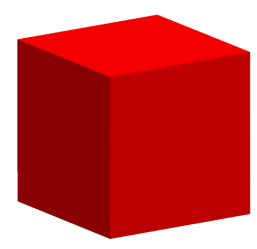

$m \rightarrow \infty$

Figure 1.1: Schematic illustration of superball particles with various shape parameters ranging from $m=2$ for spherical particles to $m \rightarrow \infty$ for cubic particles.

The variable shape of these colloidal superballs makes them excellent candidates for studying the influence of shape on the phase behaviour and phase transitions. Since their development, the phase behaviour of these colloidal superballs has been studied in detail using various assembly methods in both two and three dimensions [63-65]. In these studies the resulting structures were not only observed to result from the variable superball shape, but were also found to be dependent on the assembly method and the interactions present between the individual colloidal superballs. In the following discussion, however, we limit ourselves to the assembly of these colloidal superballs in two dimensions. In two dimensions, colloidal superballs have been observed to be able to assemble into several different phases. The three types of lattices these colloidal superballs are known to assemble in are the square-, the $\Lambda_{0^{-}}$and the $\Lambda_{1}$-lattice. These lattices are schematically depicted in figure 1.2. The $\Lambda_{0^{-}}$ and the $\Lambda_{1}$-lattices are more densely packed than the square lattice for all values of the shape parameter $m$. Using molecular dynamics simulations it has been shown that at a shape parameter of approximately $m=2.6$ the $\Lambda_{0^{-}}$ and the $\Lambda_{1}$-lattices have an equally dense packing. The $\Lambda_{0}$-lattice has a denser packing below this value and the $\Lambda_{1}$-lattice has a denser packing above this value [59]. The difference between the packing densities of the $\Lambda_{0^{-}}$and the $\Lambda_{1}$-lattices, however, are small and only differ about $1 \%$ from each other at a shape parameter of $m=4$.

The assembly of these colloidal superballs into well-defined lattices has been demonstrated using both the horizontal and vertical deposition method by Janne-Mieke Meijer et al. [60, 62]. In both methods the colloidal superballs assemble at the evaporating contact line with the main difference between the two methods being the orientation of the substrate. Here, the strong immersion capillary forces present between the particles compresses them into densely packed lattices. For all of the differently shaped superballs tested within the range of $2.9<m<3.6$ a mixture of both the $\Lambda_{0^{-}}$and $\Lambda_{1}$-lattices was observed with only a minor fraction of the particles being packed in the less dense cubic-lattice structure. Upon increasing the shape parameter of 


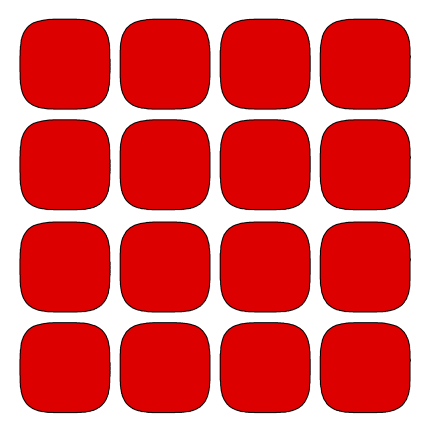

Cubic-lattice

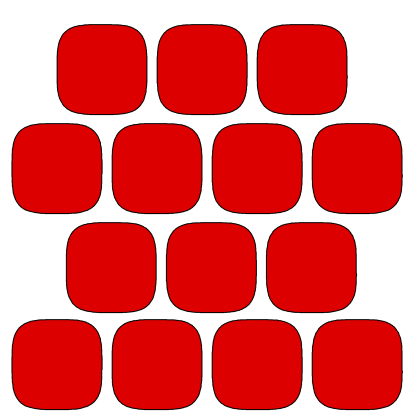

$\Lambda_{0}$-lattice

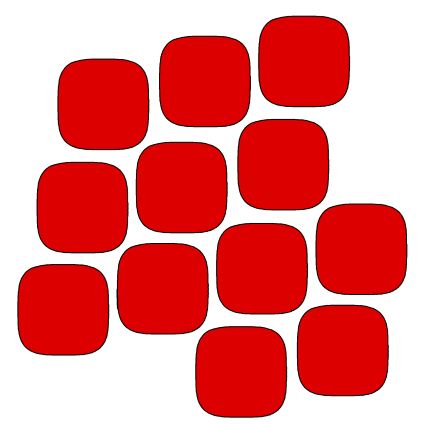

$\Lambda_{1}$-lattice

Figure 1.2: Schematic representation of the two-dimensional lattices colloidal superballs are known to be able to assemble into. These are the cubic-, the $\Lambda_{0^{-}}$and the $\Lambda_{1}$-lattices. The shape parameter $m$ of the colloidal superballs is $m=4$ in each of the representations.

the superballs, however, the ratio of the fraction of the particles being packed into the $\Lambda_{1}$-lattice to the $\Lambda_{0}$-lattice was observed to increase as a result of the $\Lambda_{1}$-lattice becoming increasingly more dense upon an increase in the shape parameter. The phase behaviour observed in these experiments agree with those found in Monte Carlo simulations [66]. The horizontal and vertical deposition method allows the formation of relatively large and cm-sized lattices.

Finally, the self-assembly of colloidal superballs into well-defined lattices has been shown by Laura Rossi et al. using depletion interaction [61, 67]. Here, the resulting phases were found to depend on both the shape parameter of the superballs and the radius of gyration of the depletant relative to the size of the superballs. The $\Lambda_{0}$-lattice was observed to form for all superball shapes with a shape parameter below approximately $m=2.7$. Above this shape parameter value, the more cube-like colloidal superballs formed either the square-lattice using depletants with a relatively small radius of gyration or the $\Lambda_{1}$-lattice using depletants with a relatively larger radius of gyration. The transition between these lattices results from the larger depletants not fitting into the pores of the cubic-lattice. In this example the self-assembly is static and the resulting lattices are at equilibrium.

\subsection{Outline of this thesis}

This thesis focusses on the development of novel pathways for the preparation of active colloids. In addition, we study the lattices of colloidal superball monolayers assembled using the unidirectional rubbing method in detail. This thesis does not only serve to present the results that we have obtained, but also as a guide for those who want to continue with this research.

In Chapter 2 we describe the most important experimental methods used 
throughout this thesis. We first describe the synthesis methods for the preparation of the colloidal silica superballs and the colloidal polymer spheres. The latter were used to prepare the active colloids shown later in this thesis. We then present a custom-made device used for the assembly of the silica superballs into colloidal monolayers using the unidirectional rubbing method. Finally we describe the custom-made cells that were used to study the active colloids under the influence of an alternating electric field in situ using optical microscopy.

In Chapter 3 we present a strategy for the small-angle X-ray scattering (SAXS) analysis of colloidal silica superball monolayers assembled using the unidirectional rubbing method. Using three types of colloidal superballs with shapes ranging from spherical to almost cubic we illustrate the analysis of the resulting diffraction patterns imaged in reciprocal space and provide a model for the superball lattices in real space. In addition, we describe the influence of the form factor of the superball particles which complicates the analysis of the diffraction patterns.

In Chapter 4 we continue by studying the influence of the size and shape of the colloidal superballs on the resulting monolayer lattices. Furthermore, we study the influence of the rubbing speed and pressure at which these colloidal superball monolayers are prepared. In addition to SAXS, we employ scanning electron microscopy (SEM) to study the colloidal superball lattices in real space. We end this part of the thesis by providing an explanation for the observed superball lattices.

In Chapter 5 we describe the so-called active colloidal circlers and spinners. These active colloids become self-propelled in alternating electric fields. We show how the design of the active colloidal circlers affects the observed circular motion of the particles and describe the active properties of these effectively chiral active colloids. In addition, we show how both the magnitude and frequency of the alternating electric field influences the behaviour of the active colloidal circlers. Finally, we describe the behaviour of the active colloidal spinners. Using a modification in the design of these active colloids, the translational active motion can be virtually eliminated so that these active colloids only rotate in place.

In Chapter 6, the final chapter of this thesis, we present a novel synthesis method for the preparation of active anisotropic hydrogel particles. These particles are prepared by locally polymerizing a spin coated layer of PEGDA on hydrophilized glass slides using ultraviolet (UV) light. We show how the size and shape of the resulting particles can be controlled and adjusted. This method relies only on inexpensive materials and only requires a motorized microscope equipped with an UV light source and allows the production of about 10.000 particles per hour. Finally, we briefly show the active motion of these active anisotropic hydrogel particles observed under the influence of an alternating electric field. 


\section{References}

[1] S. G. Mokrushin, "Thomas Graham and the definition of colloids," Nature, no. 4844, p. 861, 1962.

[2] R. Newburgh, J. Peidle, and W. Rueckner, "Einstein, Perrin, and the reality of atoms: 1905 revisited," American Journal of Physics, vol. 74, no. 6, pp. 478481, 2006.

[3] W. George and G. Bartosz, "Self-Assembly at All Scales," Science, vol. 295, no. March, pp. 2418-2421, 2002.

[4] F. Li, D. P. Josephson, and A. Stein, "Colloidal assembly: The road from particles to colloidal molecules and crystals," Angewandte Chemie - International Edition, vol. 50, no. 2, pp. 360-388, 2011.

[5] R. Groß and M. Dorigo, "Self-assembly at the macroscopic scale," Proceedings of the IEEE, vol. 96, no. 9, pp. 1490-1508, 2008.

[6] G. M. Whitesides and M. Boncheva, "Supramolecular chemistry and self-assembly special feature: Beyond molecules: Self-assembly of mesoscopic and macroscopic components," Proceedings of the National Academy of Sciences of the United States of America, vol. 99, no. 8, p. 4769, 2002.

[7] A. M. Menzel, "Tuned, driven, and active soft matter," Physics Reports, vol. 554, pp. 1-45, 2015.

[8] M. Das, C. F. Schmidt, and M. Murrell, "Introduction to active matter," Soft Matter, vol. 16, no. 31, pp. 7185-7190, 2020.

[9] G. Gompper, R. G. Winkler, T. Speck, A. Solon, C. Nardini, F. Peruani, H. Löwen, R. Golestanian, U. B. Kaupp, L. Alvarez, T. Ki rboe, E. Lauga, W. C. Poon, A. Desimone, S. Muiños-Landin, A. Fischer, N. A. Söker, F. Cichos, R. Kapral, P. Gaspard, M. Ripoll, F. Sagues, A. Doostmohammadi, J. M. Yeomans, I. S. Aranson, C. Bechinger, H. Stark, C. K. Hemelrijk, F. o. J. Nedelec, T. Sarkar, T. Aryaksama, M. Lacroix, G. Duclos, V. Yashunsky, P. Silberzan, M. Arroyo, and S. Kale, "The 2020 motile active matter roadmap," Journal of Physics Condensed Matter, vol. 32, no. 19, 2020.

[10] I. Buttinoni, J. Bialké, F. Kümmel, H. Löwen, C. Bechinger, and T. Speck, "Dynamical clustering and phase separation in suspensions of self-propelled colloidal particles," Physical Review Letters, vol. 110, no. 23, pp. 1-5, 2013.

[11] J. Bialké, T. Speck, and H. Löwen, "Active colloidal suspensions: Clustering and phase behavior," Journal of Non-Crystalline Solids, vol. 407, pp. 367-375, 2015 .

[12] J. Palacci, S. Sacanna, A. P. Steinberg, D. J. Pine, and P. M. Chaikin, "Living crystals of light-activated colloidal surfers," Science, vol. 339, no. 6122, pp. 936940, 2013.

[13] G. Gonnella, D. Marenduzzo, A. Suma, and A. Tiribocchi, "Motility-induced phase separation and coarsening in active matter," Comptes Rendus Physique, vol. 16, no. 3, pp. 316-331, 2015. 
[14] P. Romanczuk, M. Bär, W. Ebeling, B. Lindner, and L. Schimansky-Geier, "Active Brownian particles: From individual to collective stochastic dynamics: From individual to collective stochastic dynamics," European Physical Journal: Special Topics, vol. 202, no. 1, pp. 1-162, 2012.

[15] M. E. Cates and J. Tailleur, "Motility-induced phase separation," Annual Review of Condensed Matter Physics, vol. 6, no. 1, pp. 219-244, 2015.

[16] S. A. Mallory, C. Valeriani, and A. Cacciuto, "An active approach to colloidal self-assembly," Annual Review of Physical Chemistry, vol. 69, pp. 59-79, 2018.

[17] J. Yan, M. Han, J. Zhang, C. Xu, E. Luijten, and S. Granick, "Reconfiguring active particles by electrostatic imbalance," Nature Materials, vol. 15, no. 10, pp. 1095-1099, 2016.

[18] T. Vicsek, A. Czirók, E. Ben-Jacob, I. Cohen, and O. Shochet, "Novel type of phase transition in a system of self-driven particles," Phys. Rev. Lett., vol. 75, no. 6, pp. 1226-1229, Aug. 1995.

[19] G. S. Redner, A. Baskaran, and M. F. Hagan, "Reentrant phase behavior in active colloids with attraction," Physical Review E-Statistical, Nonlinear, and Soft Matter Physics, vol. 88, no. 1, pp. 1-5, 2013.

[20] E. Mani and H. Löwen, "Effect of self-propulsion on equilibrium clustering," Physical Review E - Statistical, Nonlinear, and Soft Matter Physics, vol. 92, no. 3, pp. 1-7, 2015.

[21] W. Gao, A. Pei, X. Feng, C. Hennessy, and J. Wang, "Organized self-assembly of janus micromotors with hydrophobic hemispheres," Journal of the American Chemical Society, vol. 135, no. 3, pp. 998-1001, 2013.

[22] Q. Zhang, R. Dong, X. Chang, B. Ren, and Z. Tong, "Spiropyran-decorated SiO2-Pt JanusmMicromotor: Preparation and light-induced dynamic self-assembly and disassembly," ACS Applied Materials and Interfaces, vol. 7, no. 44, pp. 24585$24591,2015$.

[23] A. Suma, G. Gonnella, D. Marenduzzo, and E. Orlandini, "Motility-induced phase separation in an active dumbbell fluid," Epl, vol. 108, no. 5, 2014.

[24] C. Tung, J. Harder, C. Valeriani, and A. Cacciuto, "Micro-phase separation in two dimensional suspensions of self-propelled spheres and dumbbells," Soft Matter, vol. 12, no. 2, pp. 555-561, 2015.

[25] P. Digregorio, D. Levis, A. Suma, L. F. Cugliandolo, G. Gonnella, and I. Pagonabarraga, "2D melting and motility induced phase separation in Active Brownian Hard Disks and Dumbbells," Journal of Physics: Conference Series, vol. 1163, no. 1, 2019.

[26] M. S. Davies Wykes, J. Palacci, T. Adachi, L. Ristroph, X. Zhong, M. D. Ward, J. Zhang, and M. J. Shelley, "Dynamic self-assembly of microscale rotors and swimmers," Soft Matter, vol. 12, no. 20, pp. 4584-4589, 2016.

[27] Y. Wang, S. T. Fei, Y. M. Byun, P. E. Lammert, V. H. Crespi, A. Sen, and T. E. Mallouk, "Dynamic interactions between fast microscale rotors," Journal of the American Chemical Society, vol. 131, no. 29, pp. 9926-9927, 2009. 
[28] F. Kümmel, B. Ten Hagen, R. Wittkowski, I. Buttinoni, R. Eichhorn, G. Volpe, H. Löwen, and C. Bechinger, "Circular motion of asymmetric self-propelling particles," Physical Review Letters, vol. 110, no. 19, pp. 1-5, 2013.

[29] R. J. Archer, A. I. Campbell, and S. J. Ebbens, "Glancing angle metal evaporation synthesis of catalytic swimming Janus colloids with well defined angular velocity," Soft Matter, vol. 11, no. 34, pp. 6872-6880, 2015.

[30] A. I. Campbell, R. Wittkowski, B. Ten Hagen, H. Löwen, and S. J. Ebbens, "Helical paths, gravitaxis, and separation phenomena for mass-anisotropic selfpropelling colloids: Experiment versus theory," Journal of Chemical Physics, vol. 147 , no. $8,2017$.

[31] X. Wang, M. In, C. Blanc, A. Würger, M. Nobili, and A. Stocco, "Janus colloids actively rotating on the surface of water," Langmuir, vol. 33, no. 48, pp. 13766 $13773,2017$.

[32] S. J. Ebbens and J. R. Howse, "In pursuit of propulsion at the nanoscale," Soft Matter, vol. 6, no. 4, pp. 726-738, 2010.

[33] S. J. Ebbens, "Active colloids: Progress and challenges towards realising autonomous applications," Current Opinion in Colloid and Interface Science, vol. 21, pp. 14-23, 2016.

[34] W. Wang, X. Lv, J. L. Moran, S. Duan, and C. Zhou, "A practical guide to active colloids: choosing synthetic model systems for soft matter physics research," Soft Matter, vol. 16, no. 16, pp. 3846-3868, 2020.

[35] W. F. Paxton, K. C. Kistler, C. C. Olmeda, A. Sen, S. K. St. Angelo, Y. Cao, T. E. Mallouk, P. E. Lammert, and V. H. Crespi, "Catalytic nanomotors: Autonomous movement of striped nanorods," Journal of the American Chemical Society, vol. 126, no. 41, pp. 13424-13431, 2004.

[36] W. F. Paxton, P. T. Baker, T. R. Kline, Y. Wang, T. E. Mallouk, and A. Sen, "Catalytically induced electrokinetics for motors and micropumps," Journal of the American Chemical Society, vol. 128, no. 46, pp. 14881-14888, 2006.

[37] J. R. Howse, R. A. Jones, A. J. Ryan, T. Gough, R. Vafabakhsh, and R. Golestanian, "Self-motile colloidal particles: From directed propulsion to random walk," Physical Review Letters, vol. 99, no. 4, pp. 8-11, 2007.

[38] Y. Shelke, N. R. Srinivasan, S. P. Thampi, and E. Mani, "Transition from linear to circular motion in active spherical-cap colloids," Langmuir, vol. 35, no. 13, pp. 4718-4725, 2019.

[39] J. Zhang, E. Luijten, B. A. Grzybowski, and S. Granick, "Active colloids with collective mobility status and research opportunities," Chemical Society Reviews, vol. 46, no. 18, pp. 5551-5569, 2017.

[40] J. Zhang, B. A. Grzybowski, and S. Granick, "Janus particle synthesis, assembly, and application," Langmuir, vol. 33, no. 28, pp. 6964-6977, 2017.

[41] W. Gao, A. Pei, R. Dong, and J. Wang, "Catalytic iridium-based Janus micromotors powered by ultralow levels of chemical fuels," Journal of the American Chemical Society, vol. 136, no. 6, pp. 2276-2279, 2014. 
[42] P. Schattling, B. Thingholm, and B. Städler, "Enhanced diffusion of glucosefueled Janus particles," Chemistry of Materials, vol. 27, no. 21, pp. 7412-7418, 2015.

[43] J. Palacci, S. Sacanna, S. Kim, G. Yi, D. J. Pine, and P. M. Chaikin, "Colloids," 2014.

[44] J. Yan, S. C. Bae, and S. Granick, "Rotating crystals of magnetic Janus colloids," Soft Matter, vol. 11, no. 1, pp. 147-153, 2015.

[45] D. P. Singh, U. Choudhury, P. Fischer, and A. G. Mark, "Non-equilibrium assembly of light-activated colloidal mixtures," Advanced Materials, vol. 29, no. 32, pp. 1-7, 2017.

[46] E. Yariv, "Induced-charge electrophoresis of nonspherical particles," Physics of Fluids, vol. 17, no. 5, pp. 1-4, 2005.

[47] S. Gangwal, O. J. Cayre, and O. D. Velev, "Dielectrophoretic assembly of metallodielectric janus particles in AC electric fields," Langmuir, vol. 24, no. 23, pp. 13312-13 320, 2008.

[48] M. Z. Bazant, M. S. Kilic, B. D. Storey, and A. Ajdari, "Towards an understanding of induced-charge electrokinetics at large applied voltages in concentrated solutions," Advances in Colloid and Interface Science, vol. 152, no. 1-2, pp. 4888, 2009.

[49] S. Gangwal, O. J. Cayre, M. Z. Bazant, and O. D. Velev, "Induced-charge electrophoresis of metallodielectric particles," Physical Review Letters, vol. 100, no. 5, pp. 1-4, 2008.

[50] C. W. Shields and O. D. Velev, "The evolution of active particles: Toward externally powered self-propelling and self-reconfiguring particle systems," Chem, vol. 3, no. 4, pp. 539-559, 2017.

[51] Z. Xu, L. Wang, F. Fang, Y. Fu, and Z. Yin, "A review on colloidal self-assembly and their applications," Current Nanoscience, vol. 12, no. 6, pp. 725-746, 2016.

[52] C. Park, T. Lee, Y. Xia, T. J. Shin, J. Myoung, and U. Jeong, "Quick, large-area assembly of a single-crystal monolayer of spherical particles by unidirectional rubbing," Advanced Materials, vol. 26, no. 27, pp. 4633-4638, 2014.

[53] C. Park, K. Koh, and U. Jeong, "Structural Color Painting by Rubbing Particle Powder," Scientific Reports, vol. 5, pp. 1-5, 2015.

[54] J. R. Wolters, G. Avvisati, F. Hagemans, T. Vissers, D. J. Kraft, M. Dijkstra, and W. K. Kegel, "Self-assembly of "mickey Mouse" shaped colloids into tube-like structures: Experiments and simulations," Soft Matter, vol. 11, no. 6, pp. 1067-1077, 2015.

[55] T. Sugimoto and T. Sakata, "Preparation of monodisperse pseudocubic $\alpha$ Fe2O3 particles from condensed ferric hydroxide gel," Journal of Colloid And Interface Science, vol. 152, no. 2, pp. 587-590, 1992.

[56] J. Palacci, S. Sacanna, A. Abramian, J. Barral, K. Hanson, A. Y. Grosberg, D. J. Pine, and P. M. Chaikin, "Artificial rheotaxis," Science Advances, vol. 1, no. 4, pp. 1-6, 2015. 
[57] L. Hong, S. Jiang, and S. Granick, "Simple method to produce janus colloidal particles in large quantity," Langmuir, vol. 22, no. 23, pp. 9495-9499, 2006.

[58] R. J. Archer, A. J. Parnell, A. I. Campbell, J. R. Howse, and S. J. Ebbens, "A pickering emulsion foute to swimming active Janus colloids," Advanced Science, vol. 5, no. 2, pp. 1-9, 2018.

[59] Y. Jiao, F. H. Stillinger, and S. Torquato, "Optimal packings of superdisks and the role of symmetry," Physical Review Letters, vol. 100, no. 24, pp. 2-5, 2008.

[60] J. M. Meijer, F. Hagemans, L. Rossi, D. V. Byelov, S. I. Castillo, A. Snigirev, I. Snigireva, A. P. Philipse, and A. V. Petukhov, "Self-assembly of colloidal cubes via vertical deposition," Langmuir, vol. 28, no. 20, pp. 7631-7638, 2012.

[61] L. Rossi, S. Sacanna, W. T. Irvine, P. M. Chaikin, D. J. Pine, and A. P. Philipse, "Cubic crystals from cubic colloids," Soft Matter, vol. 7, no. 9, pp. 4139-4142, 2011.

[62] J. M. Meijer, V. Meester, F. Hagemans, H. N. Lekkerkerker, A. P. Philipse, and A. V. Petukhov, "Convectively assembled monolayers of colloidal cubes: Evidence of optimal packings," Langmuir, vol. 35, no. 14, pp. 4946-4955, 2019.

[63] J. M. Meijer, D. V. Byelov, L. Rossi, A. Snigirev, I. Snigireva, A. P. Philipse, and A. V. Petukhov, "Self-assembly of colloidal hematite cubes: A microradian Xray diffraction exploration of sedimentary crystals," Soft Matter, vol. 9, no. 45, pp. $10729-10738,2013$.

[64] J. M. Meijer, A. Shabalin, R. Dronyak, O. M. Yefanov, A. Singer, R. P. Kurta, U. Lorenz, O. Gorobstov, D. Dzhigaev, J. Gulden, D. V. Byelov, A. V. Zozulya, M. Sprung, I. A. Vartanyants, and A. V. Petukhov, "Double hexagonal close-packed structure revealed in a single colloidal crystal grain by Bragg rod analysis," Journal of Applied Crystallography, vol. 47, no. 4, pp. 1199-1204, 2014.

[65] J. M. Meijer, A. Pal, S. Ouhajji, H. N. Lekkerkerker, A. P. Philipse, and A. V. Petukhov, "Observation of solid-solid transitions in 3D crystals of colloidal superballs," Nature Communications, vol. 8, pp. 1-8, 2017.

[66] Z. L. Hou, Y. Ju, Y. W. Zong, F. F. Ye, and K. Zhao, "Molecular dynamics simulations on the dynamics of two-dimensional rounded squares," Chinese Physics B, vol. 27, no. 8, 2018.

[67] L. Rossi, V. Soni, D. J. Ashton, D. J. Pine, A. P. Philipse, P. M. Chaikin, M. Dijkstra, S. Sacanna, and W. T. Irvine, "Shape-sensitive crystallization in colloidal superball fluids," Proceedings of the National Academy of Sciences of the United States of America, vol. 112, no. 17, pp. 5286-5290, 2015. 


\title{
Chapter 2
}

\section{General Experimental Methods}

\begin{abstract}
In this chapter we describe the most important experimental techniques used throughout this thesis. We first show the synthesis methods to produce colloidal silica superballs with a tunable shape and size and their characterization. We then present the device we have designed for the assembly of colloids into monolayers. This open-source device allows colloidal monolayers to be prepared using the unidirectional rubbing method in a tunable and reproducible fashion. Finally, we show the custom-made cells which allows colloidal dispersions to be studied under the influence of an alternating electric field in situ using optical microscopy. These experimental techniques are described in detail for this work to be continued and expanded upon in the future.
\end{abstract}




\subsection{Synthesis and characterization of colloidal silica superballs}

\subsubsection{Synthesis of colloidal silica spheres}

Colloidal silica spheres were synthesized using the so-called Stöber process which was first described by Werner Stöber and Arthur Fink in 1968 [1]. This well-known synthesis method has been widely used and studied and many variants of this synthesis method have been developed since [2, 3]. Most Stöber-based synthesis methods focus on the preparation of colloidal silica spheres with a diameter of approximately $1 \mu \mathrm{m}$ or smaller. The preparation of colloidal silica spheres with a significantly larger diameter and with a relatively low polydispersity of around and below $5 \%$, however, is often troublesome.

Using the following recipe we have prepared colloidal silica spheres with diameters of up to around $1.5 \mu \mathrm{m}$ and with polydispersities of around $5 \%$. Here, a mixture of $2 \mathrm{~mL}$ tetraethyl orthosilicate (TEOS, Sigma Aldrich, $\geq$ $99 \%$ ) in $8 \mathrm{~mL}$ ethanol (Emsure, ACS ISO reagent) was quickly added to a mixture of $11.6 \mathrm{~mL}$ ammonia (Acros, 28-30 wt.\% in water, newly opened) in $40.4 \mathrm{~mL}$ ethanol in a $250 \mathrm{~mL}$ round-bottom flask under vigorous magnetic stirring. This reaction was allowed to proceed for 2 hours at room temperature after which a small volume was removed in order to analyse the intermediate size of the particles. At this point the colloidal silica spheres were observed to have diameters of around $D=0.7 \mu \mathrm{m}$ with polydispersities of around $\sigma_{D}=$ $10 \%$. Now a second portion of TEOS in ethanol (1:4 in volume) was added to the flask to further increase the size of the colloidal silica spheres. The final size of the colloidal silica spheres was tuned by the volume of the second portion of the TEOS and ethanol mixture that was added to the reaction. Here, the volume of TEOS was adjusted from 7 to $20 \mathrm{~mL}$ in order to obtain colloidal silica spheres with a final a diameter of $D=1.0$ to $1.5 \mu \mathrm{m}$ respectively and with a polydispersity of around $\sigma_{D}=5 \%$. After allowing the reaction to proceed for another 2 hours the resulting colloidal silica spheres were washed with ethanol three times using centrifugation.

To obtain the colloidal silica spheres reproducibly and with a low polydispersity it is important to use clean glassware for the reaction as silica is known to nucleate easily on surfaces. In addition, care should be taken not to use ammonia from a bottle that has been opened and used too frequently as the concentration of the ammonia solution decreases every time the bottle is opened. While the resulting colloidal silica spheres may still be usable, their resulting sizes can be difficult to predict

\subsubsection{Synthesis of non-spherical colloidal silica superballs}

Non-spherical colloidal superballs were synthesized by coating colloidal hematite cubes, which were first reported by Tadao Sugimoto and Kazuo Sakata 
and published in 1992 [4], with a layer of silica and subsequently dissolving the magnetic core particle. This complete synthesis method was first described by Rossi et al. in 2011 [5].

Colloidal hematite cubes were synthesized by first dissolving an amount of sodium hydroxide (Emsure, pellets) in approximately $80 \mathrm{~mL}$ of water (Milli-Q, $18.2 \mathrm{M} \Omega \cdot \mathrm{cm}$ ) in a $100 \mathrm{~mL}$ volumetric flask. After the sodium hydroxide was completely dissolved and the solution had cooled down to room temperature the volumetric flask was filled with water to $100 \mathrm{~mL}$. Next, $50.0 \mathrm{~g}$ of iron(III) chloride hexahydrate (Sigma-Aldrich) was quickly weighed in a $250 \mathrm{~mL}$ Pyrex bottle to which $100 \mathrm{~mL}$ of water was added. The bottle was ultrasonicated for 20 minutes in order to dissolve the iron(III) chloride. The sodium hydroxide solution was then added to the Pyrex bottle over the course of approximately 18 seconds using a funnel under vigorous magnetic stirring using a very large magnetic stirring bar. Stirring was continued for 10 minutes after the addition of the sodium hydroxide solution was completed, after which the bottle was transferred to a pre-heated oven set at a temperature of $100{ }^{\circ} \mathrm{C}$ for a period of 8 days. After this time the bottles were left to cool down to room temperature and the resulting colloidal hematite cubes were washed six times with water and three times with ethanol (Emsure, ACS ISO reagent) using centrifugation.

The size of the resulting colloidal hematite cubes was tuned by the amount of sodium hydroxide used in the first step of the reaction. The amount of sodium hydroxide added determines the excess concentration of ferric ions which, in turn, controls the size of the resulting hematite cubes [6]. Here, the amount of sodium hydroxide was varied between 19.2 and $19.6 \mathrm{~g}$ to obtain colloidal hematite cubes with edge diameters between roughly $D=0.7$ and $1.5 \mu \mathrm{m}$. Care should be taken during this synthesis as the resulting size of the colloidal hematite cubes is known to be very sensitive on the concentration of both the sodium hydroxide and the iron(III) chloride solution and the time in which the former solution is added to the latter solution [4, 6]. Previously unopened iron(III) chloride containers should be used for this synthesis due to its high hygroscopicity.

The colloidal hematite cubes were then coated with a layer of silica. First, around $1 \mathrm{~g}$ of the colloidal hematite cubes dispersed in $20 \mathrm{~mL}$ water was mixed with $150 \mathrm{~mL}$ of an aqueous $35 \mathrm{~g} / \mathrm{L}$ polyvinylpyrrolidone $\left(\mathrm{PVP}, M_{w}=\right.$ $40 \mathrm{~kg} / \mathrm{mol}$, Sigma-Aldrich) solution that was then stirred overnight. The nonadsorbed PVP was removed the following day by washing the colloidal hematite cubes with ethanol three times to a final volume of $20 \mathrm{~mL}$. A $1 \mathrm{~L}$ roundbottom flask was then filled with $380 \mathrm{~mL}$ ethanol, $66 \mathrm{~mL}$ water, $10 \mathrm{~mL}$ of an aqueous 1 wt.\% tetramethylammonium hydroxide (TMAH, Sigma-Aldrich) solution and the colloidal hematite cubes dispersed in $20 \mathrm{~mL}$ ethanol under mechanical stirring and ultrasonication at room temperature. A mixture of $10 \mathrm{~mL}$ 
tetraethyl orthosilicate (TEOS, Sigma-Aldrich, $\geq 99 \%$ ) in $10 \mathrm{~mL}$ ethanol was then added using a peristaltic pump at a rate of $0.4 \mathrm{~mL} / \mathrm{min}$. Ultrasonication was continued for at least 2 hours after the addition of the TEOS mixture was completed and the mechanical stirring was continued overnight. The now with silica coated colloidal hematite cubes were washed three times with ethanol using centrifugation the following day.

Finally, the hematite core of the silica coated colloidal hematite cubes were dissolved using hydrochloric acid by mixing an aqueous dispersion of the silica coated colloidal hematite cubes with hydrochloric acid to a final concentration of $6 \mathrm{M}$ and leaving the vials on a roller mixer until their color had become white. This process typically took several days.

In order to synthesize colloidal silica superballs with relatively low shape parameter, approximately between $m=2.5$ and 3 , additional layers of silica were grown on the silica coated colloidal hematite cubes. These additional layers were grown using the same recipe as the first layer with the silica coated colloidal hematite cubes replacing the colloidal hematite cubes which are dispersed in the $20 \mathrm{~mL}$ of ethanol. The hematite cores were only removed after all layers of silica were grown in order to separate the colloidal silica superballs from the secondary nucleates more easily. With each layer of silica, the thickness of the silica shell increases and its shape parameter $m$ decreases. Up to four silica coatings were required to synthesize colloidal silica superballs with a shape parameter of approximately $m=2.5$. Note here that the resulting hollow colloidal silica superballs, especially those with thin shells, should not be stirred magnetically for extended periods of time as the stirrer bar will crush the shells. These silica colloids should also not be dispersed in water for extended periods of time as silica is known to slowly dissolve in water.

\subsubsection{Characterization of colloidal silica superballs}

The average shape and size of the synthesized colloidal silica superballs were determined using transmission electron microscopy (TEM) with a Tecnai 10 electron microscope. Representative TEM images of some examples of these superballs with diameters of approximately $D=1 \mu \mathrm{m}$ and various shapes ranging from spherical to almost cubic are depicted in figure 2.1. The shape parameters are $m=2,2.87,3.32$ and 3.63 for the superballs depicted in figure $2.1 \mathrm{a}, \mathrm{b}, \mathrm{c}$ and $\mathrm{d}$ respectively. The hollow nature of the superballs is visible in the TEM images for superballs with a relatively high shape parameter as more electrons are able to pass through these particles as indicated by the lighter inner colour in the TEM images. As a result of the synthesis method, the superballs with a relatively high shape parameter have relatively thin shells while the superballs with lower shape parameter have relatively thicker shells. 

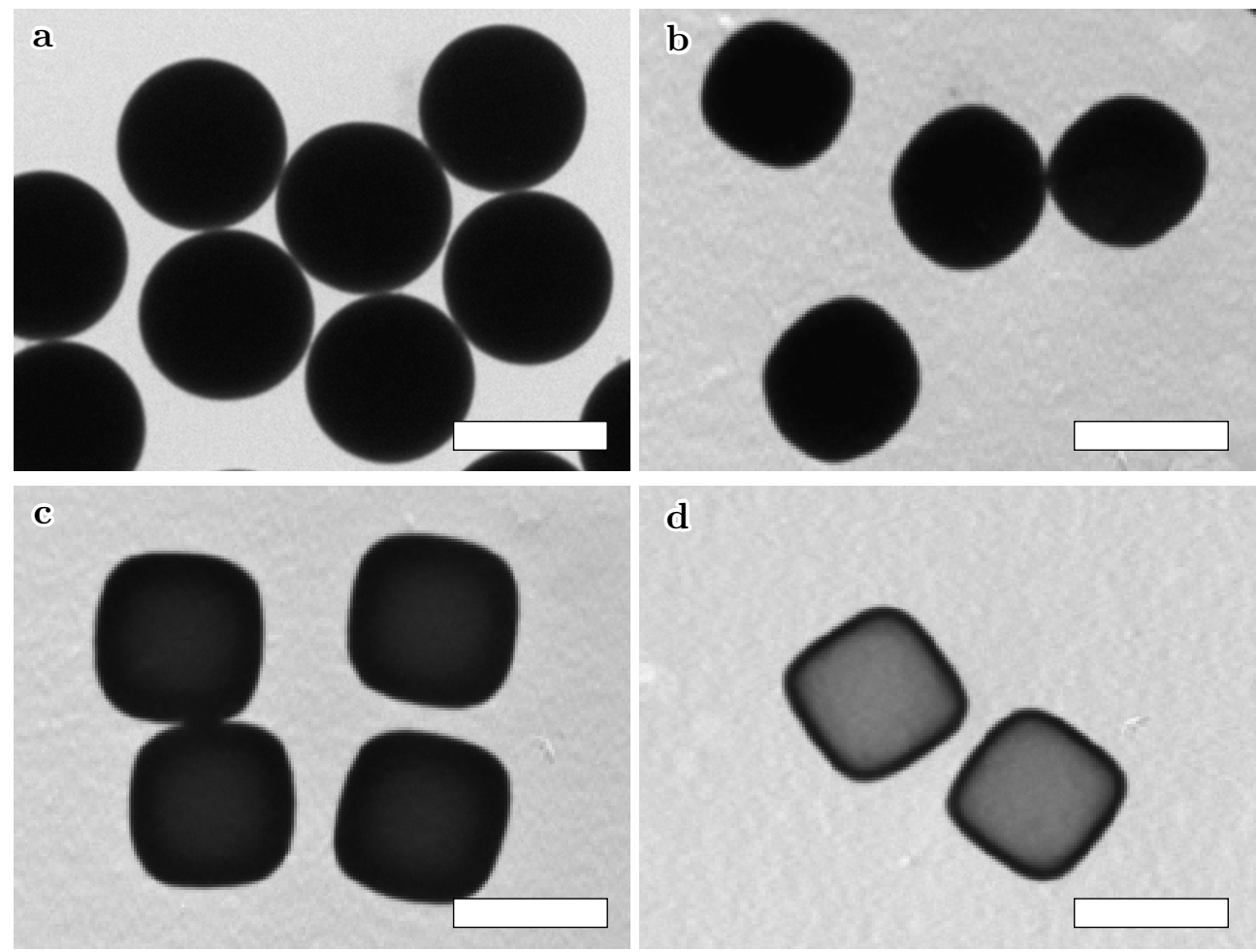

Figure 2.1: Representative transmission electron microscopy (TEM) images of colloidal silica superballs with a diameter of approximately $D=1 \mu \mathrm{m}$ and various shape parameters $m$. Their average shape parameters are $m=2$ (a), 2.87 (b), 3.32 (c) and $3.63(\mathrm{~d})$. The scale bars represent $1 \mu \mathrm{m}$.

A custom-written Python script was used in order to determine the average shape and size of the colloidal silica superballs. Here, the TEM images were first binarized using a certain threshold value. The average diameter of the spherical superballs was determined using the number of pixels of which each particle consists in the TEM images. With the known scale of the TEM images, the diameter of the particles were determined from their equivalent surface area. The shape parameter was not calculated for these spherical superballs, but instead defined as $m=2$. For the non-spherical superballs, the position of the center of mass of each of the particles was first determined as the average position of all their pixels of which each particle consists in the TEM images. The shape parameter of each particle was then calculated by first determining the distance between each pixel of the perimeter to the center of mass of the particle and its angle with respect to the center of mass of the particle. The edge pixel with the shortest distance to the particles center of mass was used to divide the angles into four quadrants. Now, for each quadrant of the particle, the distances between the center of mass and both 
the closest and furthest pixels were determined. Both the distances to the four closest and the four furthest pixels were then averaged. This allows two concentric circles with radii of $r_{1}$ and $r_{2}$ respectively to be plotted with their center in the particles' center of mass. From the definition of the contours of the superball shape, as shown in equation 1.1 on page 14, the shape parameter $m$ can be calculated from the ratio between $r_{2}$ and $r_{1}$ as follows:

$$
m=\frac{\log \left(\frac{1}{2}\right)}{\log \left(\frac{r_{2}}{\sqrt{2} r_{1}}\right)} .
$$

The edge diameter $D$ of the non-spherical superballs is defined to be twice the inner radius, or $D=2 r_{1}$. For these non-spherical superballs, only the particles that lied flat in the TEM images were considered in order to accurately determine their edge diameter $D$ and shape parameter $m$. A minimum of 50 particles was analysed per sample in order to obtain an accurate average shape and size for each of the samples. The analysis process for the non-spherical superballs is illustrated in figure 2.2 .
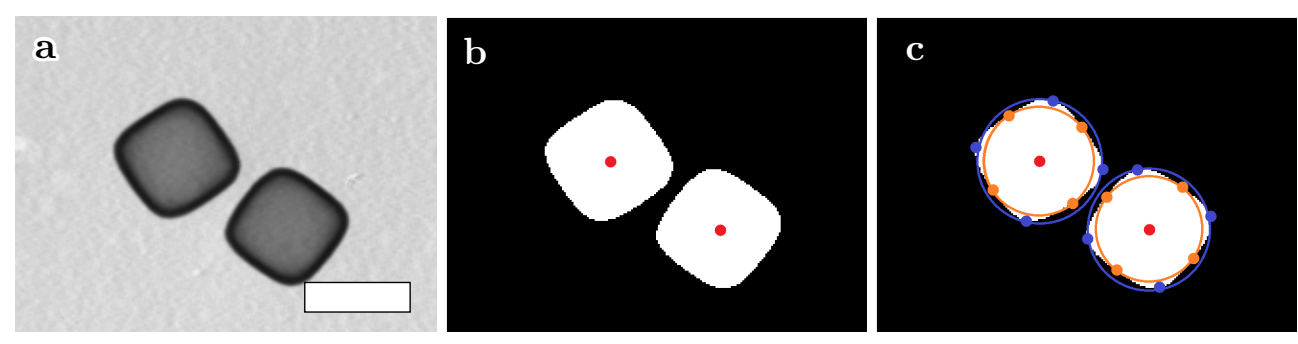

Figure 2.2: An example of the image processing of the TEM images and the determination of the average shape parameter $m$ and edge diameter $D$ of the non-spherical superballs. The TEM images are first binarized $(\mathrm{a} \rightarrow \mathrm{b})$. The center of mass of the particles (red dots) are then determined as the average position of all pixels of the particle (b). The four closest (orange dots) and four furthest (blue dots) perimeter pixels with respect to the center of mass are determined for each quadrant of the particle (c). The ratio between the average of the four closest perimeter pixels (orange circle, $r_{1}$ ) to the average of the four furthest perimeter pixels (blue circle, $r_{2}$ ) determines their shape parameter $m$. The scale bar in (a) represents $1 \mu \mathrm{m}$.

\subsection{Synthesis and characterization of monodisperse colloidal polymer spheres}

Colloidal poly(methyl methacrylate) (PMMA) spheres with a low polydispersity were synthesized for the preparation of the active colloids with anisotropic motion described in chapter 5. These colloids were prepared using the 
well-known dispersion polymerization synthesis method [7]. This easy to use synthesis method is known to be able to yield colloids with various polymer compositions reproducibly in a very wide size range [7-10]. We have chosen for PMMA due to its relatively high density of $1.18 \mathrm{~g} / \mathrm{cm}^{3}$. The high density of the polymer colloids should prevent the resulting active colloids from leaving the surface and thus assuring a two-dimensional model system. Here, $34 \mathrm{~mL}$ methanol (Acros), $10 \mathrm{~mL}$ of water (Milli-Q, $18.2 \mathrm{M} \Omega \cdot \mathrm{cm}$ ), $6 \mathrm{~mL}$ methyl methacrylate (Sigma-Aldrich) and $1.00 \mathrm{~g}$ polyvinylpyrrolidone (PVP, $M_{w}=40 \mathrm{~kg} / \mathrm{mol}$, Sigma-Aldrich) were mixed in a $100 \mathrm{~mL}$ round-bottom flask and transferred to a preheated oil bath set at a temperature of $65^{\circ} \mathrm{C}$. The round-bottom flask was capped with a rubber septum and degassed with nitrogen for a period of 10 minutes using a long needle that was pierced through the septum with the tip of the needle being below the surface level. A second needle located above the surface level was used to allow the excess of gasses to escape the flask. After degassing, a solution of $75 \mathrm{mg}$ of azobisisobutyronitrile (Sigma-Aldrich) in $10 \mathrm{~mL}$ methanol was quickly added to the flask using a syringe. The reaction was left for 20 hours, after which the flask was allowed to cool down to room temperature. The resulting colloidal PMMA spheres were washed three times with methanol using centrifugation. Small amounts of the dispersion were dried under a nitrogen flow at a time for their assembly into monolayers using the unidirectional rubbing method.

This setup allows the syntheses to be quickly and efficiently performed. Since we have found this synthesis method to be sufficiently reproducible and with the resulting polymers always being very monodisperse with polydispersities of around several percent it was not deemed necessary to remove the inhibitor present in the monomer. The average diameter of these colloids were determined in the same manner as the colloidal silica spheres described in section 2.1.3, with the exception that the TEM grids containing the PMMA colloids were sputter coated with a $10 \mathrm{~nm}$ thick layer of platinum as the electron beam of the TEM was observed to otherwise melt the bare PMMA colloids.

\subsection{Preparation of the PDMS-coated glass slides}

Polydimethylsiloxane- (PDMS) coated glass slides were prepared by first mixing the silicone elastomer base and the silicone elastomer curing agent from the Sylgard 184 silicon elastomer kit in a ratio of 10:1 by weight using vigorous stirring. The resulting mixture was then centrifuged at $3750 \mathrm{rpm}$ for 20 minutes in order to remove the air bubbles that had formed during stirring. The glass slides $(\# 0,60 \mathrm{~mm} \times 24 \mathrm{~mm} \times 0.085-0.12 \mathrm{~mm})$ were then spin coated with $1 \mathrm{~mL}$ of the PDMS mixture at $2000 \mathrm{rpm}$ for 1 minute and cured in a pre-heated oven at a temperature of $70^{\circ} \mathrm{C}$ for a period of 24 hours. 


\subsection{Device for the mechanical assembly of colloids into monolayers}

The unidirectional rubbing method relies on mechanical force to assemble colloids into monolayers $[11,12]$. While it is possible to deliver this force manually and perform the mechanical rubbing by hand, the exact speed, pressure and direction of rubbing would be difficult, if not impossible, to control. Thus, to eliminate human error, a machine should be used to achieve the most reproducible results. Unfortunately, the published work on this method did not include details of the device that was used $[11,12]$. In this section we present the custom-made prototype device that we have designed for the mechanical assembly of colloids into monolayers using the unidirectional rubbing method, and share how this device can be build and operated. This machine allows the direction of rubbing and both the pressure and speed at which the colloids are rubbed onto the surface to be precisely controlled. The design of this device relies exclusively on readily available off-the-shelves parts and a 3D printed body and should be able to be replicated without extended technical knowledge or specialized equipment. The 3D printing can also be outsourced easily, as there are many online 3D printing services. The total cost of the materials is roughly $€ 80$. The electronics of the device are based on an Arduino Uno microcontroller which are easy to program. The bill of materials, programming code and 3D printing files are available upon request. A photograph of the assembled device is depicted in figure 2.3.

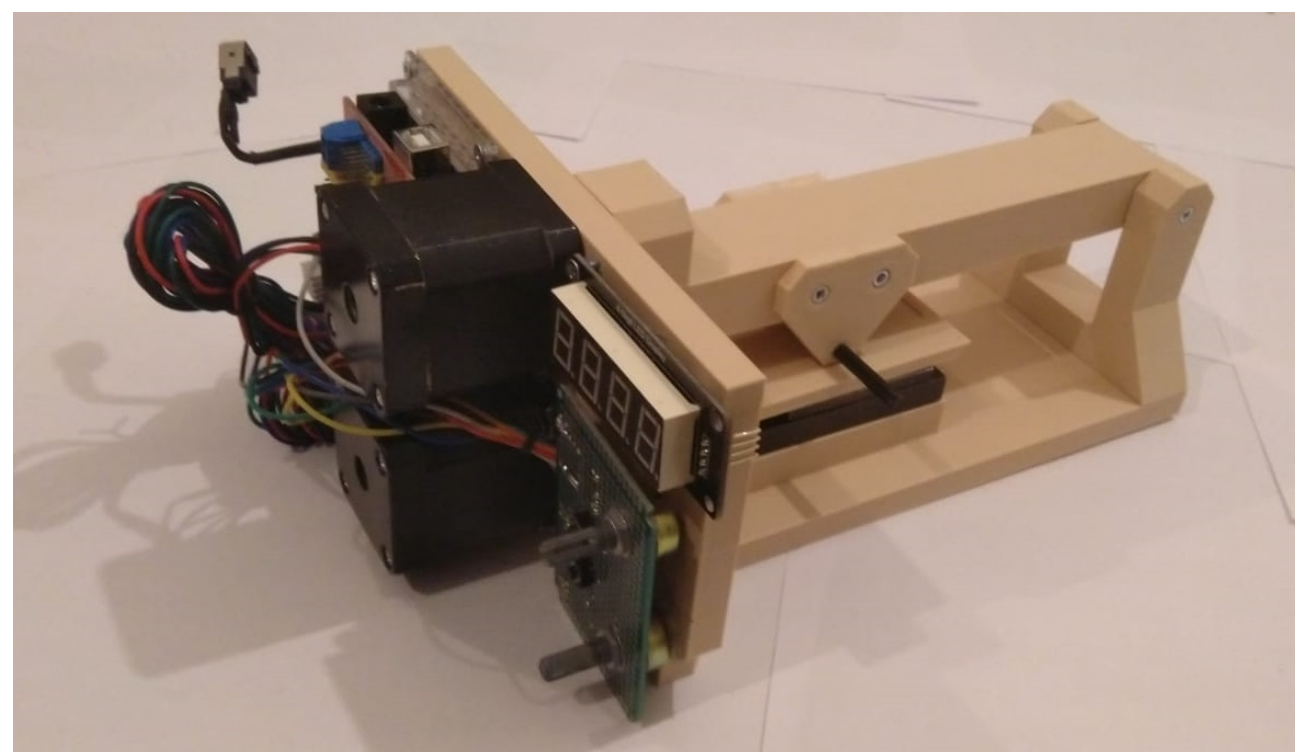

Figure 2.3: The custom-made prototype device for the mechanical assembly of colloids in monolayers using the unidirectional rubbing method. 
The 3D printed parts are screwed together using M3 countersunk screws and form the rigid body of the device to which the rest of the components are attached to. An Arduino Uno equipped with the Arduino CNC Shield V3 and two A4988 stepper motor drivers were used to power the two generic NEMA 17 stepper motors. The carriage features a recess that is $76 \mathrm{~mm} \times 24 \mathrm{~mm} \times$ $0.5 \mathrm{~mm}$ in size which fits common microscopy glass slides of that length and width. For the preparation of colloidal monolayers on thinner glass slides, such as \#0 and \#1 glass slides, a thick slide that is coated with a layer of PDMS can be placed in the recess to provide grip for the thinner slide that is laying on top of it. The friction between the two glass slides is strong enough for the thin slide to remain in place during its preparation. An M5 nut is inserted into the carriage so that a threaded rod of the same size can be used to move the carriage. The threaded rod is connected to the stepper motor using a motor coupling. Two potentiometers allow the rubbing speed and the amount of rubbing cycles to be set. A 4 digit segment display (TM1637) is used to show the values of these parameters. The rubbing process can then be started by pressing a button. Finally, an end stop provides a reference point to determine the starting point for the back and forth rubbing motion. The 3D printed arm of the device is equipped with a cylinder of PDMS $(D=1 \mathrm{~cm})$ which is rubbed over the sample. These PDMS cylinders can be made by filling a small centrifuge tube with PDMS using the same preparation as the PDMS-coated glass slides described in section 2.3. Here, a LEGO axle is inserted before curing which allows the cylinder to be conveniently mounted to the arm of the device. The second stepper motor is used to lift the arm. Weights can be placed on top of the arm to increase the pressure on the arm. The pressure exerted by the PDMS-cylinder on the sample can measured and calibrated using a scale by varying the mass and the position of the weight. While the color of the 3D printed parts is arbitrary, printing it in a color which provides some contrast to the color of the particles of choice may be useful as it aids the ability the properly clean the device after use.

The device is programmed as such that the PDMS cylinder is lifted over the remaining colloidal powder after each shove, before it continues rubbing in the opposite direction. This allows the remaining colloidal powder to remain being shoved over the colloidal monolayer, which should fill the voids in the monolayer and increase its packing as much as possible. The trajectory of the PDMS-cylinder is schematically depicted in figure 2.4a. After the machine is finished, the excess colloidal powder can be blown off using a strong air or nitrogen flow. This can be done without disturbing the colloidal monolayer as the particles are more strongly attached to the PMDS-coated substrate than to other particles [11]. This method thus ensures the formation of a purely two-dimensional monolayer with no extra particles or secondary layers. The device was tested by assembling spherical polystyrene colloids with an average diameter of $D=1.0 \mu \mathrm{m} \pm 2.0 \%$ into colloidal monolayers. A representative 
scanning electron microscopy (SEM) image of this colloidal monolayer is depicted in figure 2.4b. It can be seen here that the resulting colloidal monolayer has a very well-defined lattice structure with all crystal grains aligned in the direction of rubbing, which was the horizontal direction in the image. We have found that 10 rubbing cycles was enough for these colloidal monolayers to be prepared reproducibly. Finally, we thus concluded that the device functioned as desired.

a

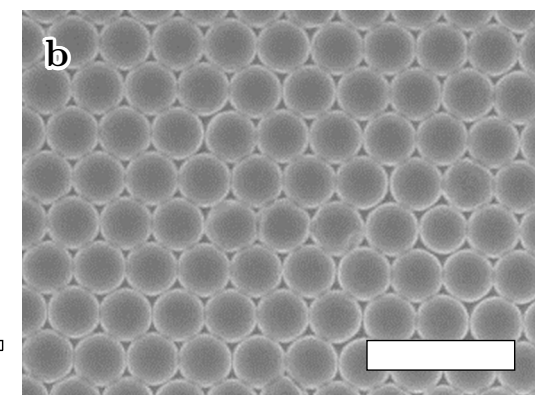

Figure 2.4: A schematic representation of the trajectory of the PDMS cylinder which rubs the colloidal powder over the PDMS-coated subtrate (a). This schematic representation is drawn in a 1:1 scale. A representative SEM image of a monolayer of colloidal polystyrene spheres with an average diameter of $D=1.0 \mu \mathrm{m}$ and a polydispersity of $D_{\sigma}=2.0 \%$ assembled using the custom-made prototype device (b). The mechanical rubbing was applied in the horizontal direction of the image. The scale bar in (b) represents $3 \mu \mathrm{m}$.

\subsection{Cells to study colloidal dispersions under the influence of an alternating electric field}

In order to study the colloidal dispersions containing the active colloids under the influence of an alternating electric field, a potential difference needs to be applied between two electrodes. The magnitude of the resulting alternating electric field, $E$, is determined by the applied potential difference, $\delta V$, and the spacing between the electrodes, $d$, as follows:

$$
E=-\frac{\delta V}{d} \text {. }
$$

In this thesis we present the magnitude of the alternating electric field as the peak-to-peak voltage present between the electrodes, or $E_{p t p}$. We have used custom-made cells to study the active colloids under the influence of an alternating electric field in situ using optical microscopy. These cells consisted of two indium tin oxide (ITO) -coated glass slides that were spaced $100 \mu \mathrm{m}$ 
apart with the ITO-coating on the inside of the cells. We have deliberately used a small spacing between the electrodes in order to minimize the distortion in the electric field that occurs near the edge of the electrodes. In addition, the relatively small spacing between the ITO-surfaces results in only a relatively small potential difference required between the electrodes in order to obtain an alternating electric field of equal magnitude. We have found that this minimizes the presence of unwanted side reactions and flows which would disturb the measurements. The wires are attached to the ITO-coated glass slides using conductive silver loaded epoxy glue. The front and rear side of the cell are open, which allows the cells to be filled using capillary action. These cells are reusable and can be cleaned by placing them in ethanol and drying them under a nitrogen flow. A schematic representation and a photograph of the alternating electric field cells are depicted in figure $2.5 \mathrm{a}$ and $\mathrm{b}$ respectively.

a

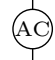

b

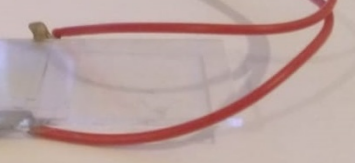

Figure 2.5: A schematic representation (a) and a photograph (b) of the custommade alternating electric field cells that were used to study the active colloids under the influence of an alternating electric field. An alternating electric field is applied between the two conductive ITO-coated glass slides that are spaced approximately $100 \mathrm{\mu m}$ apart with the conductive ITO surfaces on the inside of the cell and in direct contact with the colloidal dispersion.

\subsection{Acknowledgements}

Dominique Thies-Weesie is thanked for her help with the preparation of the colloidal silica spheres. Faranaaz Rogier and Bonny Kuipers are thanked for the fruitful discussions on making cells with an applied alternating electric field.

\section{References}

[1] W. Stöber, A. Fink, and E. Bohn, "Controlled growth of monodisperse silica spheres in the micron size range," Journal of Colloid and Interface Science, vol. 26, pp. 62-69, 1968. 
[2] X. Lei, B. Yu, H. L. Cong, C. Tian, Y. Z. Wang, Q. B. Wang, and C. K. Liu, "Synthesis of monodisperse silica microspheres by a modified stöber method," Integrated Ferroelectrics, vol. 154, no. 1, pp. 142-146, 2014.

[3] S. Zhang, G. L. Li, H. L. Cong, B. Yu, and X. Y. Gai, "Size control of monodisperse silica particles by modified Stöber method," Integrated Ferroelectrics, vol. 178 , no. 1, pp. 52-57, 2017.

[4] T. Sugimoto and T. Sakata, "Preparation of monodisperse pseudocubic $\alpha$ Fe2O3 particles from condensed ferric hydroxide gel," Journal of Colloid And Interface Science, vol. 152, no. 2, pp. 587-590, 1992.

[5] L. Rossi, S. Sacanna, W. T. Irvine, P. M. Chaikin, D. J. Pine, and A. P. Philipse, "Cubic crystals from cubic colloids," Soft Matter, vol. 7, no. 9, pp. 4139-4142, 2011.

[6] T. Sugimoto, K. Sakata, and A. Muramatsu, Formation mechanism of monodisperse pseudocubic $\alpha$-Fe2O3 particles from condensed ferric hydroxide gel, 1993.

[7] K. Cao, J. Yu, B. G. Li, B. F. Li, and Z. R. Pan, "Micron-size uniform poly(methyl methacrylate) particles by dispersion polymerization in polar media 1. Particle size and particle size distribution," Chemical Engineering Journal, vol. 78, no. 2-3, pp. 211-215, 2000.

[8] Y. Yamada, T. Sakamoto, S. Gu, and M. Konno, "Soap-free synthesis for producing highly monodisperse, micrometer-sized polystyrene particles up to 6 $\mu \mathrm{m}, "$ Journal of Colloid and Interface Science, vol. 281, no. 1, pp. 249-252, 2005.

[9] Q. Liu, L. Wang, A. Xiao, H. Yu, Q. Tan, J. Ding, and C. Yu, "Controllable preparation of monodisperse polystyrene microspheres with different sizes by dispersion polymerization," Macromolecular Symposia, vol. 261, no. 1, pp. 113$120,2008$.

[10] C. W. Chen and C. Y. Chen, "Preparation of monodisperse polystyrene microspheres: Effect of reaction parameters on particle formation, and optical performances of its diffusive agent application," Colloid and Polymer Science, vol. 287, no. 12, pp. 1377-1389, 2009.

[11] C. Park, T. Lee, Y. Xia, T. J. Shin, J. Myoung, and U. Jeong, "Quick, large-area assembly of a single-crystal monolayer of spherical particles by unidirectional rubbing," Advanced Materials, vol. 26, no. 27, pp. 4633-4638, 2014.

[12] C. Park, K. Koh, and U. Jeong, "Structural Color Painting by Rubbing Particle Powder," Scientific Reports, vol. 5, pp. 1-5, 2015. 


\section{Chapter 3}

\section{Form Factor Effects in Colloidal Superball Monolayers}

\section{Abstract}

Small-angle X-ray scattering (SAXS) is a powerful tool for the structural characterization on the scale of nanometers to about a micrometer. The analysis of the resulting diffraction patterns can, however, be complicated due to Bragg peaks missing as a result of minima present in the particles' form factor. In this chapter, we present a strategy for the characterization of monolayers of colloidal silica superballs prepared using the unidirectional rubbing method using SAXS. Using this assembly method the colloidal superballs were mechanically rubbed onto a polydimethylsiloxane- (PDMS) coated surface. Using three shapes of colloidal superballs, ranging from spherical to almost cubic, we show that these missing Bragg peaks can be visualized by imaging the colloidal superball monolayers at various orientations, which allows a complete analysis. Finally, we present a model for the structure factor of these colloidal superball monolayers and discuss the transition in the resulting lattices observed upon varying the shape of the superballs from spherical to almost cubic. 


\subsection{Introduction}

Colloidal monolayers are used in a wide variety of applications such as antireflective coatings, photonic materials, photovoltaics and biosensensors $[1,2]$. Alternatively, the assembly of colloids into monolayers is often used as a step in colloidal syntheses in order to locally modify the particles such as, for example, to produce Janus colloids [3-5]. Various methods are known for the preparation of colloidal monolayers such as drying methods, spin coating, the assembly on liquid interfaces and the horizontal and vertical deposition method [2]. Each of these preparation methods have their own strengths and weaknesses. It was only recently shown, however, that colloidal monolayers could also be prepared by mechanically rubbing dried colloids between two sticky surfaces such as polydimethylsiloxane (PDMS) [6, 7]. This quick, simple and inexpensive method produces high-quality colloidal monolayers where the colloidal crystal grains align in the direction in which the mechanical rubbing was applied.

Until now, the unidirectional rubbing method has only been used for the assembly of spherical particles into monolayers. The shape of the individual particles can, however, greatly influence the structures that are being formed [8]. This may result in the colloidal monolayers having different properties that might be of interest in one of their many applications. Interesting phase behaviour has been observed, for example, upon allowing superball-shaped colloids to sediment $[9,10]$ and upon assembling these particles in monolayers using the horizontal and vertical deposition method [11, 12]. The variable shape of the superballs allows smooth phase transitions to occur.

In this thesis we have prepared monolayers of colloidal silica superballs with various shapes and sizes using the unidirectional rubbing method in order to determine the influence of their shape and size on the resulting lattice structures. We have used small-angle X-ray scattering (SAXS) to analyse these lattice structures as this method allows for the characterization of long-range order on the colloidal scale. In addition, compared to for example microscopy techniques, SAXS captures and averages data from over a relatively large area of the sample, therefore obtaining a more representative picture of the sample [13]. In SAXS the obtained data does not only depend on the assembly of the particles but also on the shape of the individual particles. The latter is described by the form factor. For spheres the form factor is isotropic as spheres scatter X-rays equally in all directions and the form factor can be calculated analytically. For all other shapes, however, the form factor is not isotropic which complicates the analysis of the resulting scattering patterns. Furthermore, the presence of minima in the form factor may cause Bragg peaks to become less visible or completely invisible in the diffraction patterns. 
In this chapter we present a strategy for the measurement and analysis of the SAXS diffraction patterns of monolayers of colloidal silica superballs prepared using the unidirectional rubbing method. We show that by imaging the colloidal monolayers under a range of rotations a more detailed analysis can be preformed. Here, by rotating the sample, the $q$-values of the Bragg peaks in the diffraction pattern can be shifted, therefore visualizing any Bragg peaks coinciding with minima present in the form factor. The effect of the sample being rotated is first illustrated using spheres of which the form factor is isotropic and can be calculated analytically. We then show that for two more cubic superballs, referred to here as semi-cubic and cube-like superballs and of which the form factor cannot be calculated analytically, we are able to visualise and assign all Bragg peaks for a correct and complete analysis. Finally, we present a model for the transition observed in the lattices of colloidal superball monolayers prepared using the unidirectional rubbing method upon varying the shape of the colloidal superballs from spherical to almost cubic.

\subsection{Theory}

In SAXS the scattered intensity is the product of the form factor and the structure factor:

$$
I(\vec{q})=P(\vec{q}) S(\vec{q}),
$$

where $\vec{q}$ is the scattering vector. The form factor $P(\vec{q})$ is determined by the shape and size of the individual particles. For uniform spherical particles the form factor is radially symmetric and equal to:

$$
P(\vec{q})=P(q)=\frac{3[\sin (q R)-(q R) \cos (q R)]}{(q R)^{3}},
$$

where $q$ is the length of the scattering vector and $R$ is the radius of the spherical particle. Due to the symmetry of the spherical shape the form factor does not depend on its orientation. For non-spherical particles, however, the form factor does depend on the orientation of the particle. While there are no analytical solutions for the form factor for most anisotropic shapes, they can always be calculated numerically.

The structure factor $S(\vec{q})$, on the other hand, is determined by the structural assembly of the particles. Consider an ideal two-dimensional lattice with lattice points at distance vectors of:

$$
\vec{r}=m \vec{a}_{1}+n \vec{a}_{2},
$$

where $m$ and $n$ are integers and $\vec{a}_{1}$ and $\vec{a}_{2}$ are the primitive lattice vectors. 
The structure factor of any structural assembly is equal to:

$$
S(\vec{q})=\frac{1}{N}\left|\sum_{n=1}^{N} e^{i \vec{q} \cdot \vec{r}_{n}}\right|^{2},
$$

where $N$ is the total number of particles and $\vec{r}_{n}$ is the distance vector of the $n$th particle. For an ideal two-dimensional lattice the structure factor is non-zero at:

$$
\vec{q}=h \vec{b}_{1}+k \vec{b}_{2}
$$

for any value of $q_{z}$. Here $h$ and $k$ are the Miller indices, $\vec{b}_{1}$ and $\vec{b}_{2}$ are the primitive reciprocal basis vectors and $q_{z}$ is the component of the scattering vector that is parallel to the incoming X-ray beam. The angle between these primitive reciprocal basis vectors is defined as $\beta$. The primitive reciprocal basis vectors are related to the primitive lattice vectors $\vec{a}_{1}$ and $\vec{a}_{2}$ as follows:

$$
\vec{b}_{1}=2 \pi \frac{\vec{a}_{2} \times \vec{n}}{\left|\vec{a}_{1} \times \vec{a}_{2}\right|}, \vec{b}_{2}=2 \pi \frac{\vec{n} \times \vec{a}_{1}}{\left|\vec{a}_{1} \times \vec{a}_{2}\right|},
$$

where $\vec{n}$ is a vector normal to the two-dimensional lattice plane. The angle between the primitive lattice vectors is $\alpha=180^{\circ}-\beta$. Alternatively, by rewriting equations 3.6, the lengths of the primitive lattice vectors can be calculated as:

$$
a_{1}=\frac{2 \pi}{b_{1} \sin \beta}, a_{2}=\frac{2 \pi}{b_{2} \sin \beta},
$$

where $b_{1}$ and $b_{2}$ are the lengths of the primitive reciprocal basis vectors $\vec{b}_{1}$ and $\vec{b}_{2}$ respectively.

\subsection{Experimental methods}

\subsubsection{Preparation of the colloidal superball monolayers}

The colloidal silica spheres were synthesized using the Stöber process as described in subsection 2.1.1. The hollow silica superballs were prepared by coating hematite cubes with a layer of silica using a modified Stöber process and subsequently dissolving the hematite core as described in subsection 2.1.2. The average shape parameter $m$ and edge diameter $D$ of the resulting superball colloids were determined using a custom-written Python script from transmission electron microscopy (TEM) images as shown in subsection 2.1.3. The spherical, semi-cubic and cube-like superballs described in this chapter have an average shape parameter of $m=2,2.87$ and 3.63 and edge diameter of $D=1.10,0.96$ and $1.01 \mu \mathrm{m}$ respectively. Representative TEM images of these superball-shaped colloids are depicted in figure $2.1 \mathrm{a}, \mathrm{b}$ and $\mathrm{d}$ respectively. The 
colloidal silica superballs were assembled into monolayers by mechanically rubbing them onto polydimethylsiloxane- (PDMS) coated glass slides $(\# 0,60 \mathrm{~mm}$ x $24 \mathrm{~mm} \times 0.085-0.12 \mathrm{~mm}$ ) using the custom-made device presented in section 2.4. For all three samples the device was set to run 10 rubbing cycles at a rubbing pressure of $200 \mathrm{~g}$ and a rubbing speed of $3 \mathrm{~mm} / \mathrm{s}$.

\subsubsection{Analysis of the colloidal superball monolayers using SAXS}

The SAXS measurements were performed at the BM26B DUBBLE beamline of the ESRF in Grenoble, France. The X-ray beam with a photon energy of $12 \mathrm{kV}$ was focused on a CCD with a resolution of $4008 \times 2671$ pixels and pixel size of $22 \times 22 \mu \mathrm{m}$ (Photonic Science) placed at a distance of $7.5 \mathrm{~m}$ from the sample. An exposure time of $20 \mathrm{~s}$ was used for all measurements. A wedgeshaped beam-stop was used in order to prevent damage to the detector that can result from an exposure to the direct X-ray beam. The SAXS setup is schematically depicted in figure 3.1 .

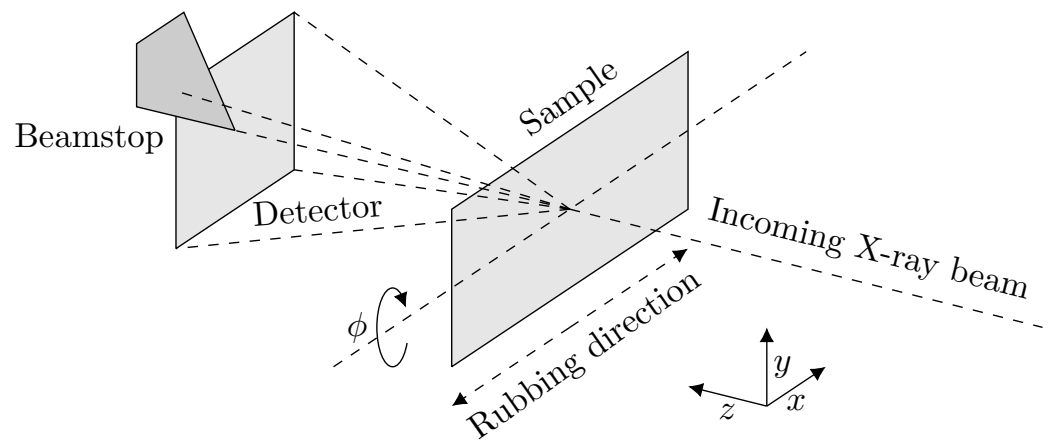

Figure 3.1: A schematic representation of the small-angle X-ray scattering (SAXS) setup that was used. Here the sample, a monolayer of colloidal superballs, can be rotated around the $x$-axis. The angle of rotation around this axis is denoted by $\phi$. The incoming X-ray beam travels along the $z$-axis. The scattering pattern is imaged in $x, y$-space. A beamstop was used to prevent damage to the detector. Note that this schematic representation is not drawn to scale.

This setup allows the samples to be rotated from $\phi=-60$ to $60^{\circ}$. Blank PDMS-coated glass slide were also imaged at the various rotations $\phi$ for background corrections. Every measured diffraction pattern image was subtracted with the background imaged at an equal $\phi$ to remove the scattering contributions from the PDMS-coated glass slide. Custom-written Python scripts were used to determine the positions of the peaks in the diffraction pattern images and their cross sections and to numerically calculate the form factor of the superball colloids. Dried collagen fiber extracted from a rat tail was used for the calibration of the $q$-values. The resolution of the diffraction patterns was determined to be $0.180 \mathrm{um}^{-1} /$ pixel from the position of the well-defined first order ring which corresponds to a real-space distance of $67 \mathrm{~nm}$. 


\subsection{Results and discussion}

We begin by discussing the SAXS diffraction patterns of a monolayer of solid spherical silica superballs with an average diameter of $D=1.10 \mu \mathrm{m} \pm 3.2 \%$ prepared using the unidirectional rubbing method to illustrate the effect of the sample rotation. Diffraction patterns imaged at rotations around the $x$-axis of $\phi=0,20$ and $40^{\circ}$ are depicted in figure $3.2 \mathrm{a}$, b and c respectively. In all of these diffraction patterns sharp Bragg peaks can be seen which indicate that the spheres had formed well-defined periodic structures. The presence of separate peaks, instead of a series of co-axial rings, indicate that the crystal orientation within the imaged area of the colloidal monolayer was fixed. This is a known result of the unidirectional rubbing method where the crystal grains align in the direction of rubbing $[6,7]$.

In the diffraction pattern imaged at a rotation of $\phi=0^{\circ}$, depicted in figure $3.2 \mathrm{a}$, it can be seen that the Bragg peaks have six-fold symmetry which indicates that the crystal structure of the colloidal monolayer is a two-dimensional hexagonal lattice. Some of the Bragg peaks we use for their characterization are labelled in the diffraction patterns depicted in figure $3.2 \mathrm{a}$, b and c. Upon rotating the sample around the $x$-axis the Bragg peaks shift in the vertical $y$ direction which makes the diffraction patterns appear increasingly elongated. In addition, it can be seen that the intensity of the peaks in the diffraction patterns change relative to each other. The 10 peak is strongly visible at $\phi=0$ and $20^{\circ}$, but disappears at $40^{\circ}$. Similarly, the 20 peak is visible at $\phi=0^{\circ}$, disappears at $20^{\circ}$ and becomes visible again at $40^{\circ}$ while the 30 peak is not visible at $\phi=0^{\circ}$, only slightly visible at $20^{\circ}$ and disappears again at $40^{\circ}$. These trends can be more easily visualized using the cross sections of the scattering patterns in the vertical direction at $q_{x}=0 \mu \mathrm{m}^{-1}$. The vertical cross sections of the diffraction patterns imaged at rotations around the $x$-axis of $\phi=0,10$, 20,30 and $40^{\circ}$ at $q_{x}=0 \mu^{-1}$ are depicted in figure $3.2 \mathrm{~d}$. Here, the scattering vector $q_{y}$ is scaled by a factor of $\cos (\phi)$ in order to align all Bragg peaks in the various cross sections at equal values of $q_{y} \cos (\phi)$. The position of the 10,20 and 30 peaks are marked in the plot. Thus, by determining the cross sections of the diffraction patterns a more precise analysis can be performed to locate the positions of the various Bragg peaks. In this case it can be seen that the 10,20 and 30 peaks occur at $q_{y} \cos (\phi)=5.95,11.90$ and $17.85 \mathrm{\mu m}^{-1}$.

The strong changes in the relative intensity of the Bragg peaks observed upon rotating the sample around the $x$-axis result from the presence of minima in the form factor. Upon rotating the sample around the $x$-axis, the distances of periodicity in the sample in the $y$-direction decrease and thus correspond to higher $q_{y}$-values in reciprocal space. This process is depicted in figure $3.2 \mathrm{e}$ 
which shows the form factor of these spheres, calculated using equation 3.2, along with the positions of the 10,20 and 30 peaks at rotations around the $x$ axis of $\phi=0,10,20,30$ and $40^{\circ}$. It can be seen that upon rotating the sample around the $x$-axis the positions of various peaks shift to larger $q_{y}$-values which results in changes in their relative intensities. This example shows that a large enough range in the scattering vector $q_{y}$ can be probed to shift the positions of the 10, 20 and 30 peaks away from the minima present in the form factor to visualize and identity these Bragg peaks. Similar analyses can be performed to determine the positions of the other Bragg peaks present in the diffraction pattern. The 01 peak, for example, is visible in each of the diffraction patterns depicted in figure $3.2 \mathrm{a}, \mathrm{b}$ and $\mathrm{c}$, whereas both the 11 and 21 peaks are only visible up to a rotation of $\phi=20^{\circ}$.
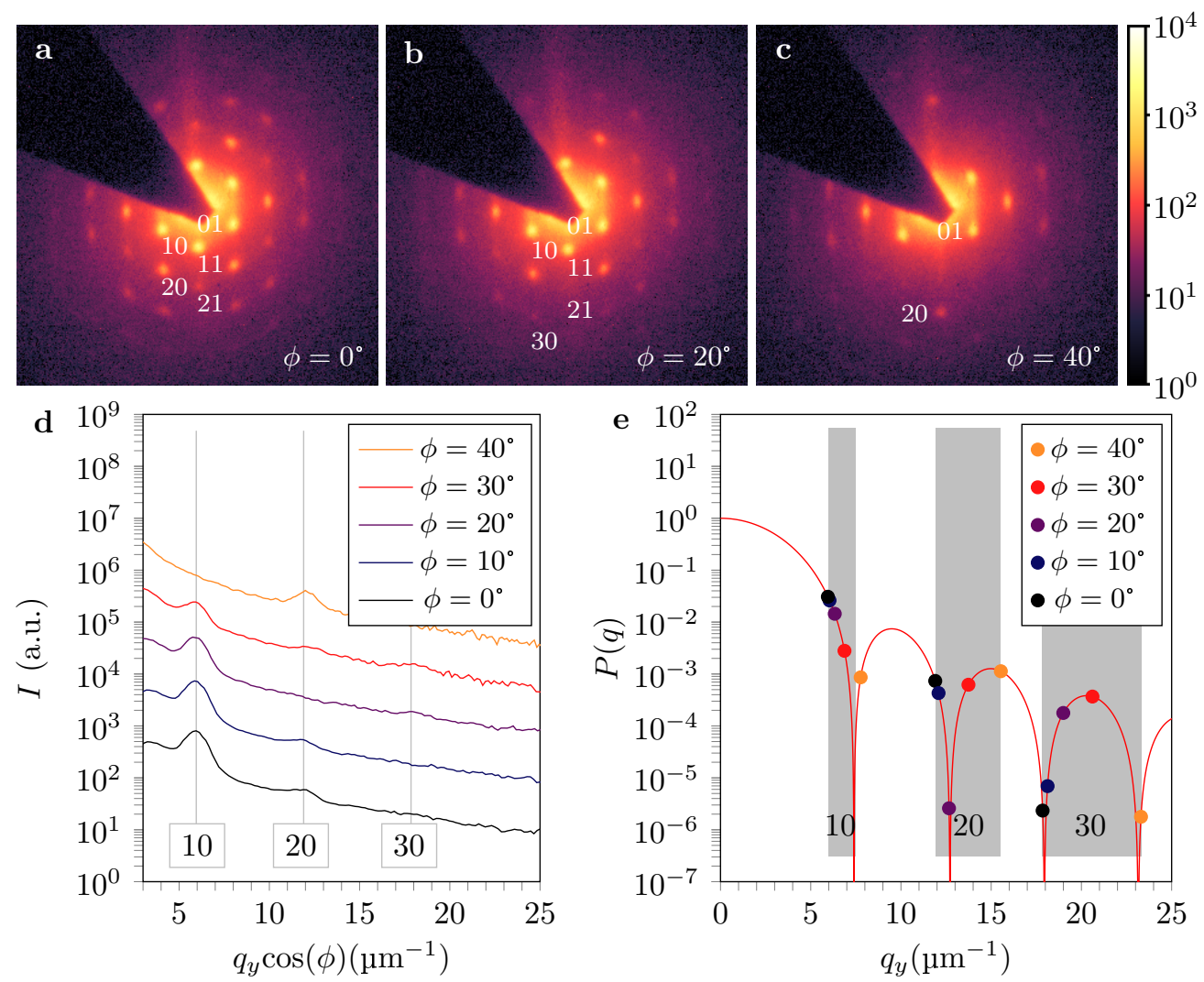

Figure 3.2: SAXS diffraction patterns of a monolayer of colloidal silica spheres with an average diameter of $D=1.10 \mu \mathrm{m} \pm 3.2 \%$ imaged at rotations around the $x$-axis of $\phi=0$ (a), 20 (b) and $40^{\circ}$ (c). The Bragg peaks are labelled on their left. Cross sections of the diffraction patterns taken at $q_{x}=0 \mu \mathrm{m}^{-1}$ at rotations of $\phi=0,10$, 20,30 and $40^{\circ}$ (d). The positions of the identified peaks are visualized in the cross sections at the corresponding $q_{y} \cos (\phi)$ positions. The calculated form factor of these spheres along with the positions of the 10,20 and 30 at the various rotations $\phi(e)$. 
We continue by discussing the diffraction patterns of a monolayer of the hollow semi-cubic superballs which have an average shape parameter of $m=$ $2.87 \pm 13 \%$ and edge diameter of $D=0.96 \mu \mathrm{m} \pm 4.5 \%$. Diffraction patterns imaged at rotations of $\phi=0,20$ and $40^{\circ}$ are depicted in figure $3.3 \mathrm{a}, \mathrm{b}$ and c respectively. Again, distinct Bragg peaks can be observed which can be identified as shown in figure $3.3 \mathrm{a}, \mathrm{b}$ and $\mathrm{c}$. In figure $3.3 \mathrm{a}$ it can be seen that at $\phi=0^{\circ}$ the 10 peak is only barely visible with both the 20 and 30 peaks more clearly visible. At $\phi=20^{\circ}$, depicted in 3.3b, no peaks along the vertical axis at $q_{x}=0 \mathrm{um}^{-1}$ are significantly visible. At $\phi=40^{\circ}$, depicted in figure 3.3c, the 10 and 20 peaks are visible while the 30 peak is not visible at all.
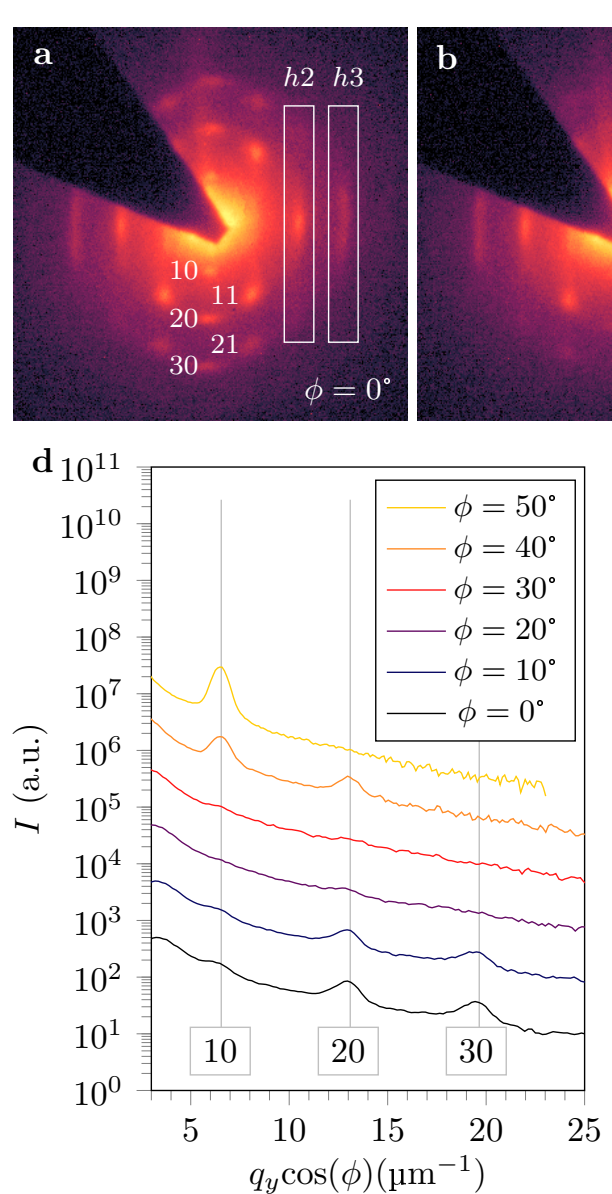
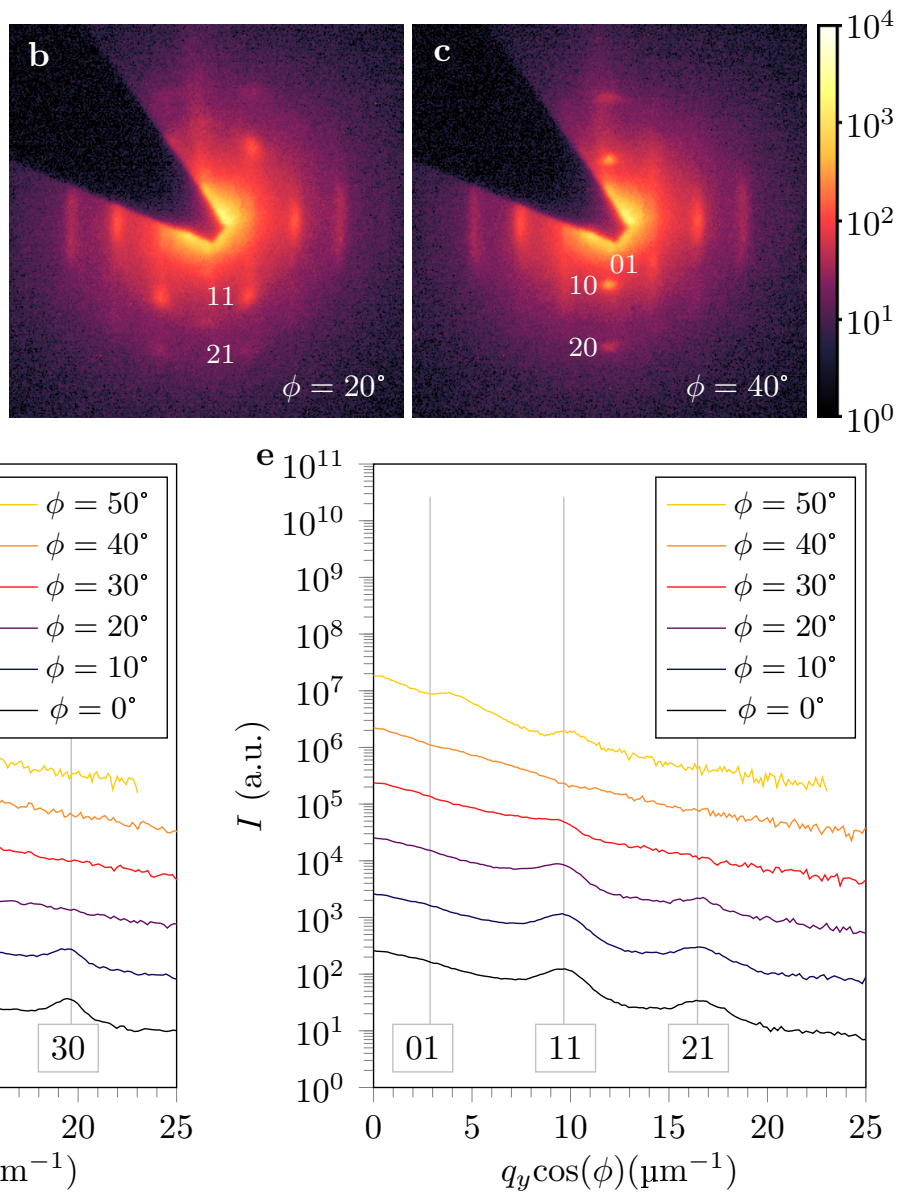

Figure 3.3: SAXS diffraction patterns of a monolayer of hollow semi-cubic colloidal silica superballs with an average shape parameter of $m=2.87 \pm 13 \%$ and edge diameter of $D=0.96 \mu \mathrm{m} \pm 4.5 \%$ imaged at rotations around the $x$-axis of $\phi=0$ (a), 20 (b) and $40^{\circ}$ (c). The Bragg peaks are labelled on their left. Cross sections of the diffraction patterns at $q_{x}=0 \mu^{-1}$ (d) and $q_{x}=6.12 \mu \mathrm{m}^{-1}$ (e). The positions of the identified peaks are visualized in the cross sections at the corresponding $q_{x} \cos (\phi)$ positions. 
The vertical cross sections of the diffraction patterns imaged at the various rotations of $\phi$ at $q_{x}=0 \mu^{-1}$ are depicted in figure 3.3d. Here, it can be seen that the 10 peak is only visible at high $\phi$-values, while the 20 and 30 peaks are only visible at low $\phi$-values and that no peaks are observed at rotations in the $x$-axis of $\phi=20$ and $30^{\circ}$. It is clear that the angles at which the various Bragg peaks are visible in the diffraction patterns are different than those of the solid silica spheres as a result of their different form factors. To further understand and characterize these non-trivial SAXS diffraction patterns it is useful to also consider vertical cross sections at different positions of $q_{x}$. The vertical cross sections of the diffraction patterns imaged at the various rotations of $\phi$ at $q_{x}=6.12 \mu^{-1}$ are depicted in figure 3.3e. These cross sections contain the $h 1$ peaks as illustrated in figure 3.3a. Here, the 01 peak becomes visible only at a rotation around the $x$-axis of $\phi=50^{\circ}$. The 11 and 21 peaks are both visible at up to rotations in the $x$-axis of $\phi=20^{\circ}$ and disappear at higher rotation angles. The 11 peak becomes slightly visible in the cross sections again at $\phi=50^{\circ}$. Finally, it can be seen that the $h 1, h 2$ and $h 3$ peaks become increasingly broadened in the vertical $y$-direction to the point where the $h 3$ peaks appear to form a single continuous band. The width of these peaks, however, does not appear to broaden. We will discuss the cause and implications of these observations later in this chapter.

An even more striking influence of the particles' form factor can be observed in the diffraction patterns of a monolayer of the hollow cube-like superballs which have an average shape parameter of $m=3.63 \pm 12 \%$ and edge diameter of $D=1.01 \mu \mathrm{m} \pm 6.6 \%$. Their diffraction patterns imaged at rotations around the $x$-axis of $\phi=0,20$ and $40^{\circ}$ are depicted in figure $3.4 \mathrm{a}$, b and $\mathrm{c}$ respectively. Here, not even a single Bragg peak is significantly visible in the diffraction pattern imaged at $\phi=0^{\circ}$. Upon rotating the sample around the $x$-axis, however, some of the diffraction peaks can be made visible as depicted and identified in figure $3.4 \mathrm{~b}$ and $\mathrm{c}$. The vertical cross sections of the diffraction patterns at $q_{x}=0 \mu \mathrm{m}^{-1}$ are depicted in figure $3.4 \mathrm{~d}$. Here, the 10 peak is clearly visible at rotations around the $x$-axis of $\phi=30^{\circ}$ and larger, while the 30 peak is only visible at $\phi=30^{\circ}$. The 20 peak becomes visible at $\phi=10^{\circ}$, disappears at $\phi=40^{\circ}$ and becomes visible again at $\phi=50^{\circ}$. Furthermore, broadened and almost continuous bands can be seen again which can be ascribed to the $h 1$ and $h 2$ peaks as shown in figure 3.3a. In figure $3.4 \mathrm{e}$ a second cross section of the diffraction patterns taken at $q_{x}=5.76 \mu^{-1}$ is depicted, corresponding to the intensity ascribed to the $h 1$ peaks. While two very broad maxima can vaguely be seen at around $q_{y} \cos (\phi)=13 \mu \mathrm{m}^{-1}$ and $20 \mu \mathrm{m}^{-1}$ at the various rotations around the $x$-axis, these apparent maxima may also be a result of the form factor which might split up a continuous band. Therefore, no individual can be assigned here. Similarly to the diffraction patterns of the monolayers of the semi-cubic superballs, the Bragg peaks only broaden in the vertical direction. 

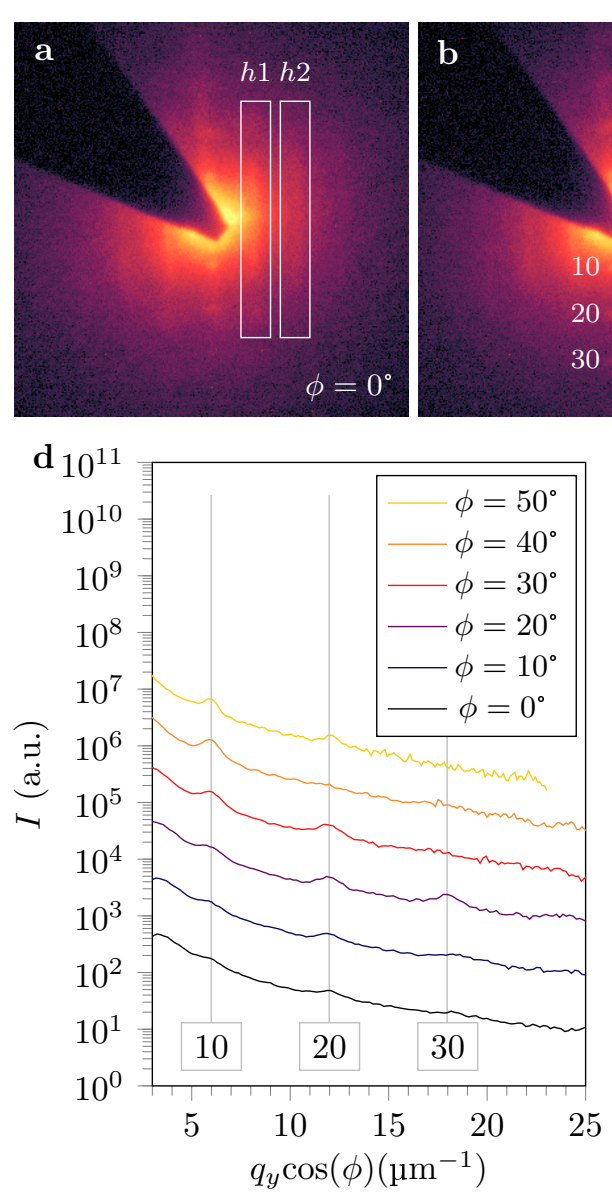
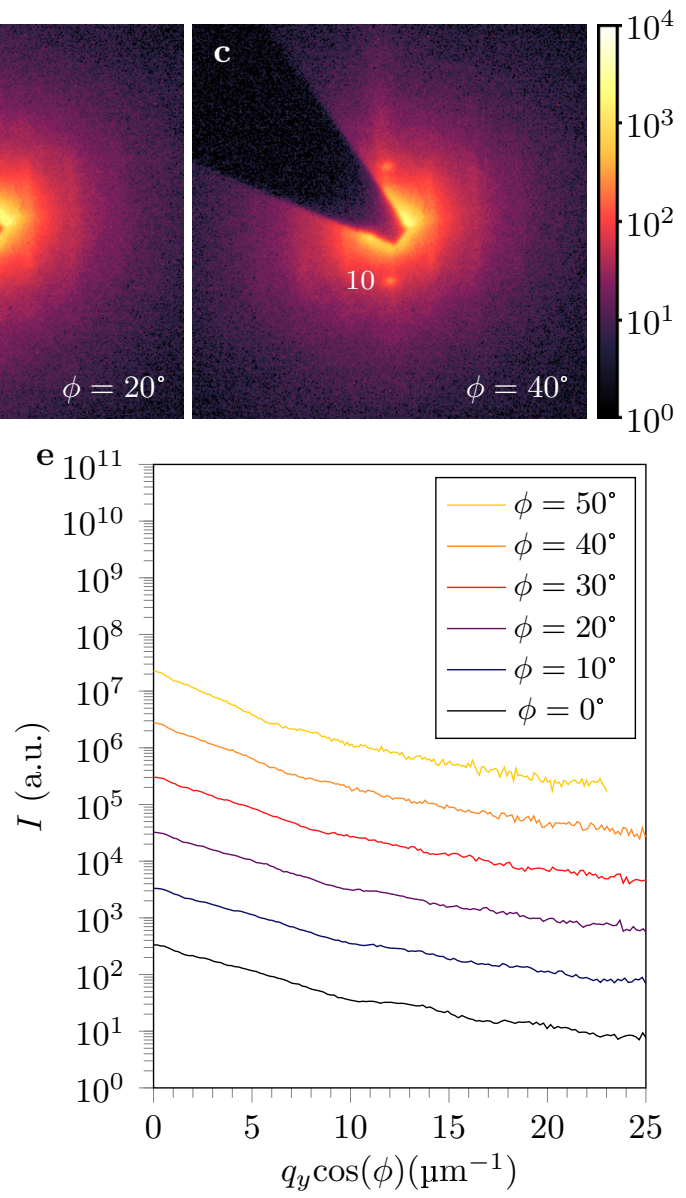

Figure 3.4: SAXS diffraction patterns of a monolayer prepared from colloidal silica superballs with an average diameter of $1.01 \mu \mathrm{m} \pm 6.6 \%$ and an average $m$-value of $3.63 \pm 12 \%$ imaged at rotations around the $x$-axis of $0^{\circ}(\mathrm{a}), 20^{\circ}(\mathrm{b})$ and $40^{\circ}(\mathrm{c})$. Cross sections of the diffraction patterns at $q_{x}=0 \mu \mathrm{m}^{-1}$ (d) and at $q_{x}=5.76 \mu \mathrm{m}^{-1}$ (e). The positions of the identified peaks are visualized in the cross sections at the corresponding $q_{x} \cos (\phi)$ positions.

We have numerically calculated the form factors of the three superballshaped colloids discussed in this chapter to further understand the visibility and appearance and disappearance of the various Bragg peaks. The numerically calculated form factors of the spherical, semi-cubic and cube-like superballs are depicted in figure $3.5 \mathrm{a}, \mathrm{b}$ and $\mathrm{c}$ respectively. While the form factor of the spherical superballs can be calculated analytically, as shown earlier in figure $3.2 \mathrm{e}$, it is included here as a numerical calculation for comparison. In these images the scale of the calculated intensity is limited to display only 4 orders of magnitude for clarity as this is approximately equal to the range of the detector used in the experiments. It can be seen that, upon increasing the 
shape parameter $m$ of the colloidal superballs, the concentric rings observed in the form factor of the colloidal spheres begin to show oscillations with maxima in their intensity near the flat sides of the superballs, while their intensity decreases in the diagonal directions. As was previously shown by Meijer et al. [10], the form factor of cube-like superballs have cross-like shapes with little intensity in the corners of the diffraction patterns. This results in peaks in these locations to be less visible which further complicates the analysis. This can also clearly be seen in the scattering pattern of the cube-like superballs, depicted in figure $3.4 \mathrm{a}$, where little intensity can be observed in the corners of the image.
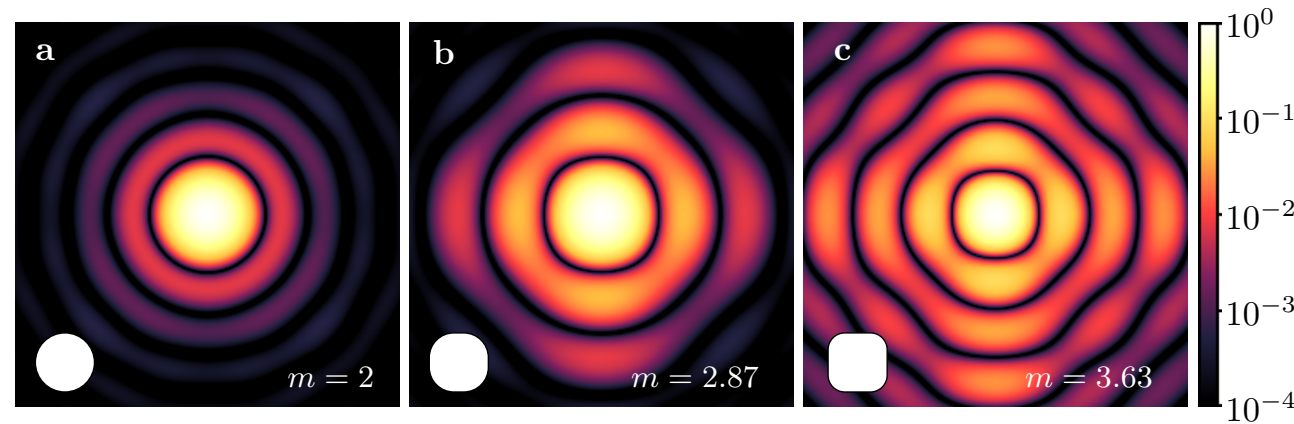

Figure 3.5: Numerically calculated form factors of the spherical (a), semi-cubic (b) and cubic (b) superballs. The orientation of the superball is depicted in the insets of the corresponding images. The intensity is limited to 4 orders of magnitude, approximately equal to the range of the detector used in these experiments.

Now that we have assigned all identifiable Bragg peaks in the diffraction patterns of the spherical, semi-cubic and cube-like superballs, we can begin to deduce their structure factors $S(\vec{q})$ and their lattice structures in real space. We have observed that the Bragg peaks in the horizontal direction become increasingly elongated in the vertical $y$-direction upon increasing the shape parameter $m$ of the superball particles and eventually form apparent continuous bands for the cube-like superballs. The Bragg peaks do not, however, broaden in the horizontal direction but remain sharp and well-defined upon increasing the shape parameter $m$ of the superballs. The deduced structure factors for the spherical, semi-cubic and cube-like superballs along with the identified Bragg peaks and their reciprocal basis lattice vectors are schematically depicted in figure $3.6 \mathrm{a}, \mathrm{b}$ and $\mathrm{c}$ respectively. Here, the length of the first primitive reciprocal basis vector $\vec{b}_{1}$ can be calculated from the positions of the 10, 20 and 30 peaks according to equation 3.5. For the spherical and semicubic superballs, we can determine the length of the second reciprocal basis vector $\vec{b}_{2}$ from the positions of the 01,11 and 21 peaks. Since for the cube-like superballs the $01,11,21$ appear to form a single continuous band, we instead define $\vec{b}_{2 x}$ as the horizontal component of $\vec{b}_{2}$ so that $\vec{b}_{2 x}$ is perpendicular to 
$\vec{b}_{1}$. From the reciprocal basis lattice vectors $\vec{b}_{1}$ and $\vec{b}_{2}$, we can determine the real space primitive lattice vectors $\vec{a}_{1}$ and $\vec{a}_{2}$ and, in the case of the cube-like superballs, $\vec{a}_{2 x}$. Alternatively, their lengths, $a_{1}$ and $a_{2}$, can also be calculated using equation 3.7 .
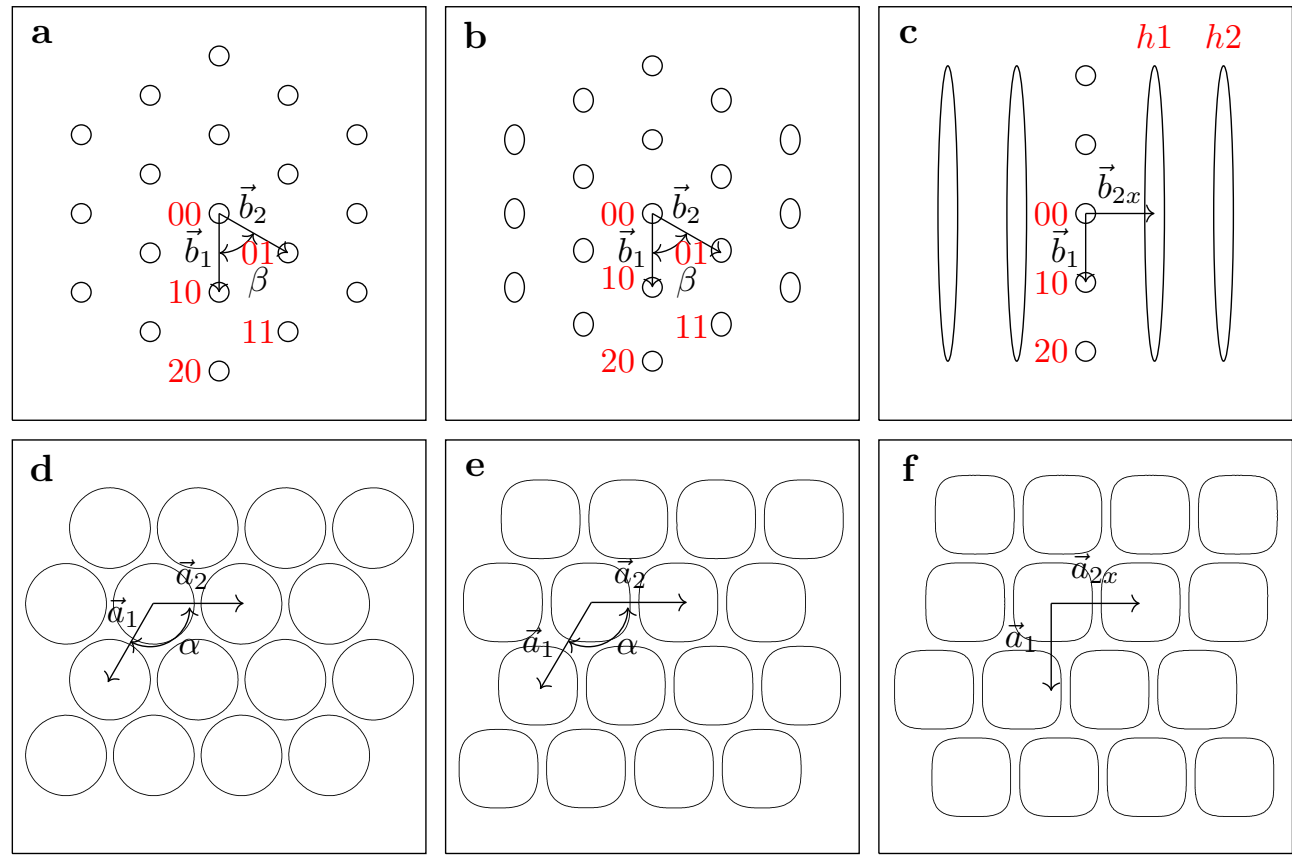

Figure 3.6: The deduced structure factor $P(\vec{q})$ in reciprocal space (top row) and the corresponding lattice structures in real space (bottom row) for the solid spherical (a and d), semi-cubic (b and e) and cube-like (c and f) superballs. Here $\vec{b}_{1}$ and $\vec{b}_{2}$ are the reciprocal basis lattice vectors, separated by angle $\beta$, and $\vec{a}_{1}$ and $\vec{a}_{2}$, separated by angle $\alpha$, are the corresponding basis lattice vectors. For the cube-like superballs we have instead defined $\vec{b}_{2 x}$ as the horizontal component that is perpendicular to $\vec{b}_{1}$. The Bragg peaks are labelled on their left. The direction of the mechanical rubbing is along the horizontal direction.

The corresponding real space lattices of the spherical, semi-cubic and cubelike superballs along with their primitive lattice vectors are schematically depicted in figure $3.6 \mathrm{~d}$, e and $\mathrm{f}$. For the spherical superballs we determined the length of the primitive lattice vectors to be $a_{1}=1.21 \mu \mathrm{m}$ and $a_{2}=1.19 \mu \mathrm{m}$, with an angle of $\alpha=119.4^{\circ}$. This confirms that these superballs indeed form a hexagonal lattice in real space with a well-defined and equal periodicity in all directions. For the semi-cubic superballs we find that $a_{1}=1.11 \mu \mathrm{m}$ and $a_{2}=1.04 \mu \mathrm{m}$, separated by an angle of $\alpha=117.8^{\circ}$. An angle of $\alpha<120^{\circ}$ suggest that these superballs form a rhombic lattice instead as expected for the densest packing of superdisks in two dimensions [14]. From $a_{2}<a_{1}$ we can 
further conclude that these semi-cubic superballs are oriented with their flat faces in the horizontal and vertical direction. For the cube-like superballs that form a more cube-like lattice we found that $a_{1}=1.05 \mu \mathrm{m}$ and $a_{2 x}=1.08 \mu \mathrm{m}$. Due to the broadening of the Bragg peaks in the vertical direction upon increasing the shape parameter $m$ of the superballs, it can be concluded that these colloidal superballs have formed chains in the horizontal direction which are able to slide inceasingly freely with respect to each other upon an increase in the shape parameter $m$. However, the exact degree of sliding cannot be determined solely with SAXS.

\subsection{Conclusion}

Monolayers of spherical, semi-cubic and cube-like superballs were prepared using the unidirectional rubbing method and analysed with SAXS. Using these three shapes of superballs we have shown that some Bragg peaks may not appear in the diffraction patterns due to the presence of minima in their form factor. Due to the anisotropic shape and the hollow nature of the more cubicshaped superballs it is difficult to predict which Bragg peaks are missing, which complicates the analysis. By imaging the colloidal monolayers under a range of rotations around the $x$-axis the $\vec{q}$-values of the Bragg peaks can be shifted away from the minima in the form factor. This can visualize the Bragg peaks which might otherwise not be visible with the sample oriented perpendicular to the incoming X-ray beam. Thus, by exploring the three-dimensional reciprocal space of a two-dimensional sample we are able to analyse the structure of the colloidal monolayer in greater detail.

For all three superballs shapes, ranging from spherical to almost cubic, well-defined periodic structures resulted upon their assembly into monolayers using the unidirectional rubbing method. It was observed that the spherical superballs formed hexagonal lattices with a high degree of positional order in all directions. Upon increasing the shape parameter $m$ of the superballs it was observed that this high degree of positional order in the direction of rubbing remained, but weakened in the direction perpendicular to the direction of rubbing. All three superballs formed well-defined chains in the direction of rubbing as evidenced by the sharp Bragg peaks in the diffraction pattern along the vertical direction. For the semi-cubic superballs with a shape parameter of $m=2.87$ these chains were able to shift slightly with respect to each other but still displayed a preference for a rhombic crystal lattice structure. For the cube-like superballs with a shape parameter of $m=3.63$, however, these chains were able to slide much more freely with respect to each other, as evidenced by the apparent continuous bands present in the diffraction patterns. The exact degree of sliding present, however, could not be determined with SAXS alone.

We therefore conclude that SAXS is a very powerful method for studying 
the structures of these monolayers of colloidal silica superballs, but that it has its limitations. In the following chapter we continue to study these monolayers of colloidal silica superballs prepared using the unidirectional rubbing method and use scanning electron microscopy (SEM) in addition to SAXS to study the effect of size and shape on their assembly.

\subsection{Acknowledgements}

Janne-Mieke Meijer is thanked for the fruitful discussions, her help with the analysis of the data and for checking the writing. Dominique Thies-Weesie is thanked for her help with the preparation of the spherical silica colloids. Nicholas Orr is thanked for his help at the beamline of the ESRF. Michela Brunelli and Daniel Hermida Merino are thanked for providing technical support at the beamline. The Nederlandse Organisatie voor Wetenschappelijk Onderzoek (NWO) is acknowledged for providing the SAXS beamtime.

\section{References}

[1] M. A. Boles, M. Engel, and D. V. Talapin, "Self-assembly of colloidal nanocrystals: From intricate structures to functional materials," Chemical Reviews, vol. 116, no. 18, pp. $11220-11289,2016$.

[2] Z. Xu, L. Wang, F. Fang, Y. Fu, and Z. Yin, "A review on colloidal self-assembly and their applications," Current Nanoscience, vol. 12, no. 6, pp. 725-746, 2016.

[3] Q. Chen, S. C. Bae, and S. Granick, "Directed self-assembly of a colloidal kagome lattice," Nature, vol. 469, no. 7330, pp. 381-384, 2011.

[4] J. Yan, M. Han, J. Zhang, C. Xu, E. Luijten, and S. Granick, "Reconfiguring active particles by electrostatic imbalance," Nature Materials, vol. 15, no. 10, pp. 1095-1099, 2016.

[5] J. Zhang, B. A. Grzybowski, and S. Granick, "Janus particle synthesis, assembly, and application," Langmuir, vol. 33, no. 28, pp. 6964-6977, 2017.

[6] C. Park, T. Lee, Y. Xia, T. J. Shin, J. Myoung, and U. Jeong, "Quick, large-area assembly of a single-crystal monolayer of spherical particles by unidirectional rubbing," Advanced Materials, vol. 26, no. 27, pp. 4633-4638, 2014.

[7] C. Park, K. Koh, and U. Jeong, "Structural Color Painting by Rubbing Particle Powder," Scientific Reports, vol. 5, pp. 1-5, 2015.

[8] L. Cademartiri, K. J. Bishop, P. W. Snyder, and G. A. Ozin, "Using shape for self-assembly," Philosophical Transactions of the Royal Society A: Mathematical, Physical and Engineering Sciences, vol. 370, no. 1969, pp. 2824-2847, 2012. 
[9] J. M. Meijer, D. V. Byelov, L. Rossi, A. Snigirev, I. Snigireva, A. P. Philipse, and A. V. Petukhov, "Self-assembly of colloidal hematite cubes: A microradian Xray diffraction exploration of sedimentary crystals," Soft Matter, vol. 9, no. 45, pp. 10729-10 738, 2013.

[10] J. M. Meijer, A. Pal, S. Ouhajji, H. N. Lekkerkerker, A. P. Philipse, and A. V. Petukhov, "Observation of solid-solid transitions in 3D crystals of colloidal superballs," Nature Communications, vol. 8, pp. 1-8, 2017.

[11] J. M. Meijer, V. Meester, F. Hagemans, H. N. Lekkerkerker, A. P. Philipse, and A. V. Petukhov, "Convectively assembled monolayers of colloidal cubes: Evidence of optimal packings," Langmuir, vol. 35, no. 14, pp. 4946-4955, 2019.

[12] J. M. Meijer, F. Hagemans, L. Rossi, D. V. Byelov, S. I. Castillo, A. Snigirev, I. Snigireva, A. P. Philipse, and A. V. Petukhov, "Self-assembly of colloidal cubes via vertical deposition," Langmuir, vol. 28, no. 20, pp. 7631-7638, 2012.

[13] A. V. Petukhov, J. M. Meijer, and G. J. Vroege, "Particle shape effects in colloidal crystals and colloidal liquid crystals: Small-angle X-ray scattering studies with microradian resolution," Current Opinion in Colloid and Interface Science, vol. 20, no. 4, pp. 272-281, 2015.

[14] Y. Jiao, F. H. Stillinger, and S. Torquato, "Optimal packings of superdisks and the role of symmetry," Physical Review Letters, vol. 100, no. 24, pp. 2-5, 2008. 



\section{Chapter 4}

\section{Mechanical Assembly of Colloidal Superball Monolayers}

\section{Abstract}

We have prepared monolayers of colloidal superballs with various shapes and sizes using the unidirectional rubbing method to determine the influence of the superball shape and size on the resulting lattice structures, as well as the influence of the rubbing speed and pressure at which they are prepared. Using this assembly method the dried colloidal superballs were mechanically rubbed onto a polydimethylsiloxane- (PDMS) coated surface using a custom-made rubbing device. We have used both scanning electron microscopy (SEM) and small angle X-ray scattering (SAXS) to study their lattice structures in detail. Well-defined lattice structures were observed over a broad range of rubbing pressures and speeds, with a continuous transition between the hexagonal lattice for spheres and the so-called sliding phase for cube-like superballs. Using SEM to study the local ordering of the superballs we have found that in the lattices of cube-like superballs, with shape parameters of around $m=3.5$ and up, the chains assembled along the rubbing direction were able to slide completely free with respect to each other with no significant preferential alignment. 


\subsection{Introduction}

The shape of colloidal particles is well-known to influence their self-assembled structures [1-4]. Anisotropic colloids have been shown to be able to selfassemble into a wide variety of periodic structures depending on their shape. As we have already described in the previous chapters, the superball shape is an interpolation between the spherical and the cubic shape and this variable shape allows smooth phase transitions to occur. Crystals of colloidal superballs have been prepared in both two and three dimensions using a wide variety of methods with various driving forces for their self-assembly $[5,6]$. For example, the addition of both polymer and colloidal depletants has been shown to result in various lattice structures, ranging from the cubic-lattice to the $\Lambda_{0^{-}}$and $\Lambda_{1}$-lattices depending on the shape of the colloidal superballs and their size relative to the size of the added depletant $[7,8]$. Furthermore, the influence of the superball shape on the resulting lattices has been studied using both the horizontal and vertical deposition method where mainly a mixture of the $\Lambda_{0^{-}}$ and $\Lambda_{1}$-lattices were observed, where their ratio of occurrence was a function of the superball shape $[9,10]$. These observations are in agreement with molecular dynamics simulations where it was found that the $\Lambda_{0}$-lattice is only very slightly denser than the $\Lambda_{1}$-lattice for superballs with a more cube-like shape [11].

A novel method for the assembly of colloids into monolayers is the recently developed unidirectional rubbing method where a mechanical force is used to rub the dried colloids between two sticky surfaces to assemble them into twodimensional colloidal lattices $[12,13]$. Here, well-defined hexagonal lattices were observed to form from colloidal spheres over a wide range of rubbing speeds and pressures, which makes it a promising method for the preparation of colloidal monolayers. Furthermore, this method is easy to apply and does not rely on specialized knowledge or expensive materials and equipment and should therefore be accessible to a broad audience.

In the previous chapter we have presented a strategy for the measurement and analysis of monolayers of colloidal superballs prepared using the unidirectional rubbing method with small-angle X-ray scattering (SAXS). Briefly, by imaging the colloidal superball monolayers at various rotations around the $x$ axis, the $\vec{q}$-values of the Bragg peaks in the diffraction patterns can be shifted. This shift allows the Bragg peaks which coincident with the minima present in the form factor to be visualized, resulting in a more accurate and complete analysis of the samples. Here, we have already seen that the unidirectional rubbing method can be used to assemble colloidal superballs into well-defined two-dimensional lattices. A transition in the resulting lattices was observed upon increasing the shape parameter $m$ of the superballs, ranging from the hexagonal lattice for spherical superballs to the rhombic lattice for semi-cubic superballs to the so-called sliding phase for cube-like superballs. In the sliding 
phase, chains of superballs form in the direction of the applied rubbing and appear to become increasingly able to slide freely with respect to each other upon increasing their shape parameter $m$. The exact degree of sliding could not be determined with SAXS alone, however.

In this chapter we continue studying the lattice structures in monolayers of colloidal superballs prepared using the unidirectional rubbing method. In addition to SAXS, we employ scanning electron microscopy (SEM) to study the local ordering of the obtained colloidal superball lattices. We first consider the influence of the rubbing speed and pressure on the lattice structures of monolayers of spherical superballs which we then compare with those obtained from more cubic superballs. Using a fixed and suitable combination of a rubbing speed and pressure we then determine the effect of the shape and size of the superballs on the resulting two-dimensional lattice structures for a wide variety of superball shapes and sizes. Using these two complementary analysis techniques we study the continuous phase transition from the hexagonal lattice for spheres to the sliding phase for the cube-like superballs.

\subsection{Experimental methods}

\subsubsection{Preparation of the colloidal superball monolayers}

The solid silica spheres were prepared using the Stöber process as described in subsection 2.1.1. The hollow silica superballs were prepared by coating hematite cubes with a layer of silica using a modified Stöber process and subsequently dissolving the hematite core as shown in subsection 2.1.2. Here, the colloidal superballs with a relatively low shape parameter $m$ were prepared by repeating the silica coating step up to three times. The average shape parameter $m$ and edge diameter $D$ of the resulting superball colloids were determined using a custom-written Python script from their transmission electron microscopy (TEM) images as described in subsection 2.1.3. The colloidal silica superballs with various shapes and sizes were assembled into monolayers by mechanically rubbing them onto polydimethylsiloxane- (PDMS) coated glass slides (\#0, $60 \mathrm{~mm} \times 24 \mathrm{~mm} \times 0.085-0.12 \mathrm{~mm}$ ) using the custom-made device presented in section 2.4. For all samples the device was set to run 10 rubbing cycles in order to prepare the colloidal superball monolayers.

\subsubsection{Analysis of the colloidal superball monolayers using SAXS}

The experimental details of the SAXS setup are described in subsection 3.3.2. The analysis strategy of the diffraction patterns and the determination of the lengths of the primitive reciprocal basis vectors $\vec{b}_{1}$ and $\vec{b}_{2}$ and the corresponding lengths of the primitive lattice vectors $\vec{a}_{1}$ and $\vec{a}_{2}$ are described in section 3.3 . 


\subsubsection{Analysis of the colloidal superball monolayers using SEM}

The colloidal superball monolayers were imaged with SEM using a Phenom Pro X scanning electron microscope with an electron energy of $10 \mathrm{kV}$. The SEM samples were prepared by cutting pieces of several $\mathrm{cm}^{2}$ in size from the center of the glass slides containing the colloidal superball monolayers. These pieces were attached to a specimen stub using double-sided and electrically conductive tape and subsequently sputter coated with a $10 \mathrm{~nm}$ thick layer of platinum using a Cressington 208HR sputter coater. The conductive platinum layer was found to increase the contrast of the resulting images and prevented the accumulation of negative charge which may decrease the resolution of the images. All samples were imaged with the direction of the mechanical rubbing along the horizontal direction. For each of the samples at least 20 SEM images were collected from various regions of the colloidal monolayers in order to obtain representative SEM images.

The resulting SEM images were analysed using a custom written Python script. The analysis process is illustrated in figure 4.1. First, an adaptive threshold was applied in order to binarize the images as depicted in figure $4.1 \mathrm{a} \rightarrow \mathrm{b}$. The outlines of the particles were then filled to obtain the shape of the particles as depicted in figure $4.1 \mathrm{~b} \rightarrow \mathrm{c}$. Next, up to 5 erosion cycles were performed in order to separate any connected particles in the binarized images as depicted in figure $4.1 \mathrm{c} \rightarrow \mathrm{d}$. Care was taken to not apply more erosion cycles than necessary as this may increase the errors in the position and the orientation of particles. From the resulting images the center of mass of the particles were then determined as the average position of their pixels in the $x, y$-plane. The center of mass of the particles are displayed in figure $4.1 \mathrm{~d}$ as red dots.

To determine the orientation of the non-spherical superballs, the angle of each pixel of the particle with respect to the particles center of mass was first calculated and distributed over 120 bins. Each corner of the particle forms a maximum in the histogram which plots the frequency of pixels with a certain orientation of $\theta$ in the range of $0 \leq \theta<2 \pi$. An example of these plots is depicted in figure 4.1f. Since the non-spherical superballs have four-fold symmetry in two dimensions, we can subtract angles of $\pi / 2$ from all values of $\theta$ until they fall in the range in the range of $0 \leq \theta^{\prime}<\pi / 2$ as depicted in figure $4.1 \mathrm{~g}$. The bin with the highest frequency is then determined to be the orientation of one of the corners of the non-spherical superballs. Finally, a value of $\frac{\pi}{4}$ is subtracted from $\theta^{\prime}$ so that the angle of the superball particles ranges from $-\frac{\pi}{4} \leq \theta "<\frac{\pi}{4}$, where superballs with an angle of $\theta "=0$ are aligned with their faces in the horizontal direction, the direction in which the mechanical rubbing was applied. The determined orientations of the superball particles in this example are displayed in figure 4.1e. 

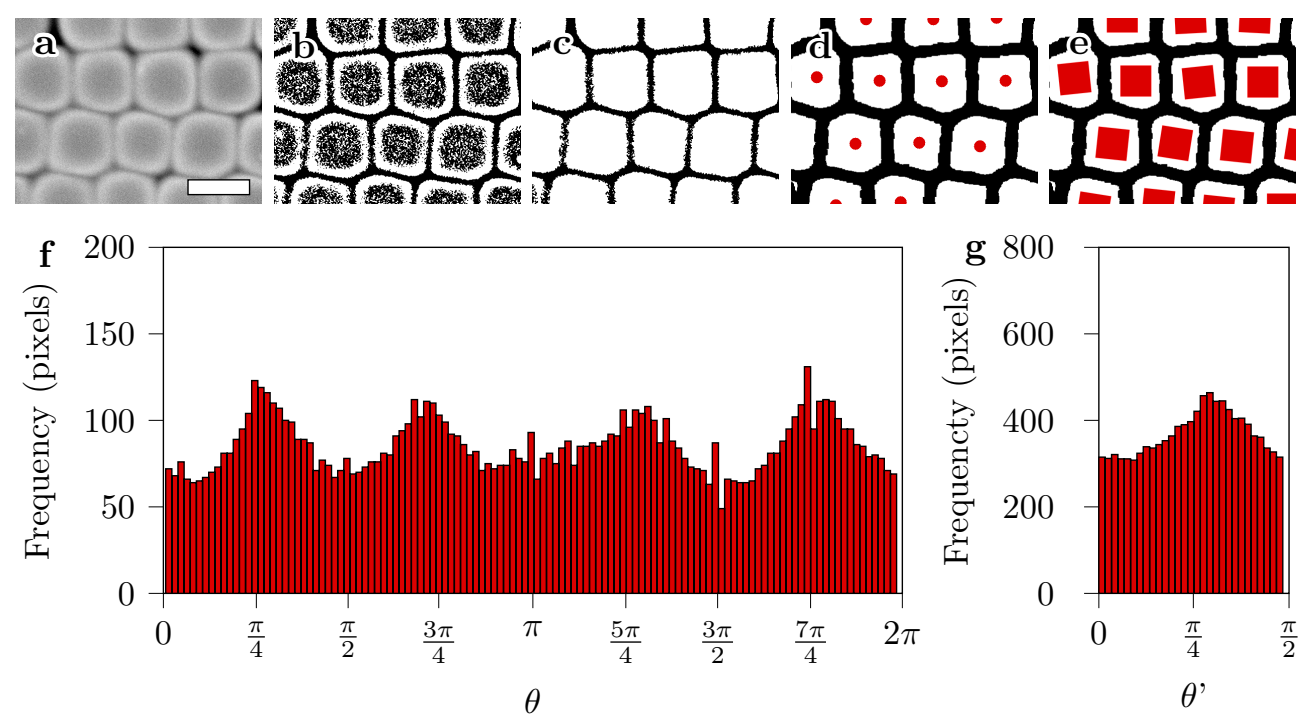

Figure 4.1: An example of the image processing of the SEM images and the tracking of the particles. First, an adaptive threshold was applied to binarize the SEM images $(\mathrm{a} \rightarrow \mathrm{b})$. The outlines of the particles were then filled $(\mathrm{b} \rightarrow \mathrm{c})$ and up to 5 erosion cycles were performed to separate any connected particles $(c \rightarrow d)$. The center of mass of the particles were determined as the average position of all pixels of the particles $(d)$. The angle of each pixel with respect to the position of the particles center of mass are then distributed over 120 bins (f). The four-fold multiplicity is then removed by subtracting $\pi / 2$ from all values of $\theta$ until they fall in the range in the range of $0 \leq \theta^{\prime}<\pi / 2(\mathrm{~g})$. The bin with the highest frequency is then determined to be the orientation of one of the corners of the non-spherical superballs. The determined orientations of the particles in this example are displayed in (e). The scale bar in (a) represents $1 \mu \mathrm{m}$.

In the example shown in figure 4.1 it can be seen that these relatively cube-like superballs may appear as pentagons or hexagons in the binarized images depending on the relative positions of its surrounding particles. We have found this analysis method to be the most reliable method to determine the orientation of the colloidal particles as any distortions in the shape of the particle in the binarized image are not averaged into the calculation but are instead largely ignored as a shoulder in the histogram.

From the positions of the particles center of mass in $x, y$-space we can calculate the two-dimensional pair distribution function, which visualizes the local density plot of the neighbouring particles' positions. Of the particle pairs one particle is taken as the origin so that the location of the other particle describes the probability of finding a particle at a specific position in $x, y$-space. Since the unidirectional rubbing method aligns all colloidal crystal grains in the direction of rubbing, the orientation of the superballs is not taken into account in the calculation of the two-dimensional pair distribution function. 


\subsection{Results and discussion}

\subsubsection{Influence of the rubbing speed and pressure}

Monolayers of both spherical and cube-like superballs were prepared at various rubbing speeds $v_{r}$ and pressures $p_{r}$ in order to determine how these parameters influence the resulting lattice structures. We first consider spherical superballs with an average diameter of $D=1.25 \mu \mathrm{m} \pm 3.7 \%$. Representative SEM images of three examples of monolayers prepared from these spheres are depicted in figure $4.2 \mathrm{a}, \mathrm{b}$ and $\mathrm{c}$. These three monolayers were prepared at rubbing speeds and pressures of $v_{r}=1 \mathrm{~mm} / \mathrm{s}$ and $p_{r}=50 \mathrm{~g}, v_{r}=5 \mathrm{~mm} / \mathrm{s}$ and $p_{r}=50 \mathrm{~g}$ and $v_{r}=5 \mathrm{~mm} / \mathrm{s}$ and $p_{r}=400 \mathrm{~g}$ respectively. In all three SEM images depicted in figure $4.2 \mathrm{a}, \mathrm{b}$ and $\mathrm{c}$ well-defined hexagonal close-packed lattices of the colloidal spheres can be seen with a strong alignment of the colloidal crystal grains in the horizontal direction which is the direction in which the mechanical rubbing was applied.

Using SAXS the long-range order of the lattice structures can be studied in detail with good statistics. The corresponding SAXS diffraction patterns imaged at a rotation around the $x$-axis of $0^{\circ}$, thus with the sample placed perpendicular to the incoming X-ray beam, are depicted in figure $4.2 \mathrm{~d}$, e and $\mathrm{f}$ respectively. Here, the presence of sharp Bragg peaks with six-fold symmetry observed in all three diffraction patterns confirm that the colloidal spheres are arranged in well-defined hexagonal close-packed lattices for all three samples. The sharpness of the Bragg peaks indicate that these lattices have a high periodicity in all directions and that the lattices are indeed strongly aligned in the direction of rubbing.

As explained in the previous chapter, these hexagonal lattices can be characterized quantitatively with SAXS using the primitive lattice vectors $\vec{a}_{1}$ and $\vec{a}_{2}$. An overview of the identifiable Bragg peaks and the primitive reciprocal basis vectors in the diffraction patterns along with the primitive lattice vectors in the corresponding real space lattice structures are depicted in figure 3.6 on page 46 . Here, for the cube-like superballs however, we have instead defined $\vec{b}_{2 x}$ as the horizontal component of $\vec{b}_{2}$ as the latter cannot be defined as a result of the formation of continuous $h 1$ and $h 2$ bands. To allow for the lattices of spherical and more cube-like superballs to be conveniently compared later, we shall use $\vec{b}_{2 x}$ and $\vec{a}_{2 x}$ for all superball shapes in the remainder of this chapter. Furthermore, to make these primitive lattice vectors independent of the superball size, we shall normalize them with the edge diameter $D$ of the superballs determined using TEM. The resulting $a_{2 x} / D$ describes the normalized horizontal interparticle distance between adjacent particles in the chains formed in the horizontal direction while $a_{1} / D$ describes the normalized vertical distance between adjacent chains of particles. For each of the samples the lengths of $a_{1}$ and $a_{2 x}$ were determined from 8 diffraction patterns imaged 
at various locations of the sample to ensure proper statistics and to allow for their standard deviations to be determined. The determined values of $a_{1} / D$ and $a_{2 x} / D$, along with their standard deviations, are depicted in figure $4.2 \mathrm{~g}$ and $\mathrm{h}$ respectively for all combinations of rubbing speeds of $v_{r}=1,3$ and $5 \mathrm{~mm} / \mathrm{s}$ and pressures of $p_{r}=50,100,200$ and $400 \mathrm{~g}$.
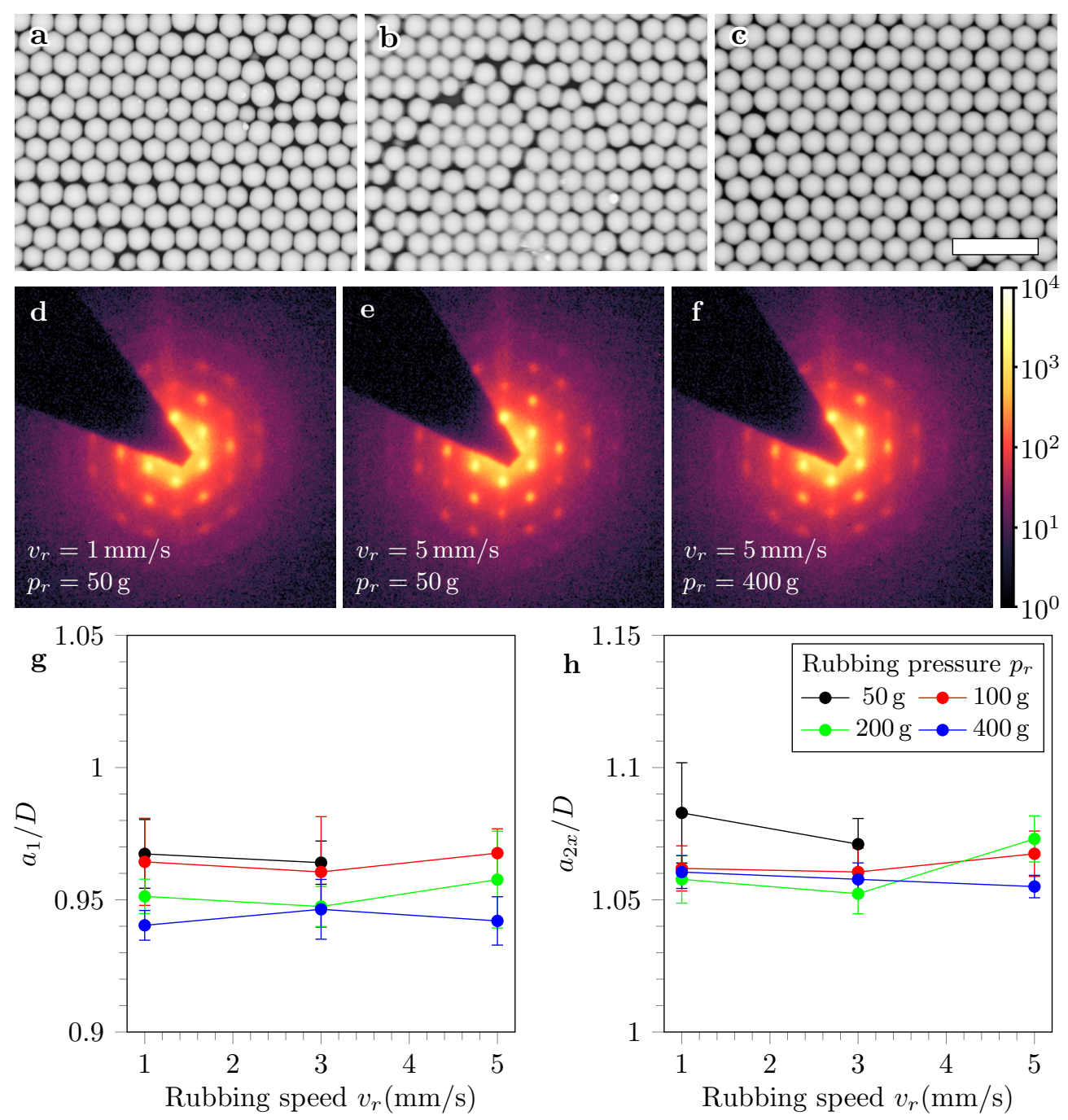

Figure 4.2: Representative SEM images and SAXS diffraction patterns of monolayers of colloidal silica spheres with an average diameter of $D=1.25 \mu \mathrm{m} \pm 3.7 \%$ prepared using the unidirectional rubbing method at combinations of rubbing speeds and pressures of $v_{r}=1 \mathrm{~mm} / \mathrm{s}$ and $p_{r}=50 \mathrm{~g}$ (a and d), $v_{r}=5 \mathrm{~mm} / \mathrm{s}$ and $p_{r}=50 \mathrm{~g}$ (b and e) and $v_{r}=5 \mathrm{~mm} / \mathrm{s}$ and $p_{r}=400 \mathrm{~g}$ (c and $\mathrm{f}$ ). The diffraction patterns were imaged at a rotation around the $x$-axis of $\phi=0^{\circ}$. The determined normalized lattice vectors $a_{1} / D(\mathrm{~d})$ and $a_{2 x} / D(\mathrm{e})$ are shown for all combinations of rubbing speeds $\left(v_{r}\right)$ and pressures $\left(p_{r}\right)$. The scale bar represents $5 \mu \mathrm{m}$. 
In figure $4.2 \mathrm{~g}$ and $\mathrm{h}$ it can be seen that both $a_{1} / D$ and $a_{2 x} / D$ decrease significantly upon increasing the rubbing pressure $p_{r}$, especially from a rubbing pressure of $p_{r}=50$ and 100 to 200 and $400 \mathrm{~g}$. A higher rubbing pressure thus results in a more densely packed lattice structure. This is a plausible result as the particles are pushed together more strongly during the assembly process upon increasing the rubbing pressure. In addition, it can be seen that the standard deviation in both $a_{1} / D$ and $a_{2 x} / D$ decreases upon increasing the rubbing pressure $p_{r}$. No clear trends, however, can be seen in $a_{1} / D$ and $a_{2 x} / D$ upon increasing the rubbing speed $v_{r}$. The small variations in the values of both $a_{1} / D$ and $a_{2 x} / d$ observed from sample to sample can be ascribed to experimental imperfections such as, for example, an uneven distribution of the dried colloids on the PDMS-coated glass sliding during preparation or differences in the grain size of the dried colloids. These experimental parameters, however, are difficult to control. It can be concluded that for the colloidal spheres the influence of the rubbing pressure on the resulting structures is very small as the ratio of $a_{1} / D$ and $a_{2 x} / D$ only varies a few percent upon a variation in the rubbing pressure of almost an entire order of magnitude. In addition, the angle $\alpha$ between $\vec{a}_{1}$ and $\vec{a}_{2}$ falls between $119^{\circ}$ and $120^{\circ}$ for all samples and thus does not deviate significantly from $\alpha=120^{\circ}$ for an ideal hexagonal close-packed lattice. Similar to the known work on the unidirectional rubbing method, we find that well-defined hexagonal close-packed lattices can be formed from colloidal spheres in a wide range of rubbing speeds and pressures [12].

Similarly, monolayers of cube-like superballs with an average shape parameter of $m=3.40 \pm 11 \%$ and edge diameter of $D=1.28 \mu \mathrm{m} \pm 4.4 \%$ were prepared at all combinations of rubbing speeds of $v_{r}=1,3$ and $5 \mathrm{~mm} / \mathrm{s}$ and pressures of $p_{r}=50,100,200$ and $400 \mathrm{~g}$. Representative SEM images of three examples of monolayers prepared from these cube-like superballs are depicted in figure $4.3 \mathrm{a}, \mathrm{b}$ and $\mathrm{c}$. In figure $4.3 \mathrm{a}$, where the colloidal superball monolayer was prepared using a rubbing speed of $v_{r}=1 \mathrm{~mm} / \mathrm{s}$ and pressure of $p_{r}=50 \mathrm{~g}$, it can be seen that these superballs did not form a well-defined lattice. However, the superballs appear to have a very similar orientation with all their flat faces oriented towards to the PDMS-coated substrate and aligned in the horizontal direction, the direction of rubbing. In figure $4.3 \mathrm{~b}$ it can be seen that, upon increasing the rubbing pressure to $p_{r}=400 \mathrm{~g}$ while keeping the rubbing speed at $v_{r}=1 \mathrm{~mm} / \mathrm{s}$, the lattice is much better defined, more densely packed and has a stronger alignment in the direction of rubbing. Upon increasing the rubbing speed to $v_{r}=5 \mathrm{~mm} / \mathrm{s}$, as depicted in figure $4.3 \mathrm{c}$, no significant difference in the lattice structures can be observed by eye. The corresponding SAXS diffraction patterns imaged at a rotation around the $x$-axis of $\phi=0^{\circ}$ are depicted in figure 4.3d, e and f respectively. Here, the presence of broad but separate Bragg peaks in the diffraction pattern depicted in figure $4.3 \mathrm{~d}$ indicates that the colloidal superballs had formed a lattice structure but with a periodicity that is not very well-defined at the relatively low rubbing speed and pressure of $v_{r}=1 \mathrm{~mm} / \mathrm{s}$ and $v_{p}=50 \mathrm{~g}$ respectively. At a high rubbing 
pressure of $p_{r}=400 \mathrm{~g}$ the peaks in the diffraction patterns became much sharper, indicating the formation of a crystal lattice with a more well-defined periodicity. By eye the diffraction pattern does not appear to change upon increasing the rubbing speed to $v_{r}=5 \mathrm{~mm} / \mathrm{s}$ as depicted in figure $4.3 \mathrm{f}$.
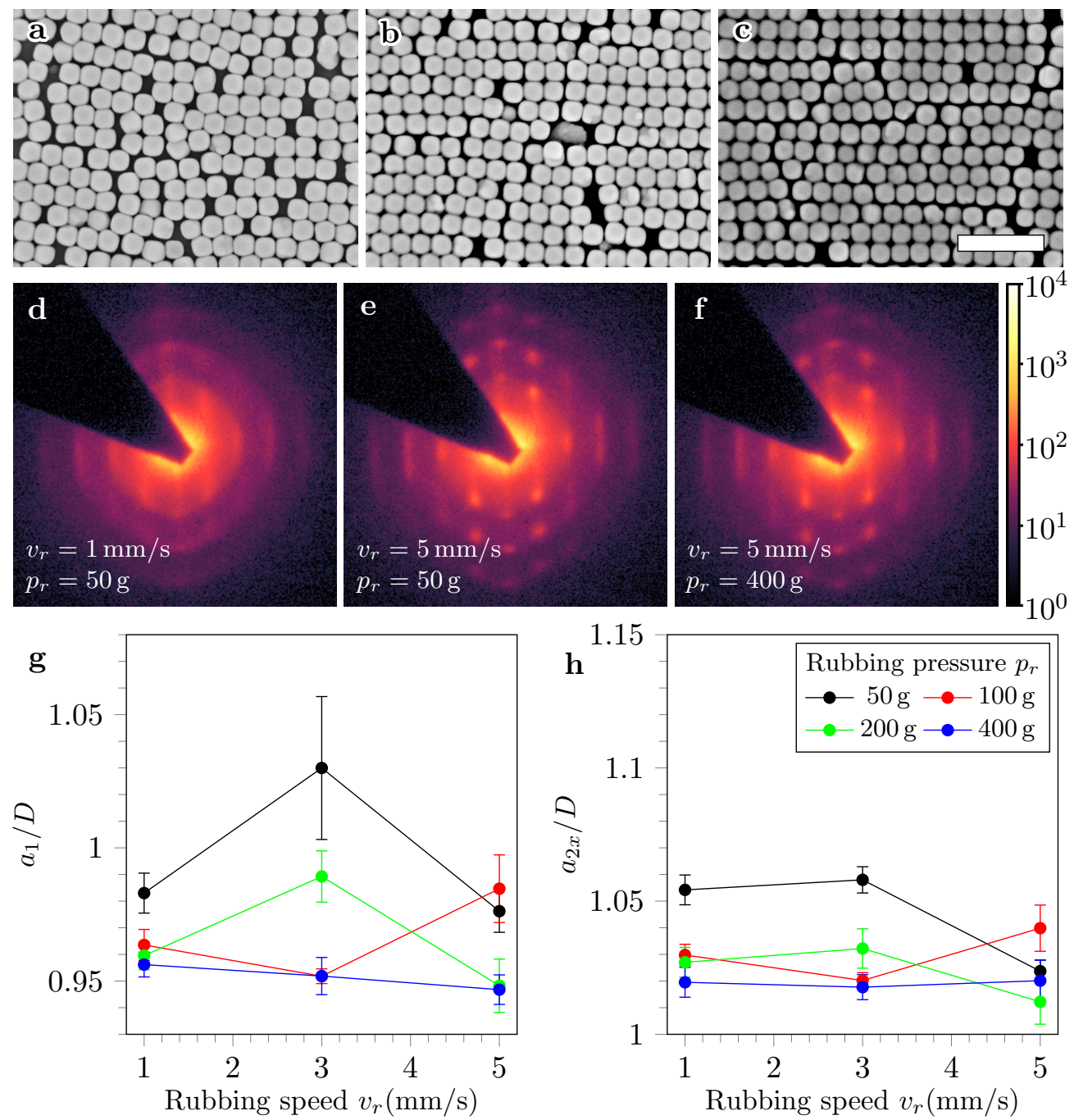

Figure 4.3: Representative SEM images and SAXS diffraction patterns of monolayers of colloidal cube-like silica superballs with an average diameter of $D=1.26 \mu \mathrm{m}$ $\pm 3.7 \%$ and shape parameter of $m=3.40$ prepared using the unidirectional rubbing method at combinations of rubbing speeds and pressures of $v_{r}=1 \mathrm{~mm} / \mathrm{s}$ and $p_{r}=50 \mathrm{~g}(\mathrm{a}$ and $\mathrm{d}), v_{r}=5 \mathrm{~mm} / \mathrm{s}$ and $p_{r}=50 \mathrm{~g}(\mathrm{~b}$ and $\mathrm{e})$ and $v_{r}=5 \mathrm{~mm} / \mathrm{s}$ and $p_{r}=400 \mathrm{~g}(\mathrm{c}$ and $\mathrm{f})$. The diffraction patterns were imaged at a rotation around the $x$-axis of $\phi=0^{\circ}$. The determined normalized lattice vectors $a_{1} / D(\mathrm{~d})$ and $a_{2 x} / D$ (e) are shown for all combinations of rubbing speeds $\left(v_{r}\right)$ and pressures $\left(p_{r}\right)$. The scale bar represents $5 \mu \mathrm{m}$. 
The determined values of $a_{1} / D$ and $a_{2 x} / D$, along with their standard deviations, for the lattices of the cube-like superballs are depicted in figure $4.3 \mathrm{~g}$ and $\mathrm{h}$ respectively for all combinations of rubbing speeds of $v_{r}=1,3$ and $5 \mathrm{~mm} / \mathrm{s}$ and pressures of $p_{r}=50,100,200$ and $400 \mathrm{~g}$. Similarly to the colloidal spheres it can be seen that the values for both $a_{1} / D$ and $a_{2 x} / D$ decrease upon increasing the rubbing pressure. Again, this is due to the particles being pushed together more strongly, resulting in a more densely packed lattice. The strongest changes in $a_{1} / D$ and $a_{2 x} / D$ are observed from an increase in the rubbing pressure from $p_{r}=50$ to $100 \mathrm{~g}$. Again, no clear trends can be seen in both $a_{1} / D$ and $a_{2 x} / D$ upon varying the rubbing speed $v_{r}$. For these cube-like superballs the 01 peak can still be identified a separate peak, instead of a continuous band as $h 1$, so that the angle $\alpha$ between the lattice vectors $\vec{a}_{1}$ and $\vec{a}_{2}$ can still be determined. Here, for each of the samples this angle ranges from $\alpha=117^{\circ}$ to $118^{\circ}$ which deviates significantly from $\alpha=120^{\circ}$ for an ideal hexagonal close-packed lattice of spherical particles as expected.

It can be concluded that for the preparation of superball monolayers from a rubbing pressure of at least $p_{r}=100 \mathrm{~g}$ is required is order to ensure the formation of well-defined lattices. The rubbing speed $v_{r}$, on the other hand, appears to less critical.

\subsubsection{Influence of superball shape and size}

We continue here with studying the influence of the shape and size of the superballs on the resulting monolayer lattice structures. In the following experiments we have used a rubbing speed of $v_{r}=3 \mathrm{~mm} / \mathrm{s}$ and pressure of $p_{r}=200 \mathrm{~g}$. These are the average values of the range that proved to be suitable for the preparation of colloidal superball monolayers with well-defined lattice structures as described in the previous subsection. First, in order to determine the influence of the shape of the superballs on the resulting monolayer lattice structures, we consider superballs with various shapes but with similar edge diameters. Representative SEM images of monolayers of colloidal superballs with various shapes prepared using the unidirectional rubbing method are depicted in figure 4.4. These superballs have an average shape parameter of $m=2,2.87 \pm 13 \%, 3.32 \pm 15 \%$ and $3.63 \pm 12 \%$ for those depicted in figure 4.4 a, b, c and d respectively. Their average edge diameters are $D=1.10 \pm 3.2 \%$, $0.96 \pm 4.5 \%, 1.13 \pm 5.3 \%$ and $1.01 \mu \mathrm{m} \pm 6.6 \%$. In figure 4.4 it can be seen that all four superball-shaped colloids had formed close-packed lattice structures when prepared at a rubbing speed of $v_{r}=3 \mathrm{~mm} / \mathrm{s}$ and pressure of $p_{r}=200 \mathrm{~g}$. Here, the colloidal silica spheres formed hexagonal close-packed lattices as can be seen in figure 4.4a. Similarly, the semi-cubic silica superballs also formed well-defined hexagonal-like close-packed lattices as can be seen in figure 4.4b. The more cubic-like superballs depicted in figure $4.4 \mathrm{c}$ and $\mathrm{d}$ did not purely 
assemble into hexagonal close-packed lattice structures, but instead assembled in the so-called sliding phase as already described in the previous chapter. In both figure $4.4 \mathrm{c}$ and d domains with a hexagonal-like lattice can be seen as well as domains with in a cubic lattice structure.
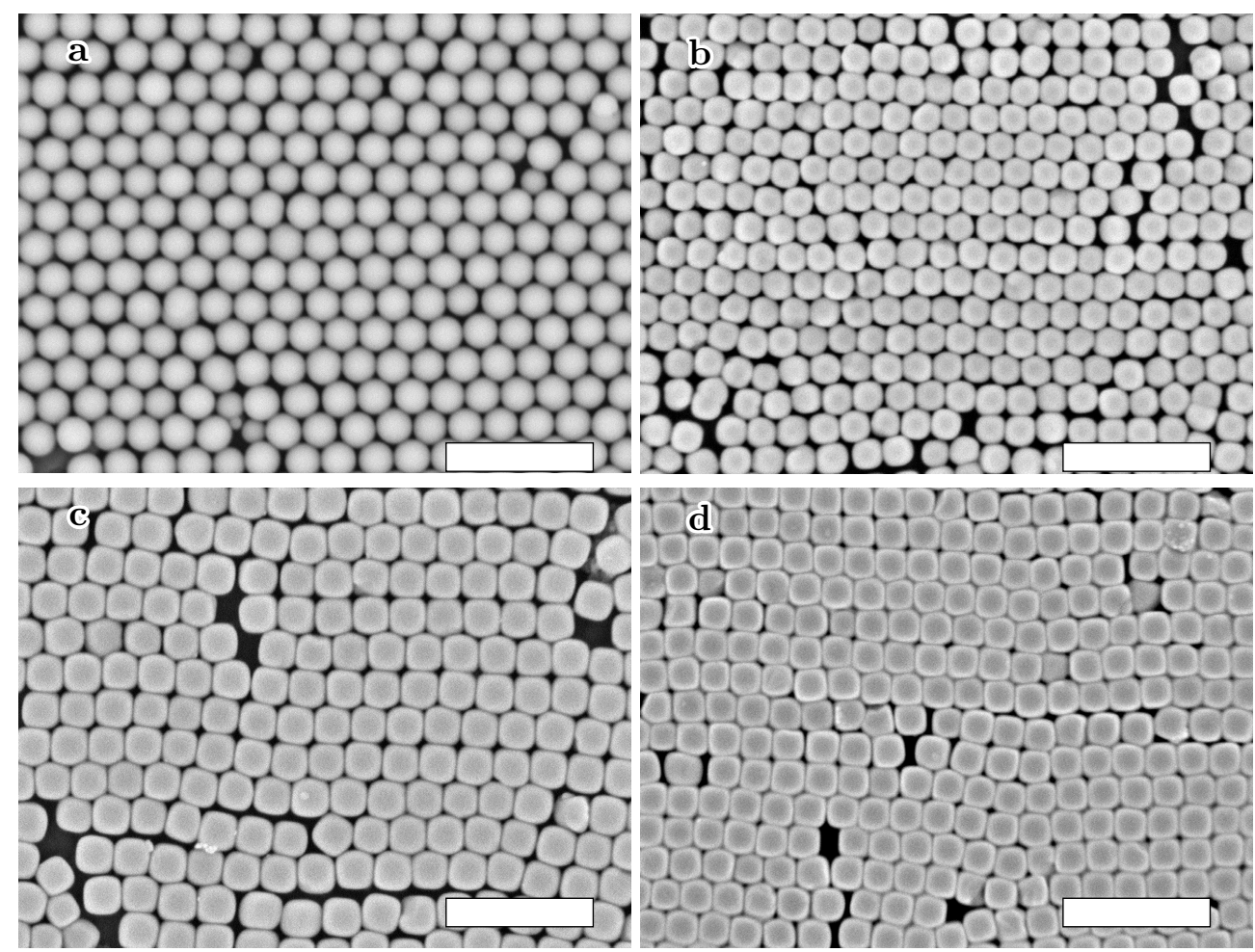

Figure 4.4: Representative SEM images of monolayers of colloidal superballs with various shapes prepared using the unidirectional rubbing method using a rubbing speed of $v_{r}=3 \mathrm{~mm} / \mathrm{s}$ and pressure of $v_{p}=200 \mathrm{~g}$. The superballs have an average shape parameter of $m=2$ (a), $2.87 \pm 13 \%$ (b), $3.32 \pm 16 \%$ (c) and $3.63 \pm 12 \%$ (d) and an average edge diameter of $D=1.10 \pm 3.2 \%$ (a), $0.96 \pm 4.5 \%$ (b), $1.13 \pm 5.3 \%$ (c) and $1.01 \mu \mathrm{m} 6.6 \%$ (d). These colloidal superball lattice structures were prepared by mechanical rubbing in the horizontal direction. The scale bars represent $5 \mu \mathrm{m}$.

In the images of the non-spherical superball lattices it can be seen that all particles are oriented with their flat faces towards the PDMS-coated surface. In fact, in all images not a single particle was observed to be attached to the PDMS-coated surface in any other orientation. This suggest that these superballs do not roll during their assembly into lattices but are instead pushed into their places. In all samples the assembled chains of particles are aligned in the rubbing direction as depicted in the horizontal direction of figure 4.4. In addition, it appears that within these chains the superballs strongly orient themselves with their faces in the direction of the applied rubbing. This may 
result from the particles having more contact area with the cylindrical rubbing tool as schematically shown in figure 2.4 on page 32 . Furthermore, it can be seen that the monolayers prepared from the more cubic-shaped superballs have a significant amount of defects where there is some empty space present between particles in the horizontal direction. These types of defects are not seen in the colloidal monolayers of the spherical superballs. This possibly results from the chains of the more cubic superballs not interlocking with each other as they are free to slide with respect to each other, while chains of spherical superballs are able to interlock.

To further study the influence of the superball shape and size on their assembled lattice structures we have used a larger variety of colloidal superballs with various shapes and sizes. Their average shape parameter $m$ and edge diameter $D$, along with their polydispersities, are listed in table 4.1. Note that not all combinations of shapes and sizes could be synthesized due to the limitations of the synthesis route as described in subsection 2.1.2 on page 24 .

\begin{tabular}{|c|c|}
\hline Average shape parameter $m$ & Average edge diameter $D(\mu \mathrm{m})$ \\
\hline 2 & $1.10 \pm 3.2 \%$ \\
2 & $1.36 \pm 4.0 \%$ \\
2 & $1.57 \pm 5.5 \%$ \\
$2.56 \pm 16 \%$ & $1.70 \pm 7.3 \%$ \\
$2.74 \pm 11 \%$ & $1.12 \pm 4.2 \%$ \\
$2.84 \pm 9 \%$ & $1.82 \pm 5.4 \%$ \\
$2.87 \pm 13 \%$ & $0.96 \pm 4.5 \%$ \\
$3.13 \pm 10 \%$ & $1.40 \pm 5.1 \%$ \\
$3.19 \pm 13 \%$ & $1.38 \pm 5.8 \%$ \\
$3.32 \pm 16 \%$ & $1.13 \pm 5.3 \%$ \\
$3.40 \pm 10 \%$ & $1.26 \pm 4.4 \%$ \\
$3.63 \pm 12 \%$ & $1.01 \pm 6.6 \%$ \\
\hline
\end{tabular}

Table 4.1: The average shape parameter $m$ and edge diameter $D$ of the colloidal superballs used to study the influence of the superball shape and size on the resulting lattice structures in colloidal superball monolayers prepared using the unidirectional rubbing method.

Alternatively, the average shape parameter $m$ and edge diameter $D$ of these colloidal superballs are depicted as a scatter plot in figure 4.5a to give a visual indication of the explored phase space. To quantitatively characterize the colloidal superball lattice structures in detail we again use SAXS. We first consider the ratio of $a_{1} / a_{2 x}$ which describes the ratio between the vertical distance between the chains that are aligned in the horizontal direction and the horizontal interparticle distance between adjacent particles in these chains as schematically shown previously in figure $3.6 \mathrm{f}$ on page 46 . This ratio is 
independent of the size of the superball particles. The determined ratio's of $a_{1} / a_{2 x}$ for the various superball shapes and sizes are depicted in figure $4.5 \mathrm{~b}$. The dashed line indicates $a_{1} / a_{2 x}$ for an ideal close-packed superball lattice as a function of the shape parameter $m$. For spheres arranged in a perfect hexagonal close-packed lattice structure this ratio is $a_{1} / a_{2 x}=0.866$ while for perfect cubes arranged in a cubic close-packed lattice this ratio is equal to 1 . The observed values of $a_{1} / a_{2 x}$ for the three spherical superballs agree very well with their ideal value of $a_{1} / a_{2 x}=0.866$. This again confirms that these type of superballs indeed form hexagonal lattices with a value of $\alpha$ that is close to its ideal value of $\alpha=120^{\circ}$, meaning that the superballs in the lattice are equally spaced apart in all directions.
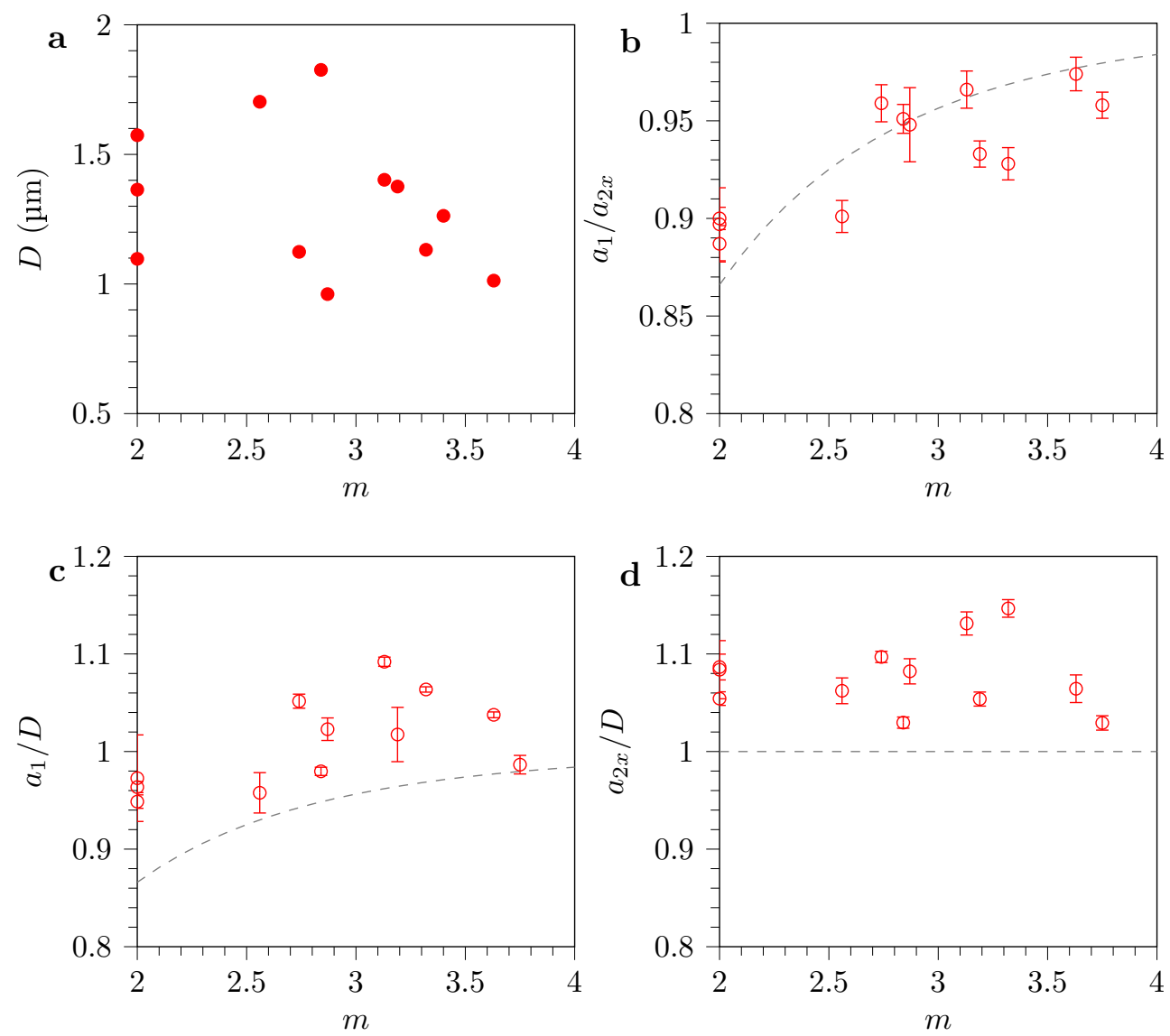

Figure 4.5: A visual representation of the average shape parameter $m$ and edge diameter $D$ of the colloidal superballs used to study the influence of the superball shape and size on the resulting lattice structures (a). The determined ratio of $a_{1} / a_{2 x}$ (b), $a_{1} / D$ (c) and $a_{2 x} / D$ (d) for the various superball lattices. Here, $a_{1}$ is the vertical distance between the chains of superballs, and $a_{2 x}$ is the interparticle distance between adjacent superballs in these chains. 
Likewise, the determined values of $a_{1} / a_{2 x}$ for most of the non-spherical superballs are close to the ideal value with some samples having a slightly lower value for $a_{1} / a_{2 x}$. Here, a lower values of $a_{1} / a_{2 x}$ compared to an ideal close-packed lattice implies that the monolayer structures are more strongly packed in the vertical direction compared to horizontal direction in which the rubbing force was applied. We, however, conclude here that there appears to be no significant deviation from the ideal value of $a_{1} / a_{2 x}$. Similarly to the spherical and cube-like superballs described in the previous subsection, we can again determine the values of $a_{1} / D$ and $a_{2 x} / D$ to gain a further understanding of the resulting lattice structures. The determined values of $a_{1} / D$ and $a_{2 x} / D$ for the lattice structures of superballs with the various shapes and sizes are depicted in figure $4.5 \mathrm{c}$ and $\mathrm{d}$ respectively. Again, $a_{1} / D$ describes the vertical distance between the chains normalized with the edge diameter $D$ of the superballs. The value of $a_{1} / D$ for an ideal close-packed hexagonal lattice of spheres also equals $a_{1} / D=0.866$ and increases upon increasing the shape parameter $m$ as represented by the dashed line depicted in figure $4.5 \mathrm{c}$. $a_{2 x} / D$, on the other hand, describes the interparticle distance between the superballs in the horizontal chains normalized with the edge diameter $D$ and, for an ideal close-packed lattice of superballs, is equal to 1 for all values of the shape parameter $m$ as represented by the dashed line in figure $4.5 \mathrm{~d}$. It can be seen that the determined values for both $a_{1} / D$ and $a_{2 x} / D$ are approximately between 5 and $10 \%$ higher than their ideal values represented by the dashed lines. The empty space between the particles is thus roughly between 5 and $10 \%$ of the diameter of the particles, corresponding to 50 to $100 \mathrm{~nm}$ for a lattice of superballs with an edge diameter of $D=1 \mu \mathrm{m}$. The presence of some empty space between the superballs in their lattice structures is not surprising as there are no significant attractive forces present between the colloidal superballs during their preparation with the unidirectional rubbing method. The colloidal particles are only shoved into place.

The influence of the superball size on the resulting lattice structures is less clear. For the spherical superballs, the differences in the values of $a_{1} / a_{2 x}$, $a_{1} / D$ and $a_{2 x} / D$ appear to be comparable to their standard deviations as seen in figure 4.5b, c and d. Furthermore, for the more cubic-like superballs with a shape parameter of around $m=3$, the differently sized superballs all have very similar values of $a_{1} / a_{2 x}, a_{1} / D$ and $a_{2 x} / D$. We can thus conclude that, unlike the superball shape, the size of the superballs has no significant influence on the resulting lattice structures.

While the length of the primitive basis vectors, $a_{1}$ and $a_{2 x}$, can be determined very accurately using SAXS, the exact degree of sliding present between the chains in the horizontal direction in the colloidal superball lattices cannot be determined with SAXS alone. In addition, the distribution in the positions of the neighbouring particles and the orientation of the non-spherical superballs are very difficult to determine using SAXS. Instead, we have employed 
SEM to study the local ordering of the colloidal superball lattice structures. In addition, SEM allows the orientation of the non-spherical superballs to be determined. We have performed particle tracking on the SEM images to determine the positions of the center of mass and the orientation of the superballs as described in subsection 4.2.3 on page 54. Examples of the processed SEM images with the tracked particles superimposed on top of the images are depicted in figure $4.6 \mathrm{a}, \mathrm{b}, \mathrm{c}$ and $\mathrm{d}$ for superballs with an average shape parameter of $m=2,2.87 \pm 13 \%, 3.32 \pm 16 \%$ and $3.63 \pm 12 \%$ respectively. These images are taken from the same colloidal superball monolayers as those depicted in figure 4.4 on page 61 . It can be seen that virtually all colloidal superballs are correctly identified as a particle by the script and that their positions and orientations appear to agree well with the outlines of the superballs seen in processed SEM images. Interestingly, we have found that the distribution in the orientation of the non-spherical superballs was independent of their shape and size. The standard deviation in the orientation of the non-spherical superballs was around $15^{\circ}$ for each of the samples. The similar standard deviation in the orientation of the superballs may also have resulted from the tracking procedure. As described in subsection 4.2.3, the tracking process of these images was relatively difficult as a significant amount of the particles appeared to have distorted shapes in their binarized images due to the high particle density. We therefore refrain from making any conclusions about the orientation of the superballs within the superball lattice structures.
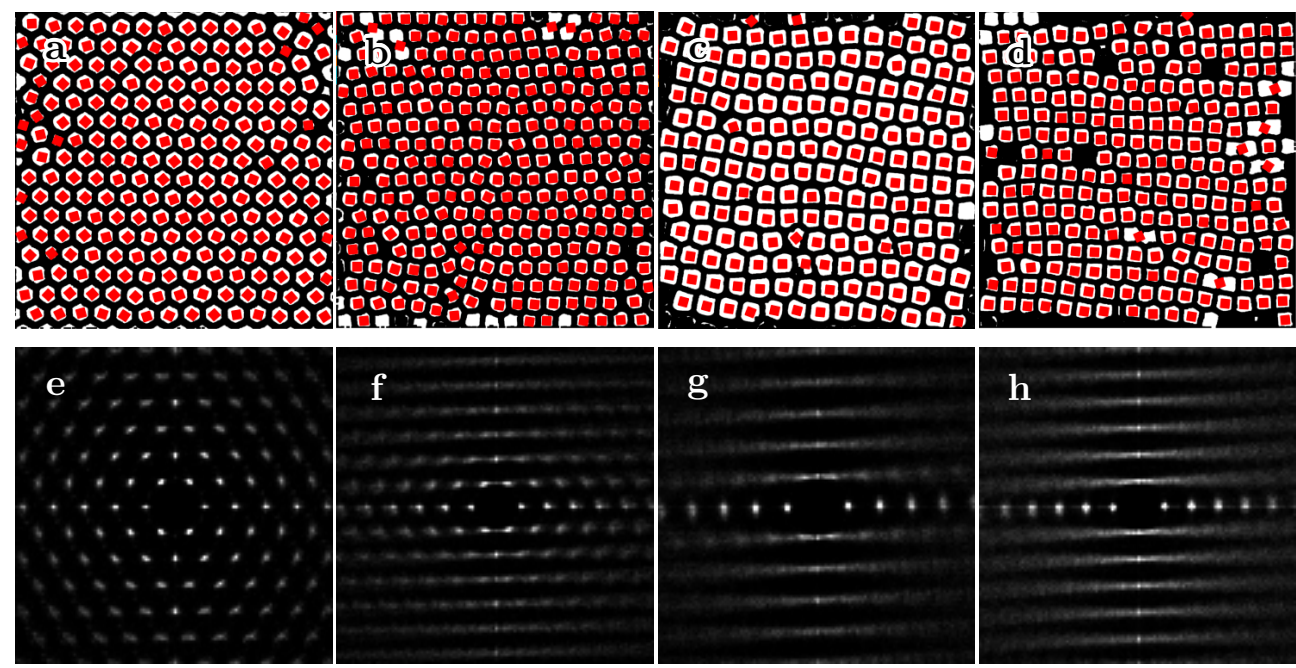

Figure 4.6: Examples of processed SEM images along with the tracked particles superimposed on top of the images (top row) and their determined two-dimensional pair distribution functions (bottom row). The superballs have an average shape parameter of $m=2(\mathrm{a}, \mathrm{e}), 2.87 \pm 13 \%(\mathrm{~b}, \mathrm{f}), 3.32 \pm 15 \%(\mathrm{c}, \mathrm{g})$ and $3.63 \pm 12 \%(\mathrm{~d}, \mathrm{~h})$ and an average edge diameter of $D=1.10 \pm 3.2 \%$ (a,e), $0.96 \pm 4.5 \%$ (b), $1.13 \pm 5.3 \%$ $(\mathrm{c}, \mathrm{g})$ and $1.01 \mathrm{\mu m} 6.6 \%(\mathrm{~d}, \mathrm{~h})$. 
The particles' the center of mass, however, could be determined relatively accurately as can already be seen by eye in the SEM images shown in figure $4.6 \mathrm{a}, \mathrm{b}, \mathrm{c}$ and $\mathrm{d}$. From the particles' center of mass positions we have calculated their two-dimensional pair distribution functions. The two-dimensional pair distribution functions of the colloidal superball lattices shown in figure $4.4 \mathrm{a}, \mathrm{b}, \mathrm{c}$ and $\mathrm{d}$ are depicted in figure 4.6e, $\mathrm{f}, \mathrm{g}$ and $\mathrm{h}$ respectively. These four two-dimensional pair distribution functions are normalized to account for the number of tracked superballs in each of the samples and depict the probability of a neighboring particle being at a certain position from the center of the image. Note here that the rubbing force was applied in the horizontal direction of the images. In the two-dimensional pair distribution function of the colloidal spheres, shown in figure 4.6e, it can be seen that the periodicity is well-defined, both in the vertical and horizontal direction. Upon increasing the shape parameter $m$ of the superballs, the periodicity in the horizontal direction remains, as evidenced by the sharp peaks in the the horizontal direction. In the vertical direction, however, it can be seen that the peaks broaden upon increasing the shape parameter $m$ of the superballs and eventually begin to form continuous bands as can be seen in figure 4.6f, g and h. In the two-dimensional pair distribution functions of the more cube-like superballs, where these chains are able to slide more freely with respect to each other, the continuous band appears to be very thin and intense directly above the particle that is being considered. This may result from particles at this position having less positional freedom in the vertical direction when being directly above or below the face of a neighboring particle in the vertical direction.

The degree of which the chains in the horizontal direction are able to slide with respect to each other can be studied by considering the cross sections along each row of the peaks in the horizontal direction of the two-dimensional pair distribution functions. The first horizontal cross sections of the discussed two-dimensional pair distribution functions are depicted in figure 4.7a. These plots describe the relative probability of a certain shift present between the chains in the horizontal direction. The $x$-axis of this plot, $r_{x} / s_{2 x}$, is normalized with $s_{2 x}$, which is the horizontal interparticle distance and analogous to $a_{2 x}$, but determined using the two-dimensional pair distribution function. Two sharp peaks are observed for the lattice structures of the spherical superballs. Upon increasing the shape parameter $m$ these peaks can be seen to broaden and eventually form a continuous profile. Interestingly, for the more cubicshaped superballs peaks begin to occur at a relative shift of $r_{x} / s_{2 x}=0$, which hints to a small preference for the cubic lattice. We can calculate the standard deviation of these profiles to quantify the exact degree at which the chains are able to slide with respect to each other. Here, have we determined the standard deviation $\sigma_{p}$ in the profiles from $r_{x} / s_{2 x}=0$ to 1 . For an ideal hexagonal or cubic lattice structure the peaks in the two-dimensional pair 
distribution function are infinitely sharp and the resulting standard deviation is then $\sigma_{p}=0$. For an uniform distribution, corresponding to chains that are able to slide freely with respect to each other with no preferential position, the standard deviation is $\sigma_{p}=1 / \sqrt{12} \approx 0.29$. The standard deviation can therefore be used to quantify the degree of sliding. The determined values of $\sigma_{p}$ for the lattice structures of superballs with various shapes and sizes as listed in table 4.1 on page 62 are depicted in figure $4.7 \mathrm{~b}$. For the lattice structures of the three spherical colloidal superballs we find that $\sigma_{p}$ is around 0.1 , which shows that the colloidal spheres have a small amount of positional freedom in the horizontal direction. It can clearly be seen that $\sigma_{p}$ increases upon increasing the shape parameter $m$ of the superballs. For the most cubic superballs, such as those with a shape parameter of $m=3.63$, we indeed find that its value of $\sigma_{p}$ is close to that of an uniform distribution and that its chains are thus free to slide randomly with respect to each other. This is plausible as we have shown earlier that the distance between the chains of superballs formed in the horizontal direction is approximately 5 to $10 \%$ larger than their edge diameter, which thus provides enough space for the chains to slide with respect to each other. Finally, it can be seen that $\sigma_{p}$ appears to increase approximately linear with the shape parameter $m$. We can thus conclude that the transition from the hexagonal close-packed lattice formed for colloidal spheres to the sliding phase formed for cube-like superballs with a shape parameter of around $m=3.5$ is thus smooth and continuous.
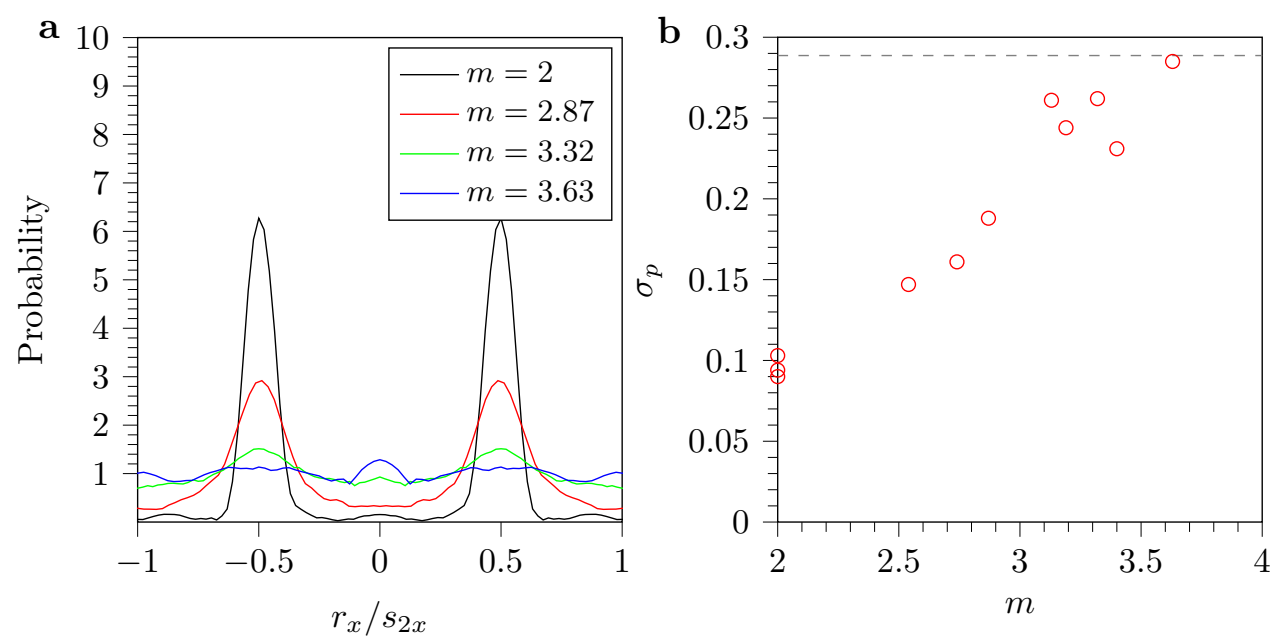

Figure 4.7: Normalized cross sections of the first row of peaks along the horizontal direction of the two-dimensional pair distribution functions of the lattice structures of colloidal superballs with various shape parameters $m$ (a) and the calculated standard deviation $\sigma_{p}$ in their profiles from $r_{x} / s_{2 x}=0$ to 1 . The dashed line in (b) corresponds to a standard deviation of $\sigma_{p}=1 / \sqrt{12} \approx 0.29$ for an uniform distribution. 


\subsection{Conclusion}

Monolayers of colloidal superballs with various shapes and sizes were prepared using the unidirectional rubbing method in order to study the influence of their shape and size on the resulting lattice structures. In addition, these monolayers were prepared at various rubbing speeds and pressures to study the effect of these parameters on the superball lattice structures. We have studied the resulting lattice structures with both SAXS and SEM which are two complementary techniques. It was observed that well-defined hexagonal close-packed lattices resulted for colloidal spheres using all combinations of rubbing speeds between $v_{r}=1$ and $5 \mathrm{~mm} / \mathrm{s}$ and pressures between $p_{r}=50$ and $400 \mathrm{~g}$. From the SAXS measurements it was observed that the lattice vectors $a_{1}$ and $a_{2 x}$ decreased slightly upon increasing the rubbing pressure $p_{r}$, resulting in the formation of slightly denser lattices at a higher rubbing pressure. For superballs with a cube-like shape and an average shape parameter of $m=$ 3.40 we found that a relatively low rubbing pressure of $50 \mathrm{~g}$ yields colloidal monolayers with a poorly defined periodicity as apparent from both the SEM images and the SAXS diffraction patterns. In the SEM images it was observed that upon increasing the rubbing pressure to at least $p_{r}=100 \mathrm{~g}$ the lattice structures become better defined and in the corresponding diffraction patterns it was seen that the Bragg peaks became much sharper. Here, the values for the lattice vectors $a_{1}$ and $a_{2 x}$ also decreased upon increasing the rubbing pressure to at least $p_{r}=100 \mathrm{~g}$. It can thus be concluded that more cubic superballs require more force to be assembled into colloidal monolayers with a well-defined periodicity. In our experiments of both the spherical and cubelike superballs, the rubbing speed $v_{r}$ had no clear influence on the lattice structures.

The influence of the shape and size of the superballs on the lattice structures was studied in more detail using superballs with various shapes and sizes prepared at a constant rubbing speed of $3 \mathrm{~mm} / \mathrm{s}$ and pressure of $p_{r}=200 \mathrm{~g}$. It was observed that upon increasing the shape parameter $m$ of the superballs the values of $a_{1} / a_{2 x}$ increased as expected. Here, $a_{1}$ describes the distance between the chains formed along the horizontal direction and $a_{2 x}$ is the interparticle distance between the superballs in these chains. For all shapes and sizes of superballs the values of $a_{1} / D$ and $a_{2 x} / D$ were approximately 5 to $10 \%$ higher than expected based on an ideal close-packed lattice of superballs. Here, the amount of empty space between the particles possibly results from the lack of any attraction present between the particles during their assembly using the unidirectional rubbing method. The only force comes from the mechanical rubbing motion. From both the SAXS diffraction patterns and the two-dimensional pair distribution functions determined from the SEM images it was observed that the periodicity in the lattice structures of the colloidal 
superballs in the horizontal direction remained but that the periodicity in the vertical direction vanished upon increasing the shape parameter $m$. A continuous transition was observed from the hexagonal lattice formed for spherical superballs to the so-called sliding phase for the more cube-like superballs. At a shape parameter of around $m=3.5$ the chains of superballs formed in the horizontal direction were able to slide completely free with respect to each other as apparent from the cross sections of the two-dimensional pair distribution functions. In future research it might be interesting to image the formation of these superball lattice structures in situ using, for example, optical microscopy to gain an insight into the mechanism of the unidirectional rubbing method.

\subsection{Acknowledgements}

Janne-Mieke Meijer is thanked for the fruitful discussions, her help with the analysis of the data and for checking the writing. Dominique Thies-Weesie is thanked for her help with the preparation of the spherical silica colloids. Nicholas Orr is thanked for his help at the beamline of the ESRF. Michela Brunelli and Daniel Hermida Merino are thanked for providing technical support at the beamline. The Nederlandse Organisatie voor Wetenschappelijk Onderzoek (NWO) is acknowledged for providing the SAXS beamtime.

\section{References}

[1] A. van Blaaderen, "Colloids get complex," Nature, vol. 439, no. 7076, pp. 545546, 2006.

[2] S. Sacanna, D. J. Pine, and G. R. Yi, "Engineering shape: The novel geometries of colloidal self-assembly," Soft Matter, vol. 9, no. 34, pp. 8096-8106, 2013.

[3] M. A. Boles, M. Engel, and D. V. Talapin, "Self-assembly of colloidal nanocrystals: From intricate structures to functional materials," Chemical Reviews, vol. 116, no. 18, pp. 11220-11 289, 2016.

[4] S. Jiang, A. Van Dyk, A. Maurice, J. Bohling, D. Fasano, and S. Brownell, "Design colloidal particle morphology and self-assembly for coating applications," Chemical Society Reviews, vol. 46, no. 12, pp. 3792-3807, 2017.

[5] J. M. Meijer, D. V. Byelov, L. Rossi, A. Snigirev, I. Snigireva, A. P. Philipse, and A. V. Petukhov, "Self-assembly of colloidal hematite cubes: A microradian Xray diffraction exploration of sedimentary crystals," Soft Matter, vol. 9, no. 45, pp. 10729-10738, 2013.

[6] J. M. Meijer, A. Pal, S. Ouhajji, H. N. Lekkerkerker, A. P. Philipse, and A. V. Petukhov, "Observation of solid-solid transitions in 3D crystals of colloidal superballs," Nature Communications, vol. 8, pp. 1-8, 2017. 
[7] L. Rossi, S. Sacanna, W. T. Irvine, P. M. Chaikin, D. J. Pine, and A. P. Philipse, "Cubic crystals from cubic colloids," Soft Matter, vol. 7, no. 9, pp. 4139-4142, 2011.

[8] L. Rossi, V. Soni, D. J. Ashton, D. J. Pine, A. P. Philipse, P. M. Chaikin, M. Dijkstra, S. Sacanna, and W. T. Irvine, "Shape-sensitive crystallization in colloidal superball fluids," Proceedings of the National Academy of Sciences of the United States of America, vol. 112, no. 17, pp. 5286-5290, 2015.

[9] J. M. Meijer, F. Hagemans, L. Rossi, D. V. Byelov, S. I. Castillo, A. Snigirev, I. Snigireva, A. P. Philipse, and A. V. Petukhov, "Self-assembly of colloidal cubes via vertical deposition," Langmuir, vol. 28, no. 20, pp. 7631-7638, 2012.

[10] J. M. Meijer, V. Meester, F. Hagemans, H. N. Lekkerkerker, A. P. Philipse, and A. V. Petukhov, "Convectively assembled monolayers of colloidal cubes: Evidence of optimal packings," Langmuir, vol. 35, no. 14, pp. 4946-4955, 2019.

[11] Y. Jiao, F. H. Stillinger, and S. Torquato, "Optimal packings of superdisks and the role of symmetry," Physical Review Letters, vol. 100, no. 24, pp. 2-5, 2008.

[12] C. Park, T. Lee, Y. Xia, T. J. Shin, J. Myoung, and U. Jeong, "Quick, large-area assembly of a single-crystal monolayer of spherical particles by unidirectional rubbing," Advanced Materials, vol. 26, no. 27, pp. 4633-4638, 2014.

[13] C. Park, K. Koh, and U. Jeong, "Structural Color Painting by Rubbing Particle Powder," Scientific Reports, vol. 5, pp. 1-5, 2015. 


\section{Chapter 5}

\section{Active Colloidal Circlers and Spinners}

\section{Abstract}

The behaviour of active systems is determined by the properties of the individual particles. The ability to program the behaviour of the individual particles should therefore allow the formation of complex self-assembled structures. In this chapter, we show that active colloids can be made to self-propel into tunable, circular trajectories under the influence of alternating electric fields by sputter coating their colloidal monolayers with a thin layer of gold under various glancing angles. We demonstrate how the velocity and angular velocity of these effectively chiral particles can be tuned in situ by varying the magnitude and frequency of the applied alternating electric field. Furthermore, we show that the remaining velocity can be further decreased and virtually eliminated by sputter coating both hemispheres of the colloidal particle with an asymmetrical gold patch instead. These active colloidal spinners almost purely display a rotational motion. The development of such programmable particles opens up the possibilities for steerable and programmable colloidal systems and self-assembled structures. 


\subsection{Introduction}

Active colloids convert the energy available in their environment, whether chemical or physical, into motion. Their behaviour is determined by their design [1-3]. While the vast majority of work in the field of active matter focusses on active colloids with straight trajectories, active colloids with nonlinear active motion have also been described in the literature. For example, self-propelled L-shaped particles were shown to be swimming in circular trajectories due to the asymmetrical drag on the particles [4]. In addition, it has been shown more recently that by sputter coating colloidal monolayers under a glancing angle, asymmetrically-shaped metal patches can be deposited on the colloidal spheres $[5,6]$. When sputter coated with platinum, the resulting colloidal particles were observed to be self-propelled into circular trajectories in solutions of hydrogen peroxide.

Active colloids are able to form a rich variety of phases, depending on their number density and their individual properties, as already discussed in the general introduction. Briefly, the most well-known and frequently studied phase is the so-called motility-induced phase separation (MIPS) which occurs at a sufficiently high number density and activity [7-10]. In MIPS, the active colloids cluster due to the occurrence of collisions between the particles. The introduction of attractive and repulsive interactions has been shown to result in a variety of self-assembled phases such as, for example, swarming and the formation of linear chains [11]. Furthermore, active colloids have been shown to be able to assist self-assembly processes $[12,13]$. The development of active colloids with more complex motion on the individual level may result in different and possibly more complex phase behaviour on a collective level and the discovery of novel phases. Such active colloidal systems may in the future be used in various applications such as, for example, the development of sensors for chemical or physical stimuli, drug delivery and the development of smart materials $[3,14,15]$.

The use of hydrogen peroxide as a fuel source for active colloids, however, limits their applicability as the resulting particles are difficult to tune in situ. Moreover, fuel depletion and the formation of oxygen bubbles limits the number density of the particles at which these model systems can be studied. The use of a physical fuel source solves both of these difficulties as external stimuli are generally more easily tuned and leave no waste products [3]. For example, alternating electric fields can be used to power active colloids with a metallic patch [11]. These colloids become self-propelled due to induced-charge electrophoresis (ICEP) as a result of uneven ionic flows around both hemispheres of the colloidal particles [16]. An advantage of this model system is that the magnitude and frequency of the alternating electric field can be controlled in situ very easily, which determines their behaviour on both an individual and collective level $[11,16]$. 
In this chapter we demonstrate that alternating electric fields can also be employed to power active colloids with circling active motion. We prepare these so-called active colloidal circlers by sputter coating monolayers of large colloidal poly(methyl methacrylate) spheres with a low polydispersity under a glancing angle with a thin layer of gold in order to introduce asymmetrical patches. The particles' relatively large diameter allows both the Brownian translational and rotational motion to be minimized, which increases the visibility of their anisotropic active motion. The colloids were assembled into colloidal monolayers using the unidirectional rubbing method. As seen in the previous chapters, the unidirectional rubbing method allows colloidal monolayers with a well-defined periodicity to be produced with the crystal grains aligned in the direction of rubbing. The use of the unidirectional rubbing method, in combination with the relatively low polydispersity of the colloids, should minimize the variation in the shape of the applied metallic patch. The minimization in the shape of this patch should, in turn, minimize the spread in the velocity and angular velocity of the resulting active colloidal circlers. We study the effect of the glancing angle on the resulting active behaviour as well as the influence of both the magnitude and frequency of the applied alternating electric field. Finally, we show that by applying a second metallic patch on the opposite side of the colloidal particle, the remaining translational motion can be virtually eliminated. These so-called active colloidal spinners only display rotational motion under the influence of an alternating electric field.

\subsection{Experimental methods}

\subsubsection{Preparation of the active colloidal circlers}

Colloidal poly(methyl methacrylate) spheres were prepared using dispersion polymerization and analysed as described in section 2.2. The average diameter of these colloids was $d=4.0 \mu \mathrm{m}$ and the polydispersity in the diameter was $\sigma_{d}=2.5 \%$. Small amounts of the resulting colloidal dispersion were dried under a nitrogen flow and used for the assembly into monolayers using the unidirectional rubbing method as described in section 2.4. The colloids were assembled into monolayers on polydimethylsiloxane- (PDMS) coated glass slides $(\# 0,60 \mathrm{~mm} \times 24 \mathrm{~mm} \times 0.085-0.12 \mathrm{~mm}$ ) which were prepared as described in section 2.3. For all samples the device was set to run 10 rubbing cycles at a rubbing pressure of $p_{r}=200 \mathrm{~g}$ and a rubbing speed of $v_{r}=3 \mathrm{~mm} / \mathrm{s}$. The resulting colloidal monolayers were then coated with a $15 \mathrm{~nm}$ thick layer of gold using a Leica EM ACE600 sputter coater under various glancing angles $\theta$. Here, the glancing angle of the sputter coating was along the direction of the mechanical rubbing with an angle of $\theta=0^{\circ}$ being defined as coated normally to the monolayer plane. The maximum achievable 
glancing angle was $\theta=40^{\circ}$ using this sputter coater. The now partially with gold coated colloids were then dispersed into an aqueous solution containing $0.1 \mathrm{~g} /$ L Pluronic F127 (Sigma) by ultrasonicating a piece of the PDMS-coated glass slide containing the colloidal monolayer into the Pluronic F127 solution for 1 minute. The synthesis process is schematically illustrated in figure 5.1.

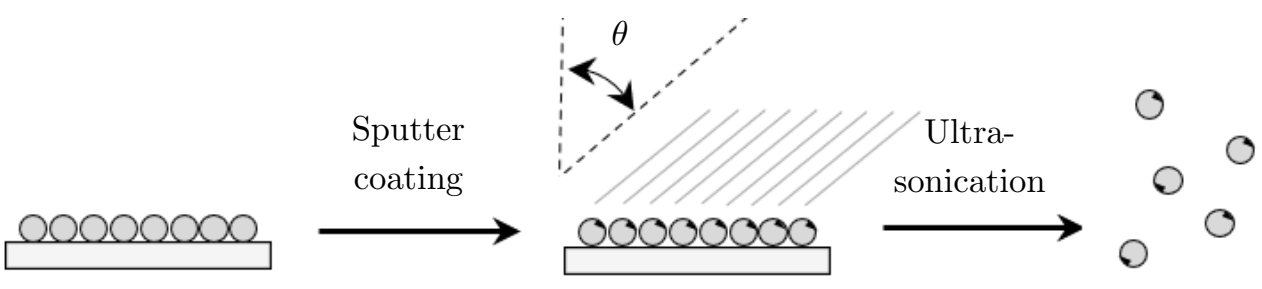

Figure 5.1: A schematic illustration of the synthesis process of the active colloidal circlers. The colloidal spheres are first assembled into colloidal monolayers on a PDMS-coated substrate using the unidirectional rubbing method. The colloidal monolayer is then sputter coated with $15 \mathrm{~nm}$ of gold under various glancing angles of $0^{\circ}<\theta<40^{\circ}$. Finally, the PDMS-coated glass slide is ultrasonicated in order to release the resulting active colloidal circlers from the substrate and to disperse them into solution.

\subsubsection{Preparation of the active colloidal spinners}

To further reduce the remaining translational motion of these type of active colloids, we introduce an adaptation in their synthesis process. These so-called active colloidal spinners are prepared by also sputter coating an asymmetrical patch on the opposite side of the particle. A similar method has previously been used to prepare such colloids, where they were used to study a static selfassembly process [17]. The colloidal spheres were first assembled into monolayers on PDMS coated glass slides (\#0, $60 \mathrm{~mm}$ x $24 \mathrm{~mm} \times 0.085-0.12 \mathrm{~mm}$ ), prepared by mixing the silicone elastomer base and the silicone elastomer curing agent from the Sylgard 184 silicon elastomer kit in a ratio of 5:1 by weight. The colloidal monolayers were then sputter coated with $10 \mathrm{~nm}$ of platinum under a glancing angle of $\theta=60^{\circ}$ using a Cressington 208HR sputter coater. The now coated colloidal monolayers were then inverted and pressed onto a second PDMS-coated glass slide that had a much more sticky surface. By mixing the silicon elastomer base and the silicone elastomer curing agent in a ratio of 40:1 by weight, a much more sticky PDMS coating was obtained. Care was taken not to slide the PDMS-coated glass slides with respect to each other during the transfer of the particles, as this might disturb the orientation of the colloids in the monolayers. After transferring the colloidal monolayer to the second PDMS-coated glass slide, the colloidal monolayers were sputter coated for a second time with $10 \mathrm{~nm}$ of platinum under a glancing angle of $\theta=60^{\circ}$. Finally, the resulting colloids were dispersed in an aqueous solution containing $0.1 \mathrm{~g} / \mathrm{L}$ 
Pluronic F127 (Sigma) by ultrasonicating a piece of the PDMS-coated glass slide containing the colloidal monolayer into the Pluronic F127 solution for 1 minute. The synthesis process is schematically illustrated in figure 5.2.

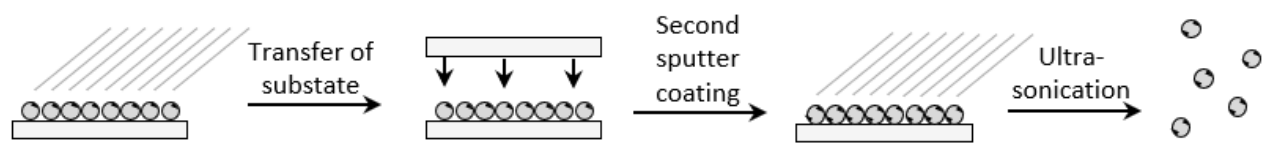

Figure 5.2: A schematic illustration of the synthesis process of the active colloidal spinners. The colloidal spheres are first assembled into colloidal monolayers on a PDMS-coated substrate using the unidirectional rubbing method. The colloidal monolayer is then sputter coated with $10 \mathrm{~nm}$ of platinum under a glancing angle of $\theta=60^{\circ}$. The colloidal monolayer was then pressed onto another and more stickier PDMS-coated glass slide. The colloidal particles transfer to the more sticky surface, and the colloidal monolayers are sputter coated a second time with $10 \mathrm{~nm}$ of platinum under the same glancing angle. The PDMS-coated glass slide is finally ultrasonicated in order to release the active colloidal spinners from the substrate and to disperse them into solution.

\subsubsection{Production and loading of the electric field cell}

Dispersions containing the active colloids were diluted to a suitable number density of active colloids and to a concentration of $0.1 \mathrm{~g} / \mathrm{L}$ Pluronic F127. Pluronic F127 is a hydrophilic, non-ionic surfactant added to reduce the particles' tendency to stick to ITO-coated surfaces. A small volume of the resulting dispersion was then used to fill the custom-made cells consisting of two ITO-coated glass slides spaced $100 \mu \mathrm{m}$ apart with the conductive ITO surfaces on the inside of the cell and in direct contact with the dispersion as presented in section 2.5. An alternating electric field was applied between the ITO electrodes using a Yokogawa FG120 function generator coupled to an Ohmtronics 7602M wideband amplifier. A Yokogawa DLM2022 digital oscilloscope was used to determine the amplitude of the applied alternating electric field.

\subsubsection{Analysis of the particle trajectories}

The active motion of these colloids were imaged using a Nikon Eclipse Ti-U optical microscope equipped with Nikon 40x, 20x and 10x objectives, depending on the activity of the active colloids. A frame rate of $10 \mathrm{fps}$ was used for all samples studied. A custom-written Matlab script was used in order to track the positions of the particles in time. For each particle, the mean squared displacement (MSD) of the particles' trajectory as a function of the time interval 
$\Delta t$ was calculated as follows:

$$
\Delta r^{2}(\Delta t)=\left\langle(\vec{x}(t+\Delta t)-\vec{x}(t))^{2}\right\rangle_{t}=\frac{1}{N} \sum_{n=1}^{N}\left(\vec{x}\left(t_{n}+\Delta t\right)-\vec{x}\left(t_{n}\right)\right)^{2},
$$

where $N$ is the number of steps to average over and $\vec{x}(t+\Delta t)$ and $\vec{x}(t)$ are the positions of the particle in two dimensions at $t_{n}+\Delta t$ and $t_{n}$ respectively, where $t_{n}$ is the variable start of the time interval. Trajectories where the particles had become too close to any other particle at any time were removed to prevent the influence of colloidal interactions. The experimental data was then fitted to the temporal evolution of the mean squared displacement as follows $[6]$ :

$$
\begin{aligned}
\Delta r^{2}(\Delta t)=4 D_{0} t+\frac{2 v^{2} D_{r} t}{D_{r}^{2}+\omega^{2}} & +\frac{2 v^{2}\left(\omega^{2}-D_{r}^{2}\right)}{\left(D_{r}^{2}+\omega^{2}\right)^{2}} \\
& +\frac{2 v^{2} \mathrm{e}^{-D_{r} t}}{\left(D_{r}^{2}+\omega^{2}\right)^{2}}\left[\left(D_{r}^{2}-\omega^{2}\right) \cos \omega t-\omega D_{r} \sin \omega t\right]
\end{aligned}
$$

where $D_{0}$ is the Brownian translational diffusion coefficient, $D_{r}$ is the Brownian rotational diffusion coefficient, $v$ is the velocity and $\omega$ is the angular velocity of the active motion.

\subsection{Results and discussion}

\subsubsection{Influence of the glancing angle on the active behaviour}

We begin by studying the influence of the glancing angle $\theta$ at which the colloidal monolayers are sputter coated on the behaviour of the resulting active colloids. By sputter coating under a glancing angle, an asymmetry in the shape of the resulting metal patch can be obtained. The active colloids described in this subsection were studied under the influence of an alternating electric field with a magnitude of $E_{p t p}=120 \mathrm{~V} / \mathrm{mm}$ and a frequency of $3 \mathrm{kHz}$. Trajectories of representative examples of active colloids prepared by sputter coating under glancing angles of $\theta=0,20$ and $40^{\circ}$ are depicted in figure 5.3a. It can be seen here that the active colloid sputter coated under a glancing angle of $\theta=0^{\circ}$ became self-propelled into a straight trajectory as expected due to the particles symmetrical coating. The particles sputter coated under glancing angles of $\theta>0^{\circ}$, on the other hand, were observed propel themselves in circular trajectories as expected resulting from their asymmetrically-shaped gold patch. The radii in which these active colloids circle decreases upon increasing the glancing angle at which these colloids were prepared. The mean squared displacements of these three particles, as calculated using equation 5.1, are 
depicted in figure 5.3b. It can be seen that the mean squared displacement of the particles coated under a glancing angle of $\theta=0^{\circ}$ increases quadratically on short time scales and linearly on longer timescales. On a short time scale, the particle self-propels into a straight direction and on this time the particle is ballistic. On longer time scales, however, the particle loses its directional motion due to rotational diffusion. It can be seen that the active colloids that were coated under a glancing angle $\theta>0^{\circ}$ begin to display oscillations in their mean squared displacements. Here, on average, the mean squared displacement also increases in time due to the presence of rotational diffusion. These random rotational motions cause a drift in the circular motion of the particles, which can also be seen in their trajectories. The rotational diffusion of the particles studied here are relatively slow, however, due to their relatively large size. The velocity and angular velocity of the active colloids were determined by fitting the temporal evolution of the mean squared displacement, presented in equation 5.2, on the calculated MSD. In figure 5.3b it can be seen that the fit agrees very well with experimental data. From the fit we determined the velocities to be $v=34.2,31.5$ and $29.7 \mu \mathrm{m} / \mathrm{s}$ for the particles coated under a glancing angle of $\theta=0,20$ and $40^{\circ}$ respectively. Their angular velocities were determined to be $\omega=0.02,0.23$ and $0.66 \mathrm{rad} / \mathrm{s}$ respectively.

a

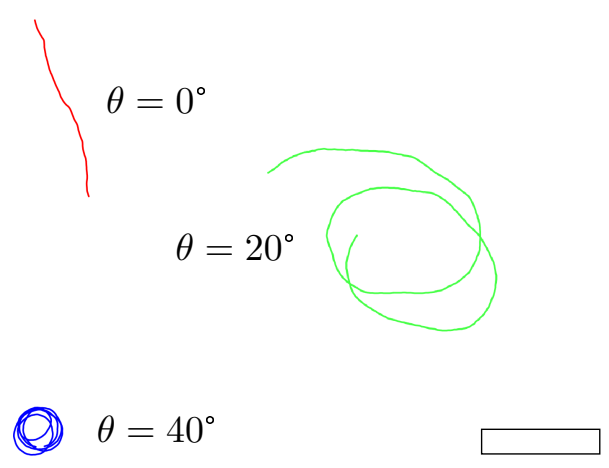

b

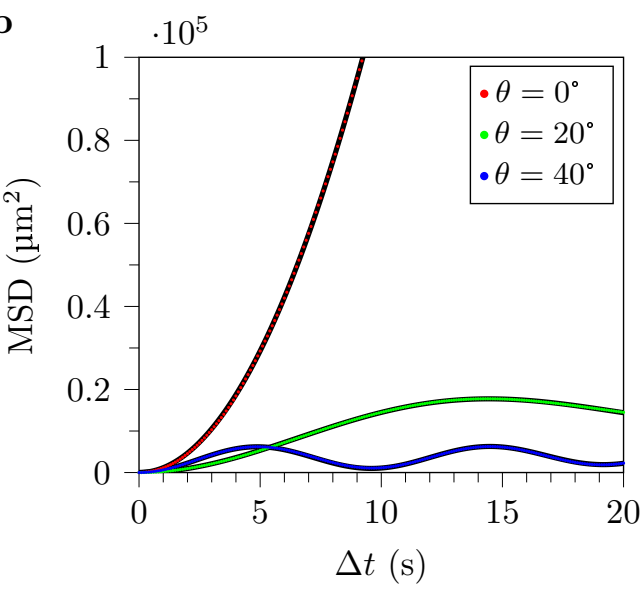

Figure 5.3: Examples of trajectories of representative active colloidal circlers, prepared by sputter coating colloidal monolayers under glancing angles of $\theta=0,20$ and $40^{\circ}$, under the influence of an alternating electric field with a magnitude of $E_{p t p}=120 \mathrm{~V} / \mathrm{mm}$ and frequency of $f=3 \mathrm{kHz}(\mathrm{a})$. The mean squared displacements of these three particles (coloured marks) along with their fits (black lines) are depicted in (b). The scale bar in (a) represents $100 \mu \mathrm{m}$.

The average velocity and angular velocity of the particles prepared by sputter coating their monolayers with gold under the various glancing angles $\theta$ are depicted in figure $5.4 \mathrm{a}$ and b respectively. Here, at least 20 particles 
were used for each of the glancing angles in order to accurately determine the average velocity and angular velocity of the particles, along with their standard deviations. It can be seen that the average velocity of the active colloids decreases slightly upon increasing the glancing angle. For each of the samples, the standard deviation in the velocity was between 15 and $20 \%$. The average angular velocity, on the other hand, increases from $\omega=0.11 \pm$ $100 \%$ to $0.42 \pm 50 \%$ to $1.08 \pm 35 \% \mathrm{rad} / \mathrm{s}$ as can be seen in figure $5.4 \mathrm{~b}$. The average radii of the circular trajectories can be calculated as $r=v / \omega$, with which we find that the average radii are $r=342,73$ and $25 \mu \mathrm{m}$ for the active colloids prepared under a glancing angle of $\theta=0,20$ and $40^{\circ}$ respectively. The non-zero value calculated for the angular velocity, and the finite radii as result, for the active colloids sputter coated symmetrically at $\theta=0^{\circ}$ likely result from the presence of small defects in the coating, which introduces small angular velocities. The standard deviations observed in the velocity and angular velocity are similar to those found by Archer et al. in their hydrogen peroxide fueled active colloidal circlers [6]. Considering that ICEP is known to be sensitive on the shape and thickness of the coating, the obtained active colloidal circlers seem promising for, for example, self-assembly studies where identical behaviour of the individual particles is generally desired.
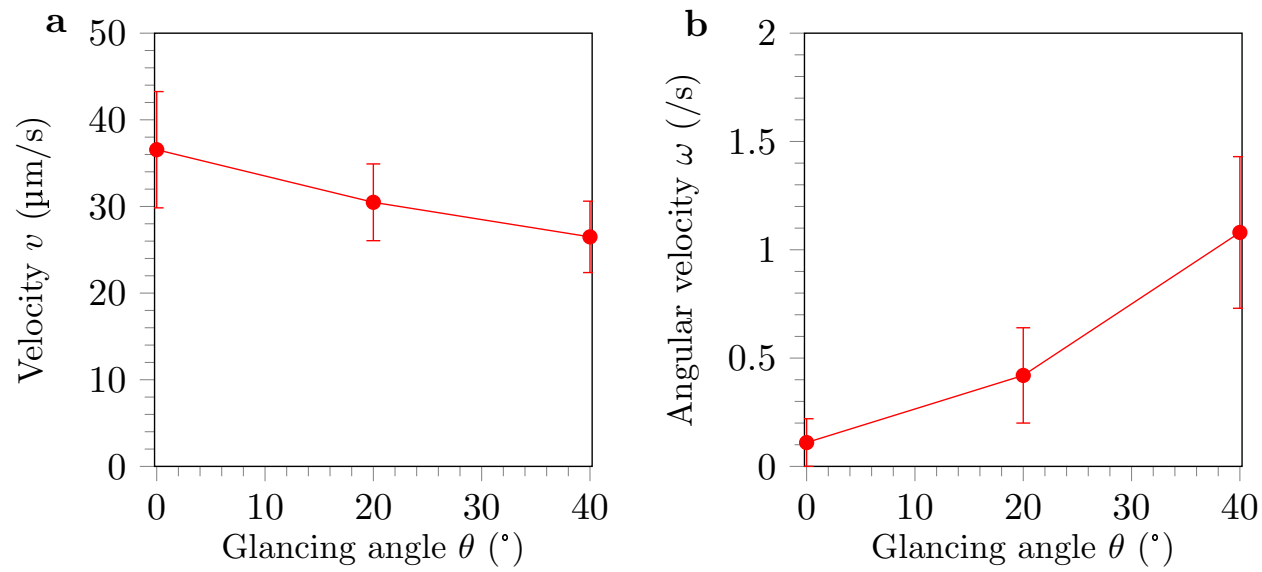

Figure 5.4: The average velocity (a) and angular velocity (b) of active colloidal circlers prepared by sputter coating their monolayers with gold under glancing angles of $\theta=0,20$ and $40^{\circ}$. All samples were studied under the influence of an alternating electric field of magnitude $E_{p t p}=120 \mathrm{~V} / \mathrm{mm}$ and frequency $f=3 \mathrm{kHz}$.

In the trajectories of the active colloidal circlers that were prepared by sputter coating their monolayers under a glancing angle of $\theta>0^{\circ}$, such as for example depicted in figure 5.3a, it can be seen that the direction in which the particles circle does not change from clockwise to anticlockwise or vice versa. In fact, we have not seen any particles in any of our experiments that were able to switch the direction of their circular motion. Upon removing and 
reapplying the alternating electric field, however, the particles were observed to be able to switch their direction of circling. This results from the gold patch of the particle being aligned in the direction of the alternating electric field. This pinning prevents the rotational motion around the $x$ - and $y$-axes and only allows rotation around the $z$-axis. This feature makes these active colloidal circlers effectively chiral in two dimensions. Upon removing and reapplying the alternating electric field, the particles are able to randomize their orientation, so that their direction of circling may change randomly. A representative example of the switch in the direction of the circling is depicted in figure 5.5. In this figure, circling motion in the counterclockwise direction is represented in red, circling motion in the clockwise direction is represented in blue and the observed translational Brownian motion in the absence of an alternating electric field is represented in black. Here, the alternating electric field was switched on- and off in 20 second intervals.

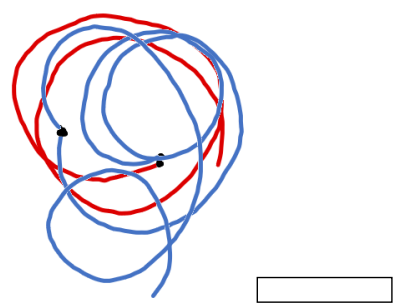

Figure 5.5: A representative trajectory of an active colloidal circler, prepared by sputter coating its monolayer under a glancing angle of $\theta=40^{\circ}$ with a gold, where the alternating electric field was turned on- and off in 20 second intervals. Its counterclockwise circling motion is represented in red, its clockwise circling motion in blue and, with the alternating electric field turned off, its Brownian motion in black. The trajectory starts with the active colloidal circler circling in the anticlockwise direction (red). When turned on, the alternating electric field was set at a magnitude of $E_{p t p}=120 \mathrm{~V} / \mathrm{mm}$ and frequency of $f=3 \mathrm{kHz}$. The scale bar represents $50 \mu \mathrm{m}$.

While we did not study the time scales required for the particles to randomize their direction of circling in detail, an off-time of 20 seconds was observed to be enough for the particles to significantly relax and randomize their direction of circling. Due to the high density of gold, the weight distribution of these active colloids is very asymmetric. As a result, upon removing the applied alternating electric field, these active colloids quickly reorient such that their gold patch faces downwards. Finally, we note that in the earlier work of Archer et al., where similar colloids were studied using hydrogen peroxide as a fuel source, a continuous switching was observed in their direction of circling [6]. Here, the active colloids were not pinned which allowed the Brownian rotational motion to rotate the particles freely along the $x$-, $y$ - and $z$-axes. 


\subsubsection{Tuning the particles' active circling behaviour in situ}

We continue in this subsection by studying the influence of the magnitude $E_{p t p}$ and frequency $f$ of the applied alternating electric field on the behaviour of the active colloidal circlers. Here, the active colloidal circlers were prepared by coating their monolayers under a glancing angle of $\theta=40^{\circ}$ with gold, which are the particles that circle in the smallest radii as described in the previous subsection. Representative trajectories of an active colloidal circler under the influence of an alternating electric field with a magnitude of $E_{p t p}=$ $120 \mathrm{~V} / \mathrm{mm}$ and frequency of $f=3,4,5$ and $6 \mathrm{kHz}$ are depicted in figure 5.6a. Alternatively, representative trajectories of active colloidal circler under the influence of an alternating electric field with a magnitude of $E_{p t p}=40$, 80 and $120 \mathrm{~V} / \mathrm{mm}$ and a frequency of $f=3 \mathrm{kHz}$ are depicted in figure $5.6 \mathrm{~b}$. The trends observed in both their velocity and angular velocity upon varying both the magnitude and frequency of the applied alternating electric field are depicted in figure 5.6c.
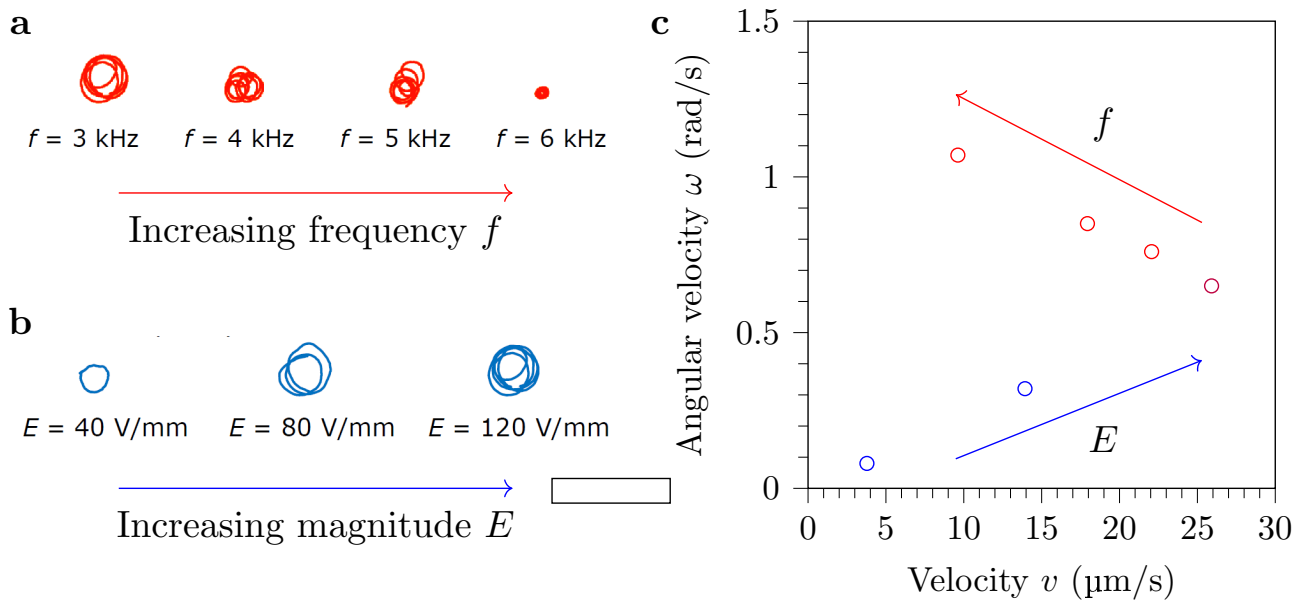

Figure 5.6: Evolution in the trajectories of an active colloidal circler prepared by sputter coating their monolayer with gold under a glancing angle of $\theta=40^{\circ}$ upon varying the frequency at a constant magnitude of $120 \mathrm{~V} / \mathrm{mm}$ (a) and upon varying the magnitude at a constant frequency of $f=3 \mathrm{kHz}(\mathrm{b})$. The trends in their velocity and angular velocity are plotted in (c). The scale bar for (a) and (b) represents $100 \mu \mathrm{m}$.

In figure 5.6a it can clearly be seen that the radii of the circular trajectories decrease upon increasing the frequency of the applied alternating electric field. From the graph in figure 5.6c it becomes clear that, upon increasing the frequency, the velocity decreases and the angular velocity increases. Since the radii of circling is determined as $r=v / \omega$, the angular velocity increases more strongly than the velocity decreases. Upon increasing the magnitude of the 
applied alternating electric field, however, no significant change in the radii can be observed in figure 5.6b. This also follows from the graph depicted in figure $5.6 \mathrm{c}$, where the points follow a straight line though the origin. Thus, upon increasing the magnitude of the applied alternating electric field, the velocity and angular velocity increase equally strong.

The average velocity and angular velocity of the active colloidal circlers as a function of the magnitude of the applied alternating electric field at a constant frequency of $f=3 \mathrm{kHz}$ are depicted in figure $5.7 \mathrm{a}$ and $\mathrm{b}$ respectively. In figure $5.7 \mathrm{a}$ and $\mathrm{b}$ it can be seen that both the velocity and angular velocity of the active colloidal circlers increases quadratically upon increasing the magnitude of the alternating electric field. The quadratic scaling for their velocity is as expected for active colloids self-propelled by ICEP [16]. Alternatively, the average velocity and angular velocity of the active colloidal circlers as a function of the frequency of the applied alternating electric field at a constant magnitude of $E_{p t p}=120 \mathrm{~V} / \mathrm{mm}$ are depicted in figure $5.7 \mathrm{c}$ and d respectively. Here, it can be confirmed that the decrease in the radii of the circling motion, which occurs upon increasing the frequency of the applied alternating electric field, is mainly due to a reduced velocity, as their angular velocity only increases slightly. While not shown here, we found that upon further increasing the frequency of the applied alternating electric fields the particles velocity slowed down further and completely stopped at a frequency of around $10 \mathrm{kHz}$. Upon further increasing the frequency, the particles were observed to begin to propel in their backwards direction, now with their gold patch facing forward. This is known behaviour of metal coated polymer Janus colloids self-propelled by ICEP [11]. Beyond this cross-over frequency, however, the particles did not display any circling motion and instead only self-propelled into straight trajectories. The active behaviour of these particles was hence not studied in this frequency range. The frequency range in which these active colloids display circling behaviour is hence limited in the range of around $f=1$ to $10 \mathrm{kHz}$.

We note that, since the translation and rotational Brownian motion does not depend on the applied alternating electric field, the influence of both types of Brownian motion is generally more significant on the trajectories with low velocities, which makes their circling motion less well-defined. Due to these particles' relatively large diameter, the circling motion is still well-defined even at a low magnitude of the applied alternating electric field as seen in the trajectory depicted in figure 5.6b. This may not be true for circling active colloids with smaller diameters.

Thus, both the magnitude and frequency of the applied alternating electric field influence both the velocity and angular velocity of the active colloidal circlers at the same time. By varying both the frequency and magnitude, the active colloids can therefore be made to circle with a certain velocity and a certain angular velocity, which may make these active colloids very 

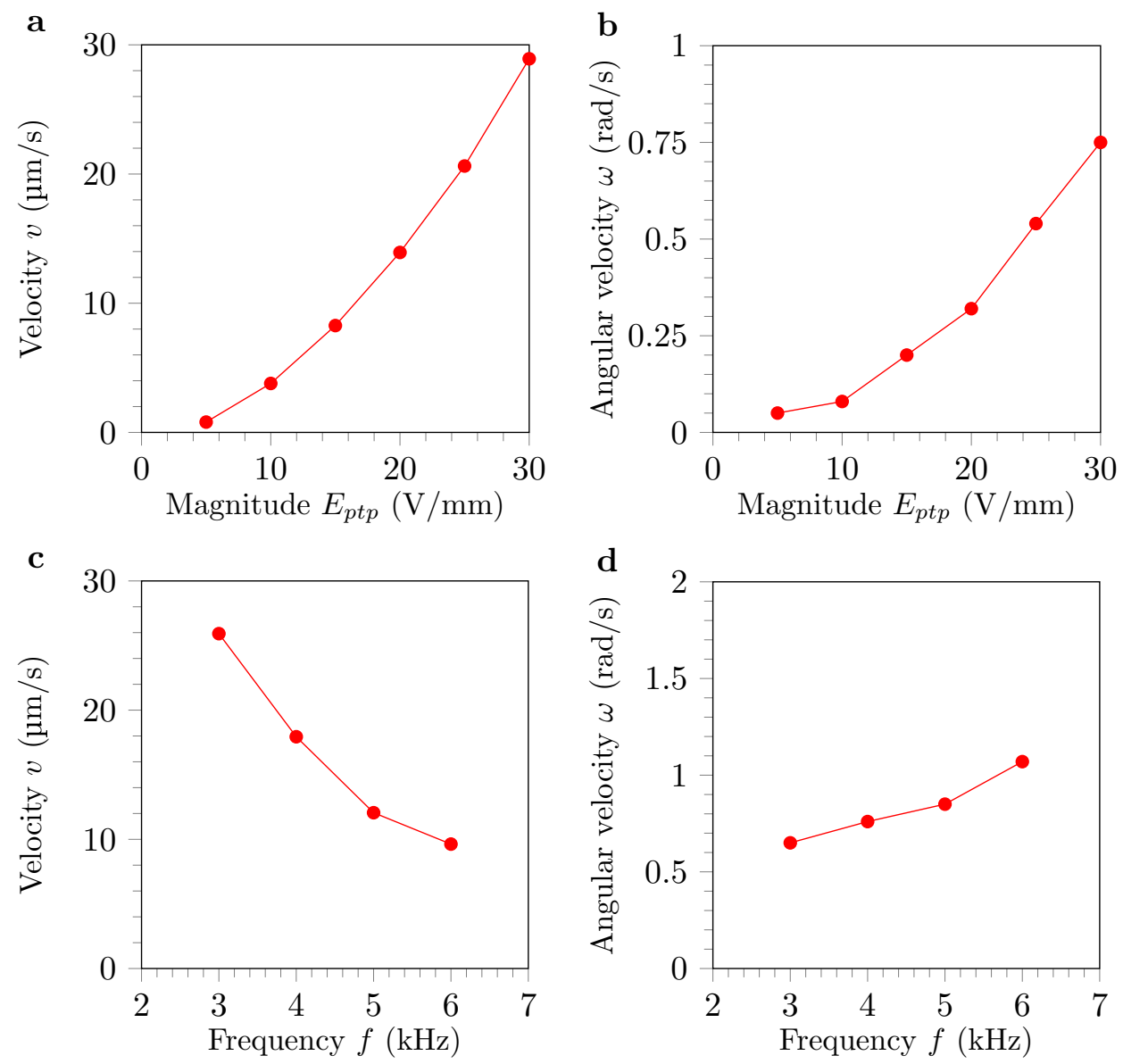

Figure 5.7: The velocity (a) and angular velocity (b) of an active colloidal circler as a function of the magnitude of the applied alternating electric field at a constant frequency of $3 \mathrm{kHz}$, and the velocity (c) and angular velocity (d) as a function of the frequency of the applied alternating electric field at a constant magnitude of $120 \mathrm{~V} \mathrm{~mm}^{-1}$ for active colloidal circlers prepared by sputter coating their monolayer under a glancing angle of $\theta=40^{\circ}$.

interesting for self-assembly studies since their behaviour can be fully tuned in situ very easily. Furthermore, since a certain combination of velocity and angular velocity thus corresponds to a certain magnitude and frequency of the applied alternating electric field, these active colloidal circlers may be suitable for sensors where they can measure the both the magnitude and frequency of the alternating electric field simultaneously.

\subsubsection{Further reducing the remaining the translational motion}

In addition to the active colloidal circlers described in the previous two subsections, we here present a modification in the design of these type of active 
colloids in order to further reduce their remaining translational motion and velocity. By introducing a second metallic patch on the opposite side of the particle, colloidal particles with inversion symmetry can be obtained. The two patches should therefore cancel out each others induced translational motion, while amplifying each others induced rotational motion. These so-called active colloidal spinners were studied under the influence of an applied alternating electric field with a magnitude of $E_{p t p}=80 \mathrm{~V} / \mathrm{mm}$ and frequency of $10 \mathrm{kHz}$. In practice, however, we observed that these active colloidal spinners still displayed small amounts of translational motion and a thus a small velocity. Since the two platinum patches could not be distinguished easily using optical microscopy in order to track the rotational motion of the particles, we have used this small amount of remaining translational motion to determine the rotational speed of these particles instead. Similarly to the active colloidal circlers described in the previous subsections, we first determined the mean squared displacement of these type of active colloids using equation 5.1, and subsequently determined the velocity and angular velocity of the particles by fitting the temporal evolution of the mean squared displacement as described in equation 5.2. A representative example of the mean squared displacement along with its fit of an active colloidal spinners is depicted in figure 5.8. It can be seen that the fit agrees very well with the experimental data. For this particular particle, the velocity and angular velocity were determined to be $v=0.69 \mathrm{\mu m} / \mathrm{s}$ and $\omega=0.55 \mathrm{rad} / \mathrm{s}$ respectively. Similar to the active colloidal circlers, switches in the direction of rotation were not observed as long as the alternating electric field was applied, resulting in these particles also being effectively chiral in two dimensions.

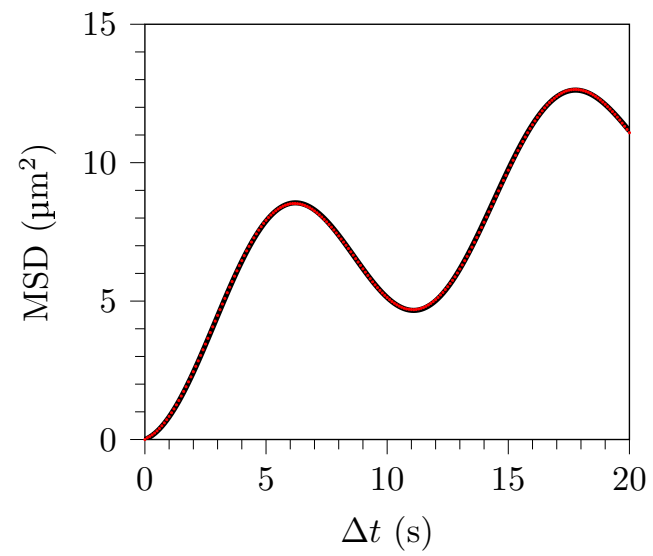

Figure 5.8: The mean squared displacement (red marks) of an active colloidal spinner studied under the influence of an alternating electric field of magnitude $E_{p t p}=80 \mathrm{~V} / \mathrm{mm}$ and frequency $f=10 \mathrm{kHz}$ along with their fits (black line).

By determining the velocity and angular velocity of 25 different active colloidal spinners, we have determined their average velocity and angular velocity 
to be $v=1.54 \pm 69 \% \mu \mathrm{m} / \mathrm{s}$ and $\omega=0.60 \mathrm{rad} / \mathrm{s} \pm 70 \%$ respectively. The average radii in which these active colloidal spinners circle is therefore $r=2.6 \mu \mathrm{m}$, which is similar to the diameter of the particles and approximately one order of magnitude smaller than the active colloidal criclers prepared at the highest glancing angle, while their angular velocities are comparable. It can be concluded, however, that the rotational speed of these active colloidal spinners is rather low, as this average angular velocity corresponds only to a full rotation approximately every $10 \mathrm{~s}$. An increase in the magnitude of the applied alternating electric field, however, should further increase their rotational speed. Furthermore, the spread in the angular velocities is relatively high compared to active colloidal circlers. The increased spread in these properties is possibly a result of the more complicated synthesis process, which increases the chances for deviations to occur in the shape of the metallic patches.

Finally, we note that the degree in which both of these types of active colloids circle and spin might be improved upon by improving the syntheses processes with which these particles are prepared. The use of a more directional method for the metal deposition might increase the asymmetry of the active colloids, therefore making their characteristic properties more pronounced. This may result in both the active colloidal circlers and spinners having a higher angular velocity, thus displaying circular motion in smaller radii.

\subsection{Conclusion}

By varying the glancing angle at which colloidal monolayers are sputter coated, the asymmetry of the resulting metal patch can be controlled. In this work we have prepared active colloids by first assembling large PMMA colloids into monolayers using the unidirectional rubbing method, and subsequently sputter coating these with $15 \mathrm{~nm}$ layer of gold. By sputter coating them under a glancing angle of $\theta=0^{\circ}$, a symmetrical patch can be introduced, and the resulting colloid were observed to be self-propelled with straight trajectories under the influence of an applied alternating electric field. When sputter coated under a glancing angle of $\theta>0^{\circ}$ instead, colloids with an asymmetrical patch can be prepared which were observed to self-propel into circular trajectories. Here, the average radii of the circling motion was observed to decrease upon increasing the glancing angle at which the colloidal monolayers were sputter coated. For all active colloidal circlers, we observed that the spread in their velocity and angular velocity was relatively small, up to a maximum of around 20 and $30 \%$ respectively. Furthermore, it was observed that the velocity and the angular velocity of the active colloidal circlers increased equally and quadratically upon increasing the magnitude of the alternating electric field, thus keeping the radii of circling constant. Upon increasing the 
frequency of the alternating electric field, however, the velocity decreased but the angular velocity increased more strongly, resulting in circular motion with smaller radii. Due to the metallic patch being pinned in the direction of the applied alternating electric field, these active colloidal circlers were effectively chiral in two dimensions. Their orientation, and therefore their chirality, can be randomized turning off and on the alternating electric field for long enough intervals.

The tunable behaviour of these active colloidal circlers, long with the relatively small spread in their behaviour, makes them interesting candidates for future self-assembly studies where their behaviour might result in the formation of novel active phases. Furthermore, their response to an applied alternating electric field may make these active colloids suitable for usage in sensors, where they might be used to measure both the magnitude and frequency of the applied alternating electric field simultaneously.

In addition, we showed that so-called active colloidal spinners can be prepared by applying a second coating on the opposite side of the particle. This was done by coating colloidal monolayers with a thin layer of platinum under a glancing angle, transferring the colloidal monolayer to a much more sticky PDMS-coated surface and subsequently sputter coating the monolayer again under the same glancing angle. The resulting particles have inversion symmetry and rotate in place under the influence of an applied alternating electric field with only minor amounts of translational motion. These type of spinners might be usable to guide various self-assembly processes.

\subsection{Acknowledgements}

Pepijn Moerman is thanked for providing the script to track the particles in time. Annemiek de Vries is thanked for her help with the preparation of the active colloidal circlers during her bachelor thesis. Kyra, Ffion, Friso and Sander are thanked for their help with the development of the active colloidal spinners during their second year research project.

\section{References}

[1] W. Wang, W. Duan, S. Ahmed, T. E. Mallouk, and A. Sen, "Small power: Autonomous nano- and micromotors propelled by self-generated gradients," Nano Today, vol. 8, no. 5, pp. 531-554, 2013.

[2] C. W. Shields and O. D. Velev, "The evolution of active particles: Toward externally powered self-propelling and self-reconfiguring particle systems," Chem, vol. 3, no. 4, pp. 539-559, 2017. 
[3] W. Wang, X. Lv, J. L. Moran, S. Duan, and C. Zhou, "A practical guide to active colloids: choosing synthetic model systems for soft matter physics research," Soft Matter, vol. 16, no. 16, pp. 3846-3868, 2020.

[4] F. Kümmel, B. Ten Hagen, R. Wittkowski, I. Buttinoni, R. Eichhorn, G. Volpe, H. Löwen, and C. Bechinger, "Circular motion of asymmetric self-propelling particles," Physical Review Letters, vol. 110, no. 19, pp. 1-5, 2013.

[5] A. B. Pawar and I. Kretzschmar, "Patchy particles by glancing angle deposition," Langmuir, vol. 24, no. 2, pp. 355-358, 2008.

[6] R. J. Archer, A. I. Campbell, and S. J. Ebbens, "Glancing angle metal evaporation synthesis of catalytic swimming Janus colloids with well defined angular velocity," Soft Matter, vol. 11, no. 34, pp. 6872-6880, 2015.

[7] I. Buttinoni, J. Bialké, F. Kümmel, H. Löwen, C. Bechinger, and T. Speck, "Dynamical clustering and phase separation in suspensions of self-propelled colloidal particles," Physical Review Letters, vol. 110, no. 23, pp. 1-5, 2013.

[8] J. Bialké, T. Speck, and H. Löwen, "Active colloidal suspensions: Clustering and phase behavior," Journal of Non-Crystalline Solids, vol. 407, pp. 367-375, 2015.

[9] J. Palacci, S. Sacanna, A. Abramian, J. Barral, K. Hanson, A. Y. Grosberg, D. J. Pine, and P. M. Chaikin, "Artificial rheotaxis," Science Advances, vol. 1, no. 4, pp. 1-6, 2015.

[10] G. Gonnella, D. Marenduzzo, A. Suma, and A. Tiribocchi, "Motility-induced phase separation and coarsening in active matter," Comptes Rendus Physique, vol. 16, no. 3, pp. 316-331, 2015.

[11] J. Yan, M. Han, J. Zhang, C. Xu, E. Luijten, and S. Granick, "Reconfiguring active particles by electrostatic imbalance," Nature Materials, vol. 15, no. 10, pp. 1095-1099, 2016.

[12] J. L. Aragones, J. P. Steimel, and A. Alexander-Katz, "Elasticity-induced force reversal between active spinning particles in dense passive media," Nature Communications, vol. 7, pp. 1-9, 2016.

[13] B. Van Der Meer, L. Filion, and M. Dijkstra, "Fabricating large two-dimensional single colloidal crystals by doping with active particles," Soft Matter, vol. 12, no. 14 , pp. 3406-3411, 2016.

[14] S. J. Ebbens and J. R. Howse, "In pursuit of propulsion at the nanoscale," Soft Matter, vol. 6, no. 4, pp. 726-738, 2010.

[15] S. J. Ebbens, "Active colloids: Progress and challenges towards realising autonomous applications," Current Opinion in Colloid and Interface Science, vol. 21, pp. 14-23, 2016.

[16] S. Gangwal, O. J. Cayre, M. Z. Bazant, and O. D. Velev, "Induced-charge electrophoresis of metallodielectric particles," Physical Review Letters, vol. 100, no. 5 , pp. 1-4, 2008.

[17] Q. Chen, S. C. Bae, and S. Granick, "Directed self-assembly of a colloidal kagome lattice," Nature, vol. 469, no. 7330, pp. 381-384, 2011. 


\section{Chapter 6}

\section{Production of Active Anisotropic Hydrogel Particles}

\section{Abstract}

In this chapter we present a novel method for synthesizing active anisotropic hydrogel particles. This low-cost method is easy to implement, employing any microscope, does not require cleanroom or microfluidic expertise and eliminates the need for sacrificial layers. Using an automated process, the particles are created by locally polymerizing spin coated films of poly(ethylene glycol) diacrylate (PEGDA) using UV light at a rate of up to about 10.000 particles per hour. The geometry of the resulting particles is determined by the shape of the mask placed at the field stop of the microscope, while their thickness is determined by the thickness of the spin coated film. Furthermore, we demonstrate that these particles display active motion under the influence of alternating electric fields. The behaviour of these active hydrogel particles is determined by their shape as they are driven by electrohydrodynamic (EHD) flows, which are induced by their asymmetry. This study opens the synthesis of active anisotropic hydrogel particles to a broader audience without access to cleanrooms, microfluidics or wet-chemistry know-how. 


\subsection{Introduction}

Particle shape plays an essential role in dictating both the equilibrium and out-of-equilibrium behaviour in colloidal systems [1-4]. Particularly, for active particles driven by external forces [5], chemical reactions [6] or gradients [7], it has been demonstrated that the shape of the particle determines its behaviour, both on an individual and on a collective level $[8,9]$. For example, asymmetric active L-shaped particles have been shown to swim in circular trajectories [10], while active gear-shaped particles rotated in place [11]. On a collective level these active particles have demonstrated self-assembly into various hierarchical structures such hexagonal close-packed clusters [12] or as an asymmetrical gear-shaped assemblies [13]. A broad spectrum of applications ranging from photonics [14, 15], tailored pharmaceutical matrices [16], anti-counterfeiting materials [17], drug delivery systems [18] and tissue engineering [19] can potentially benefit from the development of such active building blocks.

Wet chemistry and lithographic techniques are the most common used approaches for producing shape anisotropic particles. While wet chemical synthesis methods can produce large quantities on the order of grams to kilograms, they are limited in the geometries they can offer. So far, only rather simple shapes such as spheres, cubes [20], rods [21] or dumbbell-shaped particles [21] can usually be produced by wet chemistry. On the other hand, lithographic techniques are able to provide precise control over the particle shape, although at much lower yields. For example, stop-flow lithography can be used but it is limited to produce particles with out-of-plane symmetry [2224]. Using special techniques such as PRINT [25], two-photon lithography [26] or $3 \mathrm{D}$ printing $[27,28]$, particles with more asymmetry can be produced and at a higher resolution. Furthermore, photolithography can be used to produce particles but often requires the use of a sacrificial layer $[29,30]$. These methods are often complicated and may require extensive synthesis knowledge and access to cleanrooms.

In this chapter we describe a novel method that allows the production of anisotropic hydrogel particles at a rate of up to about 10.000 particles per hour with dimensions as small as $10 \mu \mathrm{m}$. This method relies exclusively on inexpensive and readily available starting materials. Moreover, these anisotropic hydrogel particles can be produced without the use of a cleanroom or dedicated lithography equipment. These particles, depending on their shape, display active motion under the influence of an alternating electric field. We briefly show the active behaviour of some of these active anisotropic hydrogel particles that become self-propelled due to the presence of electrohydrodynamic (EDH) flows [31-33]. These active anisotropic hydrogel particles are promising for self-assembly studies and for the development of novel materials. 


\subsection{Experimental methods}

\subsubsection{Preparation of the anisotropic hydrogel particles}

Glass slides (\#0, $60 \mathrm{~mm} \times 24 \mathrm{~mm} \times 0.085-0.12 \mathrm{~mm})$ were immersed in Piranha solution containing sulfuric acid (Sigma Aldrich, $\geq 95 \%$ ) and hydrogen peroxide (Sigma Aldrich, 30\%) in a ratio of 3:1 by volume for 30 minutes in order to both clean the glass surface and to increase its hydrophilicity. They were rinsed afterwards with water (Milli-Q, $18.2 \mathrm{M} \Omega \cdot \mathrm{cm}$ ) and dried under a nitrogen flow. The glass slides were then spin coated with a film of poly(ethylene glycol)diacrylate (PEGDA, Sigma Aldrich, $M_{w}=700 \mathrm{~g} / \mathrm{mol}$ ) at $1000 \mathrm{rpm}$ for various durations. As a result of the hydrophilization the spin coated PEGDA film is stable for several hours. Here, the PEGDA contained 5 wt.\% of the dissolved photoinitiator 2-hydroxy-2-methylpropiophenone (Sigma). The hydrogel particles were then printed one by one using a Nikon Eclipse Ti-U optical microscope equipped with a motorized stage, a R405 filter and a $\mathrm{Hg}$ lamp coupled to a fast shutter. In this setup, the filter directed the UV light to the sample, while allowing light of higher wavelengths to pass to the camera for regular imaging. The focal point of the UV light was kept halfway inside the PEGDA film during the printing of the particles in order to obtain particles with as much out-of-plane symmetry as possible. Using an illumination time of $50 \mathrm{~ms}$, a yield of about 10.000 particles per hour resulted. A mask containing a single projection was in each of the experiments described here. Masks containing multiple projections, however, can be used to increase the production rate of the hydrogel particles. The unpolymerized PEGDA was removed afterwards by rinsing the glass slides carefully with ethanol. The glass slides were then suspended in water and ultrasonicated for 10 minutes in order to release the resulting particles from the glass substrate and to disperse them into solution. The production process is schematically depicted in figure 6.1 .

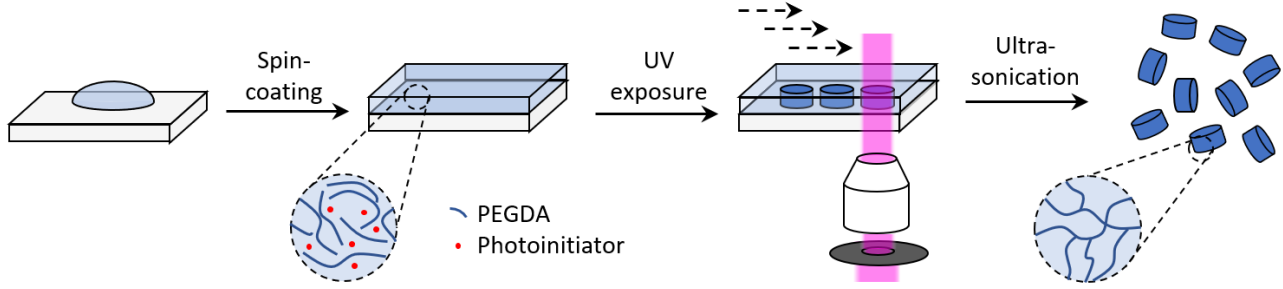

Figure 6.1: A schematic illustration of the production process of the anisotropic hydrogel particles. PEGDA containing the photoinitiator is first spin coated onto hydrophilized glass. The hydrophilization step with Piranha solution ensures the formation of a homogeneous film during spin coating. The resulting PEGDA film is then sequentially illuminated with UV light through a mask, thus creating polymerized particles in a raster. Afterwards, the glass slide is ultrasonicated to release the polymerized particles from the glass surface and to disperse them into solution. 


\subsubsection{Analysis of their active motion}

To study their active motion, the anisotropic hydrogel particles were first dispersed in an aqueous solution containing $0.1 \mathrm{~g} / \mathrm{L}$ Pluronic F127. Small volumes of these dispersions were then used to fill the custom-made cells consisting of two ITO-coated glass slides spaced $100 \mu \mathrm{m}$ apart with their conductive ITOcoated surfaces on the inside of the cell and in direct contact with the dispersion as described in section 2.5. The surfactant Pluronic F127 was added to prevent the particles from sticking to the ITO-coated surfaces. An alternating electric field perpendicular to the field of view was applied between the ITO-coated surfaces using a Yokogawa FG120 function generator coupled to an Ohmtronics 7602M wideband amplifier. A Yokogawa DLM2022 digital oscilloscope was used to determine the amplitude of the applied alternating electric field. The active hydrogel particles were imaged using a Nikon Eclipse Ti-U optical microscope equipped with a Nikon 10x objective. A frame rate of 10 fps was used for all samples studied. A custom-written Matlab script was used in order to track the particles in time.

\subsection{Results and discussion}

\subsubsection{Adjusting the in-plane geometry of the particles}

An image of the rinsed glass slide with the printed hydrogel particles still attached to the glass surface in the ordered array they were printed in is depicted in figure 6.2a. This shows that the adhesion of the printed particles to the glass substrate is strong enough for the unpolymerized PEDGA to be rinsed away without disturbing the particles. This, in addition, also opens up possibilities for further modifications of the particles such as the addition of secondary layers or directed sputter coating to further increase the anisotropy of the particles in the future. The particles could be released from the substrate easily, however, using ultrasonication in order to disperse them into solution. The inplane shape and size of these hydrogel particles is determined by the image of the mask that is installed at the field stop of the microscope. In addition, the size of the particles can be scaled by varying the magnification of the objective used. Here, the use of an objective with a higher magnification results in the production of smaller particles. Hydrogel particles produced using various masks are depicted in figure $6.2 \mathrm{~b}-\mathrm{e}$. The masks used are shown in the insets of the corresponding images. It can be seen that, although the outlines in the masks have sharp edges and square corners, the printed hydrogel particles have rounded corners. This is especially obvious for particles containing sharp corners, such as the pentagon- and moon-shaped particles, whereas it is less noticeable for particles that only have curves such as the disk-shaped particles. The observed roundedness is caused by the molecular diffusion of the PEGDA 
and photoinitiator and by the limited resolution of the optical microscope. In order to determine the smallest particle size this method can produce, we systematically decreased the magnification and identified the smallest particle we can synthesize. We have found that this method is able to produce particles in a broad size range, with dimensions starting at about $10 \mu \mathrm{m}$. Below this size, the produced hydrogel particles did not have a well-defined geometry, possibly due to the difficulty to accurately focus objectives with higher magnifications. We have found no evidence of particle shrinkage nor swelling in our experiments, as their size did not change after dispersing these particles in water. This observation is not surprising as polymerized PEGDA is very densely crosslinked. In our particular case, using of a mask with a circular hole $1 \mathrm{~mm}$ in diameter, we obtained a disk-shaped particle with a diameter of $40 \mu \mathrm{m}$ using an objective with a 60x magnification. The resulting disk-shaped particle had an in-plane aspect ratio of 1 , which shows that the UV light beam is not significantly distorted.
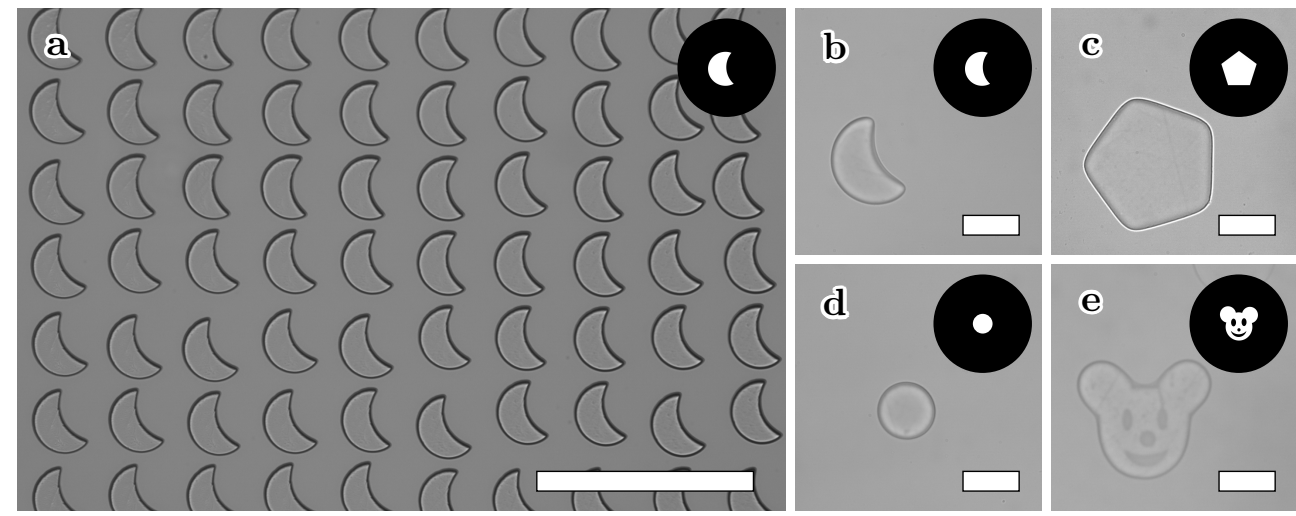

Figure 6.2: The hydrogel particles are printed one by one in an ordered array prior to releasing them from the glass surface using ultrasonication (a). The shape of the resulting particle is determined by the mask that is installed at the field stop of the microscope (b-e). All particles were prepared by spin coating at a speed of $1000 \mathrm{rpm}$ for 30 seconds. The scale bars represent $100 \mu \mathrm{m}$ (a) and $50 \mu \mathrm{m}$ (b-e).

\subsubsection{Adjusting the out-of-plane geometry of the particles}

In contrast to the in-plane dimensions, which are determined by the shape and size of the installed mask and the magnification of the objective used, the out-of-plane dimension of the particles is determined by the thickness of the PEGDA film containing the photoinitiator that is spin coated onto the piranha-treated glass. The thickness of spin coated films can be tuned by adjusting either the spin coating speed or duration. It is well-known that spin coating for longer durations and/or increasing the speed of spin coating decreases the thickness of the resulting films. In order to determine the thickness 
of the printed hydrogel particles, we have prepared particles with relatively small in-plane dimensions from PEGDA films with various thicknesses. These films were prepared by spin coating the PEDGA for various durations while keeping the spin coating speed constant at $1000 \mathrm{rpm}$. These particles were prepared using a mask with a small circular hole. As a result of their relatively small in-plane size, and thus their relatively large aspect ratio $p=h / d>1$, where $h$ is their thickness and $d$ is their diameter, these particles lie on their side when dispersed into solution, which allows their thickness to be conveniently measured. Two examples of such particles prepared from films that were spin coated at $1000 \mathrm{rpm}$ for 5 and 40 seconds are depicted in figure 6.3a and $\mathrm{b}$ respectively. Here, it can be seen that the spin coating time indeed allows the thickness of the particles to be tuned. It addition, it can be seen here that the top of the particles are rounded. The corners where the particles were attached to the glass surface, however, have a more square-like shape. This may result from the oxygen present in air, which quenches the radical polymerization near the exposed top of the spin coated PEGDA film.

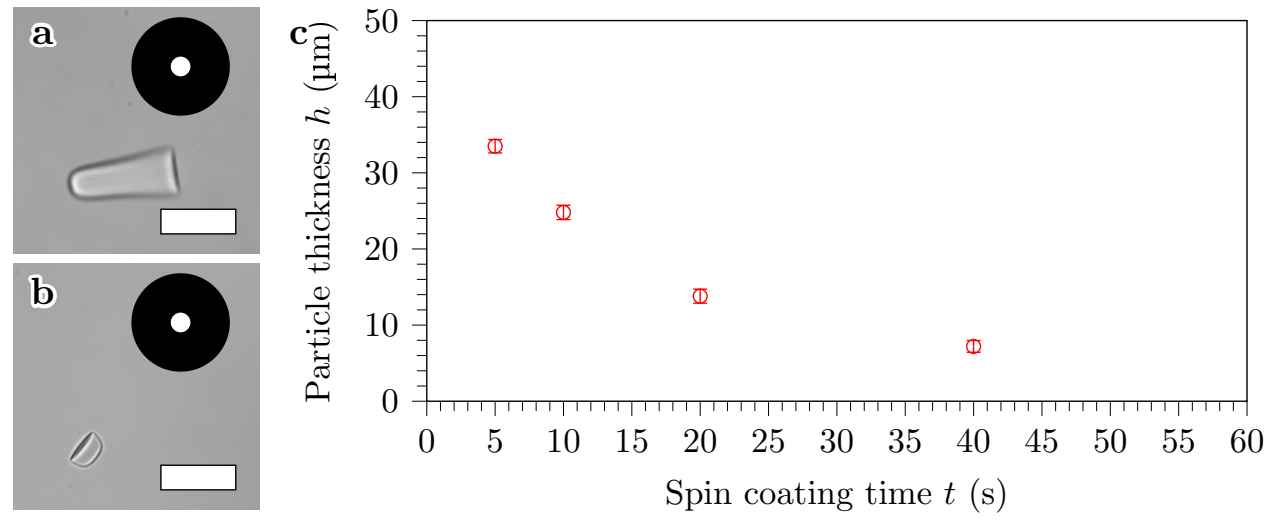

Figure 6.3: Hydrogel particles with an aspect ratio of $p=h / d>1$ lie on their sides when dispersed in solution ( $\mathrm{a}$ and $\mathrm{b}$ ). These particles were prepared by spin coating their PEGDA films at a speed of $1000 \mathrm{rpm}$ for 5 (a) and 40 (b) seconds. This allows the thickness $h$ of the resulting hydrogel particles to be conveniently measured. The average thickness $h$ and its standard deviation $\sigma_{h}$ of these hydrogel particles were determined as a function of the spin coating duration at a constant speed of $1000 \mathrm{rpm}$ (c). The scale bars in (a) and (b) represent $25 \mu \mathrm{m}$.

The thickness of the resulting particles were measured for various spin coating durations at a constant speed of $1000 \mathrm{rpm}$. These thicknesses, along with their standard deviations, are depicted in figure 6.3c. For each of the samples, the standard deviation in the thickness of the particles was around $\sigma_{h}=1 \mu \mathrm{m}$. This resulted in a polydispersity of $2.6,3.8,6.7$, and $10.6 \%$ in the thickness of the hydrogel particles prepared at a spin coating time of $t=5,10$, 20 and 40 s respectively. Finally, we note that particles with a larger thickness may be prepared by decreasing the spin coating speed, while particles with a 
smaller thickness may be prepared by increasing the spin coating speed or by diluting the PEGDA photoinitiator mixture with a suitable, volatile solvent.

\subsubsection{The active motion of anisotropic hydrogel particles}

In this subsection we briefly describe the active motion we have observed for these active hydrogel particles. In order to study the effect of the shape of the anisotropic hydrogel particles on their active behaviour, we have prepared particles with various shapes and studied their active motion under the influence of an applied alternating electric field. We found that asymmetrical dumbbell-shaped particles approximately $20 \mu \mathrm{m}$ in length displayed an active motion in the direction of their largest lobe. While most of these particles were self-propelled into relatively straightforward directions, a significant fraction of these asymmetrical dumbbell-shaped hydrogel particles displayed active motion in circular trajectories instead. An example of an asymmetrical dumbbell-shaped particle with self-propulsion in a circular trajectory is depicted in figure 6.4a. Similarly, cylinder-shaped hydrogel particles with an aspect ratio of $p>1$, which lie on their sides in solution as shown previously in figure 6.3a, obtain an in-plane anisotropic shape as one end of the particle is rounded while the other end has sharp corners. These particles approximately $10 \mu \mathrm{m}$ in length were also observed to be self-propelled in the direction of their largest lobe, and, in addition, also displayed various degree of circling. Examples of the these cylinder-shaped hydrogel particles and their trajectories are depicted in figure $6.4 \mathrm{~b}$. It can be seen here that the three particles show various degree of circling in their trajectories.
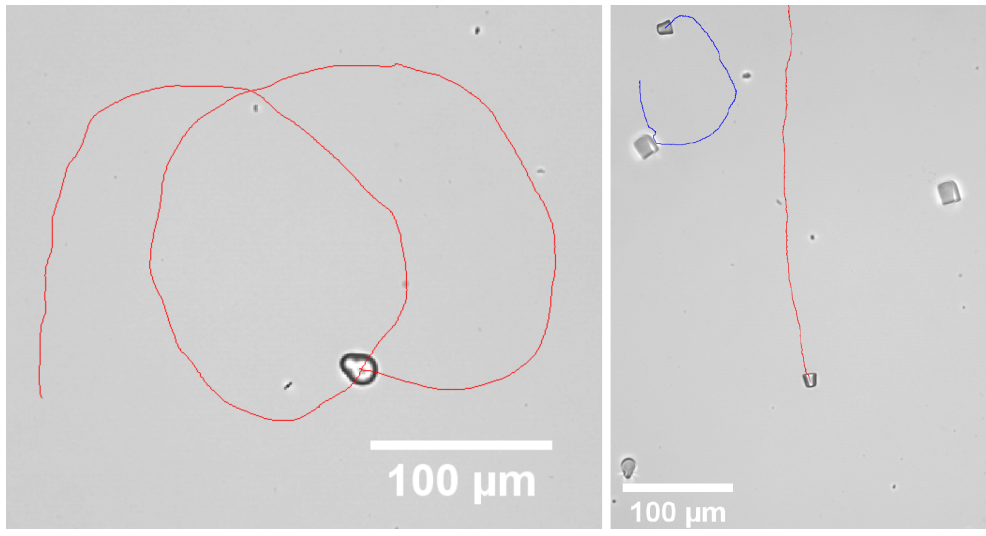

Figure 6.4: Examples of the active motion of the hydrogel particles under the influence of an applied alternating electric field. The images show the active hydrogel particles in their initial position and show the trajectory in which they will propel themselves. The applied alternating electric field had a magnitude of $E_{p t p}=80 \mathrm{~V} / \mathrm{mm}$ (a) and $120 \mathrm{~V} / \mathrm{mm}$ (b) and frequency of $f=1 \mathrm{kHz}$. 
Despite the addition of the surfactant Pluronic F127, these hydrogel particles often became stuck to the ITO-coated surface, especially the larger hydrogel particles and under the influence of relatively strong alternating electric fields. As a result, unfortunately, the behaviour of these active hydrogel particles could not be determined accurately. Generally, however, the velocity of the particles described in this subsection were around $1 \mu \mathrm{m} / \mathrm{s}$, depending on the magnetude and frequency of the applied alternating electric field. These particles become active under the influence of alternating electric fields due to the presence of electrohydrodynamic $(\mathrm{EDH})$ flows induced around the particles surface [31]. For particles with an anisotropic geometry, these flows will generate a net motion of the particle. The observation that these asymmetric dumbbell-shaped hydrogel particles self-propelled themselves with their largest lobe facing forward are in agreement with the known literature [32, 33]. The observation that they displayed various degrees of circling might be due to deviations in the shape of these hydrogel particles caused by experimental imperfections. Defects in the geometry of active particles are known to result in circular trajectories, especially for self-propulsion mechanisms using alternating electric fields [34]. While we have shown here that these hydrogel particles can be made active, it is clear that more research is required in order to make any strong conclusions on their active behaviour. Possibly, changes in the synthesis procedure of these hydrogel particles might result in their active motion to be more well-defined. In addition, by further increasing the anisotropy in the shape of the particles it may possible to obtain particles that swim in circular trajectories similar to the L-shaped active particles mentioned previously [10].

\subsection{Conclusion}

We have developed a novel, low-cost method for the production of anisotropic hydrogel particles. This methods is easy to implement using any microscope equipped with an UV light source and an motorized microscope stage and does not require cleanroom or microfluidic expertise. In this method, films of PEGDA are spin coated on piranha treated hydrophilic glass surfaces and locally crosslinked using UV light. This allows the production of hydrogel particles as small as $10 \mu \mathrm{m}$ in size at a rate of up to 10.000 particles per hour. The in-plane shape and size of the resulting hydrogel particles are determined by the mask that is installed at the field stop of the microscope and the magnification of the objective used. Their thickness and out-of-plane geometry is determined by the thickness of the spin coated PEGDA film. Using two types of anisotropic hydrogel particles we have briefly shown that these hydrogel particles display active motion under the influence of an applied alternating electric field. We found, however, large variations in the behaviour of these 
active hydrogel particles with various degrees of circling motion, possibly due to defects in the geometry of these particles. Further research into the behaviour and implementation of this synthesis method is therefore required to understand the behaviour of these active anisotropic hydrogel particles. In the future these active particles can potentially be used for the active self-assembly of active particles with arbitrary shapes. We believe the proposed method can open new alleys of investigation of active particles with applications in drug delivery, tissue engineering, bottom-up manufacturing and beyond.

\subsection{Acknowledgments}

Kasper Tempel is thanked for his help with the synthesis of the hydrogel particles and for his help studying their active motion. Florine Rombach is thanked for her help with studying the active motion of these particles. Pepijn Moerman is thanked for providing the script that was used to track the particles. Burak Eral is thanked for the fruitful discussions and for his help with the writing.

\section{References}

[1] G. M. Whitesides and M. Boncheva, "Supramolecular chemistry and self-assembly special feature: Beyond molecules: Self-assembly of mesoscopic and macroscopic components," Proceedings of the National Academy of Sciences of the United States of America, vol. 99, no. 8, p. 4769, 2002.

[2] V. N. Manoharan, "Colloidal matter: Packing, geometry, and entropy," Science, vol. 349, no. 6251, 2015.

[3] S. Sacanna, D. J. Pine, and G. R. Yi, "Engineering shape: The novel geometries of colloidal self-assembly," Soft Matter, vol. 9, no. 34, pp. 8096-8106, 2013.

[4] F. Li, D. P. Josephson, and A. Stein, "Colloidal assembly: The road from particles to colloidal molecules and crystals," Angewandte Chemie - International Edition, vol. 50, no. 2, pp. 360-388, 2011.

[5] H. Löwen, "Colloidal dispersions in external fields: Recent developments," Journal of Physics Condensed Matter, vol. 20, no. 40, 2008.

[6] W. E. Uspal, "Theory of light-activated catalytic Janus particles," Journal of Chemical Physics, vol. 150, no. 11, 2019.

[7] J. L. Moran and J. D. Posner, "Phoretic self-propulsion," Annual Review of Fluid Mechanics, vol. 49, no. 1, pp. 511-540, 2017.

[8] D. Dendukuri and P. S. Doyle, "The synthesis and assembly of polymeric microparticles using microfluidics," Advanced Materials, vol. 21, no. 41, pp. 40714086, 2009. 
[9] M. E. Helgeson, S. C. Chapin, and P. S. Doyle, "Hydrogel microparticles from lithographic processes: Novel materials for fundamental and applied colloid science," Current Opinion in Colloid and Interface Science, vol. 16, no. 2, pp. 106$117,2011$.

[10] F. Kümmel, B. Ten Hagen, R. Wittkowski, I. Buttinoni, R. Eichhorn, G. Volpe, H. Löwen, and C. Bechinger, "Circular motion of asymmetric self-propelling particles," Physical Review Letters, vol. 110, no. 19, pp. 1-5, 2013.

[11] C. Maggi, J. Simmchen, F. Saglimbeni, J. Katuri, M. Dipalo, F. De Angelis, S. Sanchez, and R. Di Leonardo, "Self-Assembly of Micromachining Systems Powered by Janus Micromotors," Small, vol. 12, no. 4, pp. 446-451, 2016.

[12] J. Palacci, S. Sacanna, A. P. Steinberg, D. J. Pine, and P. M. Chaikin, "Living crystals of light-activated colloidal surfers," Science, vol. 339, no. 6122, pp. 936940, 2013.

[13] A. Aubret, M. Youssef, S. Sacanna, and J. Palacci, "Targeted assembly and synchronization of self-spinning microgears," Nature Physics, vol. 14, no. 11, pp. 1114-1118, 2018.

[14] A. C. Arsenault, D. P. Puzzo, I. Manners, and G. A. Ozin, "Photonic-crystal full-colour displays," Nature Photonics, vol. 1, no. 8, pp. 468-472, 2007.

[15] Y. Liu, L. Shang, H. Wang, H. Zhang, M. Zou, and Y. Zhao, "Multicolored photonic barcodes from dynamic micromolding," Materials Horizons, vol. 5, no. 5, pp. 979-983, 2018.

[16] H. B. Eral, M. O’Mahony, R. Shaw, B. L. Trout, A. S. Myerson, and P. S. Doyle, "Composite hydrogels laden with crystalline active pharmaceutical ingredients of controlled size and loading," Chemistry of Materials, vol. 26, no. 21, pp. 62136220, 2014.

[17] I. Rehor, S. van Vreeswijk, T. Vermonden, W. E. Hennink, W. K. Kegel, and H. B. Eral, "Biodegradable microparticles for simultaneous detection of counterfeit and deteriorated edible products," Small, vol. 13, no. 39, pp. 1-9, 2017.

[18] U. A. Gurkan, S. Tasoglu, D. Kavaz, M. C. Demirel, and U. Demirci, "Emerging technologies for assembly of microscale hydrogels," Advanced Healthcare Materials, vol. 1, no. 2, pp. 149-158, 2012.

[19] K. D. Higgins, S. C. Benjamin, T. M. Stace, G. J. Milburn, B. W. Lovett, and E. M. Gauger, "Superabsorption of light via quantum engineering," Nature Communications, vol. 5, pp. 1-7, 2014.

[20] T. Sugimoto and T. Sakata, "Preparation of monodisperse pseudocubic $\alpha$ Fe2O3 particles from condensed ferric hydroxide gel," Journal of Colloid And Interface Science, vol. 152, no. 2, pp. 587-590, 1992.

[21] P. M. Johnson, C. M. Van Kats, and A. Van Blaaderen, "Synthesis of colloidal silica dumbbells," Langmuir, vol. 21, no. 24, pp. 11 510-11 517, 2005.

[22] D. Dendukuri, D. C. Pregibon, J. Collins, T. A. Hatton, and P. S. Doyle, "Continuous-flow lithography for high-throughput microparticle synthesis," $N a$ ture Materials, vol. 5, no. 5, pp. 365-369, 2006. 
[23] H. Z. An, E. R. Safai, H. Burak Eral, and P. S. Doyle, "Synthesis of biomimetic oxygen-carrying compartmentalized microparticles using flow lithography," Lab on a Chip, vol. 13, no. 24, pp. 4765-4774, 2013.

[24] H. Z. An, H. B. Eral, L. Chen, M. B. Chen, and P. S. Doyle, "Synthesis of colloidal microgels using oxygen-controlled flow lithography," Soft Matter, vol. 10, no. 38, pp. 7595-7605, 2014.

[25] S. E. Gratton, P. D. Pohlhaus, J. Lee, J. Guo, M. J. Cho, and J. M. DeSimone, "Nanofabricated particles for engineered drug therapies: A preliminary biodistribution study of PRINT ${ }^{\mathrm{TM}}$ nanoparticles," Journal of Controlled Release, vol. 121 , no. 1-2, pp. 10-18, 2007.

[26] Y. C. Saraswat, F. Ibis, L. Rossi, L. Sasso, H. B. Eral, and P. Fanzio, "Shape anisotropic colloidal particle fabrication using 2-photon polymerization," Journal of Colloid and Interface Science, vol. 564, pp. 43-51, 2020.

[27] I. Zein, D. W. Hutmacher, K. C. Tan, and S. H. Teoh, "Fused deposition modeling of novel scaffold architectures for tissue engineering applications," Biomaterials, vol. 23, no. 4, pp. 1169-1185, 2002.

[28] A. Bertsch, H. Lorenz, and P. Renaud, "3D microfabrication by combining microstereolithography and thick resist UV lithography," Sensors and Actuators, A: Physical, vol. 73, no. 1-2, pp. 14-23, 1999.

[29] S. Badaire, C. Cottin-Bizonne, J. W. Woody, A. Yang, and A. D. Stroock, "Shape selectivity in the assembly of lithographically designed colloidal particles," Journal of the American Chemical Society, vol. 129, no. 1, pp. 40-41, 2007.

[30] C. J. Hernandez and T. G. Mason, "Colloidal alphabet soup: Monodisperse dispersions of shape-designed LithoParticles," Journal of Physical Chemistry $C$, vol. 111, no. 12, pp. 4477-4480, 2007.

[31] W. D. Ristenpart, I. A. Aksay, and D. A. Saville, "Electrohydrodynamic flow around a colloidal particle near an electrode with an oscillating potential," Journal of Fluid Mechanics, vol. 575, pp. 83-109, 2007.

[32] F. Ma, X. Yang, H. Zhao, and N. Wu, "Inducing propulsion of colloidal dimers by breaking the symmetry in electrohydrodynamic flow," Physical Review Letters, vol. 115, no. 20, pp. 1-5, 2015.

[33] X. Yang and N. Wu, "Change the collective behaviors of colloidal motors by tuning electrohydrodynamic flow at the subparticle level," Langmuir, vol. 34, no. 3, pp. 952-960, 2018.

[34] W. Wang, X. Lv, J. L. Moran, S. Duan, and C. Zhou, "A practical guide to active colloids: choosing synthetic model systems for soft matter physics research," Soft Matter, vol. 16, no. 16, pp. 3846-3868, 2020. 



\section{Summary}

Colloids are small particles that are roughly between $1 \mathrm{~nm}$ and $1 \mu \mathrm{m}$ in size. At the upper size range these particles are small enough to display significant Brownian motion due to thermal fluctuations while large enough to be easily visible using relatively simple and affordable optical microscopes. These properties make colloids very suitable to use in model systems.

In the first part of this thesis we study the lattice structures in monolayers of colloidal superballs prepared using the unidirectional rubbing method. The superball shape is an interpolation between the spherical and cubic shape. Their shape is characterized using the shape parameter $m$ which ranges from $m=2$ for spheres to $m \rightarrow \infty$ for cubes. The non-spherical superballs studied in this thesis were prepared by coating colloidal hematite cubes with a layer of silica using a modified Stöber method. The magnetic hematite cores were subsequently dissolved with hydrochloric acid. By coating colloidal hematite cubes of various sizes with various thicknesses of silica, these colloidal superballs can be prepared in a wide size range with edge diameters $D$ of around between 500 and $1500 \mathrm{~nm}$ and with shape parameters $m$ of around between 2 and 4 . In order to assemble the colloidal silica superballs into monolayers reproducibly using the unidirectional rubbing method we have designed and constructed a custom-made prototype device. This open-source device uses mechanical force to rub the dried colloids onto polydimethylsiloxane- (PDMS) coated glass slides using a PDMS cylinder. This device allows the rubbing speed, pressure and direction to be precisely controlled. These experimental methods were described in detail in chapter 2 .

In chapter 3 we have presented a measurement and analysis strategy for studying the monolayers of colloidal superballs assembled using the unidirectional rubbing method with small-angle X-ray scattering (SAXS). SAXS is a very capable analysis technique to study the long-range ordering of crystals in both two and three dimensions. In the resulting diffraction patterns the measured intensity is the product of the form factor and the structure factor. These diffraction patterns can be difficult to analyze as not all Bragg peaks may appear due to the presence of minima in the form factor. For spheres the form factor is isotropic and can be calculated analytically. For hollow superballs, however, the form factor is a function of the size, shape and thickness of the silica shell which cannot be calculated analytically and can thus make the diffraction patterns unpredictable. To visualize these missing Bragg peaks we have imaged the colloidal monolayers under various rotations around the $x$-axis, which was the direction the mechanical rubbing was applied in. Using three superball shapes, spherical, semi-cubic and cube-like, we show that the missing Bragg peaks can be visualized by imaging the samples over a range of rotations, resulting in a complete and more accurate analysis. We have 
observed a transition from the hexagonal close-packed lattice to the so-called sliding phase upon increasing the shape parameter $m$ of the superballs. In the sliding phase chains of cube-like superballs form in the direction in which the mechanical rubbing was applied. These chains are able to slide with respect to each other to some degree depending on the shape of the superball particles. The exact degree of sliding could not be determined with SAXS alone, however.

In chapter 4 we continued to study the lattice structures in monolayers of colloidal superballs assembled using the unidirectional rubbing method. In addition to SAXS, we have employed scanning electron microscopy (SEM) to study the local order in the lattice structures. Using a custom-written tracking script, the positions and orientations of the colloidal superballs in the lattices were determined. Different data can be extracted from the samples with SEM compared to SAXS and using a combination of SAXS and SEM we were able to perform a complete analysis of the superball lattice structures. We show using both spherical and cube-like colloidal superballs that the unidirectional rubbing method is a very suitable method for the assembly of superball-shaped colloids into lattice structures. The resulting superball lattice structures had a very well-defined ordering over a wide range of rubbing speeds and pressures, with the more cubic superballs requiring a slightly higher rubbing pressure compared to the spherical superballs. Using various shapes and sizes of colloidal superballs we studied the influence of the superball shape and size on the resulting lattice structures. Using SAXS we found that the ratio $a_{1} / a_{2 x}$, which describes the ratio between the vertical distance between adjacent chains of superballs formed in the horizontal direction to the interparticle distance between the colloids in these horizontal chains, increases upon increasing the shape parameter $m$ of the superballs as expected. For all superball shapes and sizes the lattice structures aligned strongly in the direction of the applied rubbing motion. Both primitive basis vectors $a_{1}$ and $a_{2 x}$ were around 5 to $10 \%$ higher compared to those for an ideal close-packed lattice of superballs. This amount of empty space present between the colloidal superballs is not surprising as there are no attractions present between the particles during their assembly using the unidirectional rubbing method. De degree at which the chains of superballs in the horizontal direction are able to slide with respect to each other was determined from the SEM images using the two-dimensional density distributions. Here, the degree at which these chains are able to slide with respect to each other was found to increase continuously upon increasing the shape parameter $m$ of the colloidal superballs. At a shape parameter of around $m=3.5$ these chains become completely free to slide with respect to each other, resulting in random shifts in their lattice structures. Thus, a continues transformation from the hexagonal lattice to the sliding phase occurs upon increasing the shape parameter $m$ of the colloidal superballs. 
In chapter 5 we have described the behaviour of active colloidal circlers, active colloids that are self-propelled into circular trajectories, powered by alternating electric fields. These active colloids were prepared by first assembling colloidal PMMA spheres with an average diameter of $4.0 \mu \mathrm{m} \pm 2.5 \%$ into colloidal monolayers using the unidirectional rubbing method. The resulting colloidal monolayers were then coated with a $15 \mathrm{~nm}$ thick layer of gold under various glancing angles $\theta$. The well-defined periodicity in the two dimensional lattice structures as well as the alignment of the colloidal crystal grains in the direction in which the mechanical rubbing was applied make the unidirectional rubbing method ideal for the preparation of active colloids. The relatively low polydispersity of these colloids in combination with the use of the unidirectional rubbing should minimize the polydispersity in the size, shape and thickness of the sputter coated gold patch, which should therefore minimize the variation in the behaviour of the resulting active colloidal circlers. These active colloids become self-propelled under the influence of an alternating electric field due to induced-charge electrophoresis (ICEP). By coating the colloidal monolayers under a glancing angle, the sputter coated gold patch can be made asymmetrical, which was observed to result in the active colloids self-propelling in circular trajectories. Here, upon increasing the glancing angle of sputter coating, the radii in which these active colloids were observed to circle decreased. The velocity of the active colloidal circlers, on the other hand, only decreased slightly upon increasing the glancing angle. These active colloidal circlers are effectively chiral in two dimensions as the alternating electric field pins the gold patch along the vertical direction and, as a result, are unable to switch their direction of circling. The polydispersity in the velocity and angular velocity of these particles were around 15 and $30 \%$ respectively for all glancing angles, which can be considered quite low due to the known sensitivity of ICEP to the shape and thickness of the applied metal patch. Furthermore, we have studied the influence of both the magnitude and frequency of the alternating electric field on the behaviour of these active colloidal circlers. We found that upon increasing the magnitude of the alternating electric field both the velocity and angular velocity of the particles increased quadratically with the radii of the resulting circular trajectories remaining constant. Upon increasing the frequency of the alternating electric field the angular velocity increased while the velocity decreased with the radii of the resulting circular trajectories decreasing. Thus, by varying both the magnitude and frequency of the applied alternating electric field both the velocity and angular velocity can be tuned in situ. These particles may thus be suitable as sensors for alternating electric fields as a certain combination of frequency and magnitude corresponds to a certain combination of a velocity and angular velocity and vice versa. Finally, these active colloidal circlers are promising for self-assembly studies, where the circling motion might result in 
the formation of novel active phases. We also introduced a modification in the synthesis method of these particles to virtually eliminate the remaining velocity. This was done by coating the colloidal spheres on both hemispheres with a patch of platinum instead. The resulting active colloidal spinners have inversion symmetry and only display rotational motion without significant translational motion. Under the influence of an alternating electric field these active colloidal spinners were observed to rotate with an average frequency of around $0.1 \mathrm{~s}^{-1}$. The relatively slow rotation might be increased by further optimizing the synthesis method. In the literature these type of particles have been shown to be useful as colloidal stirrers and have been shown to be able to assist self-assembly processes.

In chapter 6 we have presented a novel synthesis method for the preparation of active anisotropic hydrogel partices. These hydrogel particles were prepared by first spin coating glass slides with a thin film of poly(ethylene glycol) diacrylate (PEGDA) containing a photoinitiator. The glass slides were first hydrophilyzed by leaving them in a Piranha solution for 30 minutes. As a result of the hydrophilization, the spin coated PEGDA films are stable for several hours. During this time the hydrogel particles can be prepared by locally polymerizing the PEGDA film using UV light. Here, the shape of the mask placed at the field stop of the optical microscopy determines the in-plane shape and size whereas the thickness of the the PEDGA film determines the thickness of the resulting hydrogel particles. This method allows the production of hydrogel particles at a rate of around 10.000 particles per hour. We show that by varying the spin coating time, while keeping the spin coating speed constant at $1000 \mathrm{rpm}$, hydrogel particles with various thicknesses ranging from around 10 to $35 \mu \mathrm{m}$ can be prepared. Due to the nature of this preparation method, the particles are rounded on top due to being exposed to air. Here, oxygen quenches the polymerization of the exposed PEDGA layer. This effect, however, becomes less noticeable for larger particles. This method is easy to use and requires no special equipment besides an optical microscope equipped with a motorized stage and an UV light source and should therefore be accessible to a broad audience. Finally, we briefly show that these particles display active motion under the influence of an alternating electric field. Albeit quite slow, these anisotropic hydrogel particles become self-propelled as a result of their asymmetric shape due to induced electrohydrodynamic (EDH) flows. We have observed that these hydrogel particles, however, also unexpectedly self-propelled into circular trajectories with various radii, possibly due to the presence of defects in the geometry of these hydrogel particles. While more research is thus required to improve the design of these active colloids to obtain active particles with well-defined motion, these active hydrogel particles may be useful for studying the self-assembly of active particles with arbitrary shapes. 


\section{Samenvatting voor Iedereen}

In deze "Samenvatting voor Iedereen" is het onderzoek beschreven in dit proefschrift beknopt samengevat in simpele taal. Zo zijn de meest interessante resultaten en de belangrijkste conclusies die dit onderzoek heeft opgeleverd toegankelijk voor iedereen.

Het hoofdonderwerp van dit proefschrift zijn de zogenaamde colloïden. Colloïden zijn miniscule deeltjes ter grootte van ongeveer 0.001 millimeter, wat ongeveer één honderdste van de dikte van een menselijke haar is. Ze zijn daardoor dus veel te klein om met het blote oog te kunnen zien. Colloïden zijn overal te vinden zoals in bijvoorbeeld melk, inkt, kaas en mist. Daarnaast kunnen colloïden gebruikt worden voor het maken van modellen. Een model is een vereenvoudigde weergave van de werkelijkheid waarin slechts de belangrijkste elementen beschouwd worden. Modellen worden gebruikt om complexe processen te kunnen begrijpen en te kunnen voorspellen, variërend van bijvoorbeeld het weer tot economische ontwikkelingen. In dit proefschrift beschrijven we enkele door ons ontwikkelde colloïdale model systemen. Colloïdale model systemen kunnen inzicht geven in chemische of fysische processen, wat kan leiden tot nieuwe technologische ontwikkelingen.

In het eerste gedeelte van dit proefschrift bestuderen we colloïden met de zogenaamde superbal vorm. De superbal vorm beschrijft de transformatie van de vorm van bollen naar kubussen. De vorm van deze superbalvormige deeltjes wordt beschreven met behulp van de zogenaamde $m$-waarde. Deze $m$-waarde is $m=2$ voor bollen en $m \rightarrow \infty$ ( $m$ wordt oneindig groot) voor kubussen. Schematische voorbeelden van superbal deeltjes met verschillende vormen en hun $m$-waarden zijn weergegeven in figuur 1 .

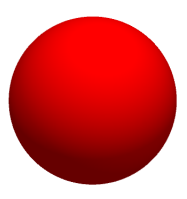

$$
m=2
$$

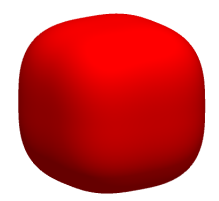

$m=3$

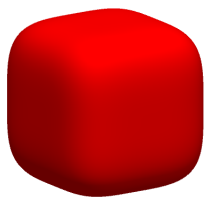

$m=4$

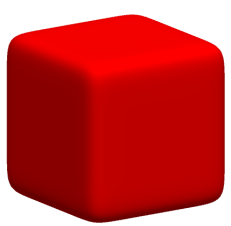

$m=10$

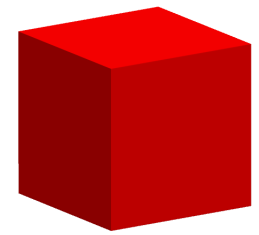

$m \rightarrow \infty$

Figuur 1: Schematische voorbeelden van superbal deeltjes met verschillende $m$ waarden variërend van $m=2$ voor bollen tot $m \rightarrow \infty$ ( $m$ wordt oneindig groot) voor kubussen.

In het lab zijn deze colloïdale superballen te maken met veschillende $m$ waarden tussen ongeveer $m=2$ en 4 en met een diameter van tussen ongeveer 0.0005 en 0.0015 millimeter. Doordat deze deeltjes gemaakt kunnen worden met verschillende vormen en groottes kan de invloed van de vorm en grootte van deze deeltjes in of op een bepaald proces bestudeerd worden. In dit proef- 
schrift hebben we de invloed van de superbal vorm en hun grootte op de structuur van de resulterende twee-dimensionale kristalroosters bestudeerd. Een kristalrooster bestaat uit een groot aantal deeltjes welke zijn geordend in een regelmatig patroon. Wij hebben deze twee-dimensionale kristalroosters gemaakt door de colloïdale superballen over een glasplaatje met een plakkerige coating te schuiven, de zogenaamde schuifmethode. Het schuiven werd machinaal gedaan om zo het schuiven op een gecontroleerde manier uit te kunnen voeren met een bepaalde druk en snelheid. Op deze manier kan ook de invloed van de druk en snelheid van het schuiven op de structuur van de kristalroosters bepaald worden. We hebben deze kristalroosters van colloïdale superballen bestudeerd met zowel small-angle X-ray scattering (SAXS) en scanning electron microscopy (SEM). Deze twee verschillende technieken vullen elkaars zwakheden perfect op, wat de analyse van de kristalroosters zo compleet mogelijk maakt.

Een scanning elektronenmicroscoop maakt een foto, vergelijkbaar met een normale camera, maar gebruikt hiervoor elektronen in plaats van zichtbaar licht. Door gebruik te maken van elektronen kunnen plaatjes met een zeer grote resolutie gemaakt worden, waardoor details zichtbaar worden die niet met normale camera's te zien zouden zijn. Representatieve SEM plaatjes van drie voorbeelden van twee-dimensionale kristalroosters van colloïdale superballen met $m$-waarden van $m=2,3$ en 4 en met een diameter van ongeveer 0.001 millimeter zijn weergegeven in figuur $2 \mathrm{a}$, b en c.
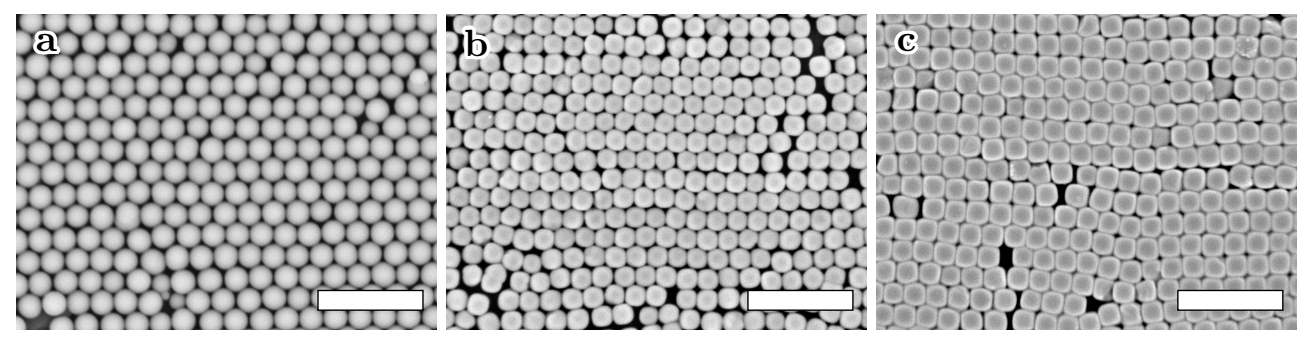

Figuur 2: Representatieve SEM plaatjes van kristalroosters van colloïdale superballen met $m$-waarden van $m=2$ (a), 3 (b) en 4 (c). De schalen weergegeven in de plaatjes zijn 0.005 millimeter.

Voor de bolvormige colloïdale superballen $(m=2)$, weergegeven in figuur $2 \mathrm{a}$, is het te zien dat de deeltjes zijn geordend in een hexagonaal rooster. Hier heeft elk deeltje zes andere deeltjes om zich heen op precies dezelfde posities. Voor de bijna kubusvormige colloïdale superballen met een $m$-waarde van $m=4$, weergegeven in figuur 2c, is het te zien dat deze deeltjes zich in rijen in de horizontale richting ordenen, de richting waarin de deeltjes geschoven werden tijdens het maken. De verschuivingen tussen deze rijen lijken willekeurig doordat de deeltjes zich lokaal in zowel vierkante als hexagonale roosters kun- 
nen ordenen. Tussen deze twee superbalvormen in zitten de colloïdale superballen met een $m$-waarde van $m=3$ zoals weergegeven in figuur $2 \mathrm{~b}$. Dit kristalrooster heeft kenmerken die tussen de andere twee kristalroosters in vallen. Hier zijn de superbal deeltjes vooral hexagonaal geordend, maar er zijn hier ook kleine verschuivingen tussen de rijen deeltjes in de horizontale richting zichtbaar.

Omdat SEM erg ingezoomd op het sample, is slechts een klein deel van het sample te zien in elk van de plaatjes. Zelfs met tientallen plaatjes per sample is het moeilijk te zeggen of deze data representatief is voor het gehele sample. Daarom hebben we ook SAXS gebruikt voor de analyse van deze kristalroosters. SAXS meet namelijk data over een veel groter gedeelte van het sample, waardoor de gemiddelde eigenschappen betrouwbaar gemeten kunnen worden. Met deze techniek wordt het sample beschenen met een zeer intense röntgenstraal. Een gedeelte van deze röntgenstraling wordt door het sample verstrooit, dit kan worden gezien als het botsen en afketsen van de röntgenstraling op het sample. Van deze verstrooide röntgendeeltjes wordt de intensitieit op de detector, wat als het ware een camera is voor röntgenstraling, gemeten. Het resulterende plaatje wordt het diffractiepatroon genoemd. De bijbehorende diffractiepatronen van de hierboven bediscussieerde kristalroosters van colloïdale superballen met $m$ waarden van $m=2,3$ en 4 zijn weergegeven in figuur $3 \mathrm{a}, \mathrm{b}$ en $\mathrm{c}$. Uit de ordering en de positie van de pieken in het diffractiepatroon kan de structuur van het kristalrooster van het sample achterhaald worden.
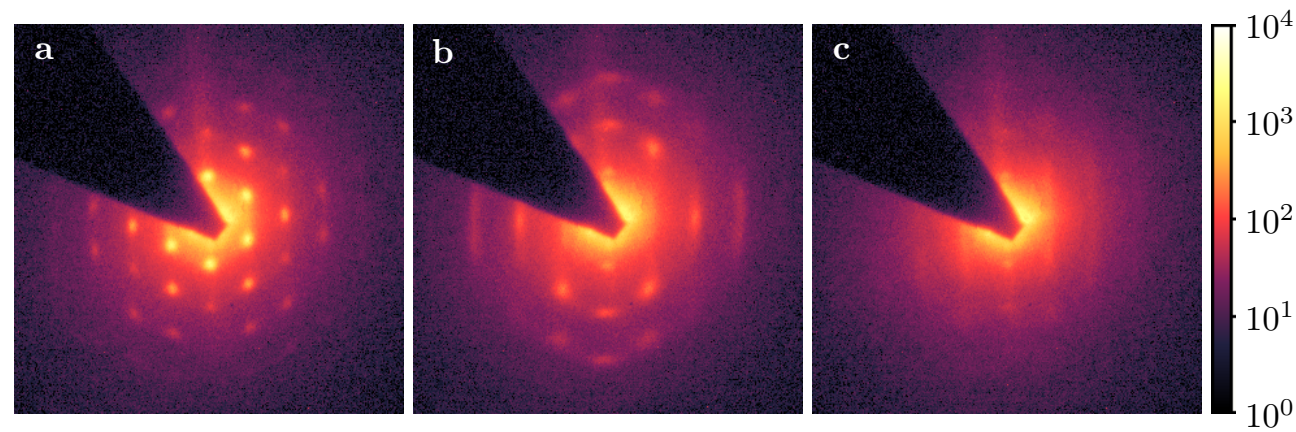

Figuur 3: De bijbehorende diffractiepatronen van de eerder beschreven kristalroosters van colloïdale superballen met $m$-waarden van $m=2$ (a), 3 (b) en 4 (c).

Uit zowel de SEM als de SAXS data en analyse blijkt dat de transitie in de kristalroosters vanuit de hexagonale structuur voor bollen naar de zogenaamde schuifstructuur voor de bijna kubusvormige superballen continue is. Een verhoging van de $m$-waarde van de colloïdale superballen leidt hier dus tot een geleidelijke verhoging van de mate waarin de rijen deeltjes in de horizontale richting langs elkaar heen kunnen schuiven. In tegenstelling tot de 
vorm heeft de grootte van de colloïdale superballen geen significante invloed op de structuur van het kristalrooster. Daarnaast hebben we gevonden dat er een minimale hoeveelheid druk nodig is waarmee de superbalvormige deeltjes over de plakkerige coating worden geschoven worden om de kristalroosters te kunnen vormen. Boven deze minimale druk kunnen deze kristalroosters reproduceerbaar gemaakt worden over een breed bereik van druk en snelheid. Uit de bovenstaande observaties kan worden geconcludeerd dat de schuifmethode een zeer geschikte methode is voor het maken van kristalroosters van colloïdale superballen. Deze kristalroosters zouden in de toekomst gebruikt kunnen worden in toepassingen zoals bijvoorbeeld in coatings en sensoren.

Door de duidelijke structuur in de resulterende kristalroosters en hun uitlijning in de schuifrichting is de schuifmethode ook zeer geschikt als tussenstap in de productie van actieve colloïden. Actieve colloïden zijn colloïden welke een chemische of fysische brandstof verbruiken om zichzelf te verplaatsen. Deze verplaatsingen worden de actieve beweging genoemd.

In het tweede deel van dit proefschrift beschrijven we een type van deze actieve colloïden. Deze actieve colloïden hebben wij gemaakt door de kristalroosters van bolvormige colloïden, soortgelijk als afgebeeld in figuur $2 \mathrm{a}$, te coaten met een dun laagje goud van ongeveer 0.00001 millimeter dik. Dit dunne laagje goud was slechts ongeveer 100 goud atomen dik. Na het coaten met goud werd het kristalrooster uit elkaar gehaald, waarna de individuele actieve beweging van de deeltjes bestudeerd kon worden. Het kristalrooster wordt hier dus gebruikt om de goudcoating een bepaalde vorm te geven, net zoals bijvoorbeeld afplaktape gebruikt kan worden tijdens het schilderen. We hebben gevonden dat wanneer dit goudlaagje wordt aangebracht door van bovenaf te coaten, deze coating uniform zal zijn op de bovenkant van de colloïden in het kristalrooster. Door deze uniforme coating zullen de resulterende actieve colloïden zichzelf rechtuit voortbewegen zoals afgebeeld in figuur 4a. Rechtsboven in dit plaatje is het actieve deeltje te zien als een klein zwart stipje. De afgelegde route is hier in het rood getekend. Doordat de coating uniform is zal het deeltje geen voorkeur hebben voor links of rechts en zichzelf dus rechtuit voortbewegen. Wanneer dit goudlaagje onder een hoek op de colloïdale kristalroosters wordt aangebracht zal deze coating niet uniform zijn op de bovenkant van de colloïden in het kristalrooster. We zien dan dat de resulterende actieve colloïden zichzelf in cirkelvormige banen voortbewegen zoals afgebeeld in figuur $4 \mathrm{~b}$ en c. Deze deeltjes werden onder een hoek van 20 en 40 graden gecoat. Het is hier ook duidelijk te zien dat wanneer de hoek waarmee dit goudlaagje op de colloïdale kristalroosters wordt aanbracht vergroot wordt, de straal van de cirkelvormige banen afneemt. Het gedrag van de resulterende actieve colloïden kan dus worden "geprogrammeerd" door de hoek tijdens het coaten met goud te variëren. 

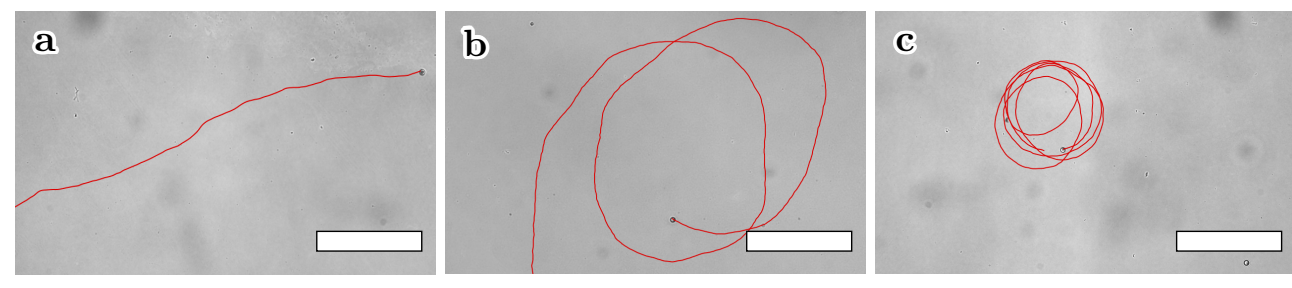

Figuur 4: Representatieve voorbeelden van actieve colloïden met hun afgelegde route in het rood getekend. Deze actieve collö̈den zijn gemaakt door de collö̈dale kristalroosters te bedekken met een laagje goud aangebracht vanaf 0 graden (bovenaf) (a), 20 graden (b) en 40 graden (c). Door de colloïdale kristalroosters onder een hoek te coaten ontstaat een niet-uniforme goudlaag waardoor de actieve deeltjes zich in cirkelvormige banen gaan voorbewegen in plaats van rechtuit. De schalen weergegeven in de plaatjes zijn 0.05 millimeter.

De brandstof die in onze experimenten gebruikt werd om de actieve colloïden voort te bewegen waren elektrische wisselvelden. Deze elektrische wisselvelden ontstonden doordat het celletje waarin de deeltjes bestudeerd werden onder een elektrische stroom stond. Ook hebben we de invloed van de sterkte en frequentie van het elektrische wisselveld op het gedrag van deze deeltjes bestudeerd. We hebben gevonden dat een verhoging van de sterkte van het elektrische wisselveld leidt tot een snellere actieve beweging van deze deeltjes, maar dat de straal waarin de deeltjes cirkelden constant bleef. Bij het verhogen van de frequentie van het elektrische wisselveld was te zien dat de deeltjes zich minder snel voortbewogen, en dat ook de straal van de cirkelvormige banen afnam. De invloed van de sterkte en de frequentie van het elektrische wisselveld op het gedrag van de deeltjes maakt ze geschikt om zowel de sterkte als de frequentie van een aangelegd elektrisch wisselveld te meten. In de toekomst zouden deze actieve colloïdalen dus mogelijk gebruikt kunnen worden als sensoren voor elektrische wisselvelden. Tevens beschrijven we een meer complexe variant van dit type actieve colloïden waar de beweging bijna compleet uitschakeleld is. Deze variant draait enkel rond en blijft dus vrijwel op zijn plek stil staan. Deze actieve colloïden kunnen bijvoorbeeld worden gebruikt om vloeistoffen zeer lokaal te roeren.

Ten slotte beschrijven we in het laatste hoofdstuk van dit proefschrift de ontwikkeling van een methode om nog een ander type deeltjes te kunnen maken. Hier beschijnen we een glasplaatje welke een dun laagje vloeistof bevat met een korte, felle puls van UV licht. Dit laagje met een dikte van ongeveer 0.01 millimeter hardt uit wanneer deze beschenen wordt met het UV licht. Door een bepaalde vorm te geven aan dit UV licht kunnen deeltjes met die specifieke vorm gemaakt worden. Deze deeltjes worden één voor één geprint in roosters zoals weergegeven in figuur 5a. Voorbeelden van deze geprinte deeltjes met verschillende vormen zijn weergegeven in figuur $5 \mathrm{~b}, \mathrm{c}, \mathrm{d}$ en e. 

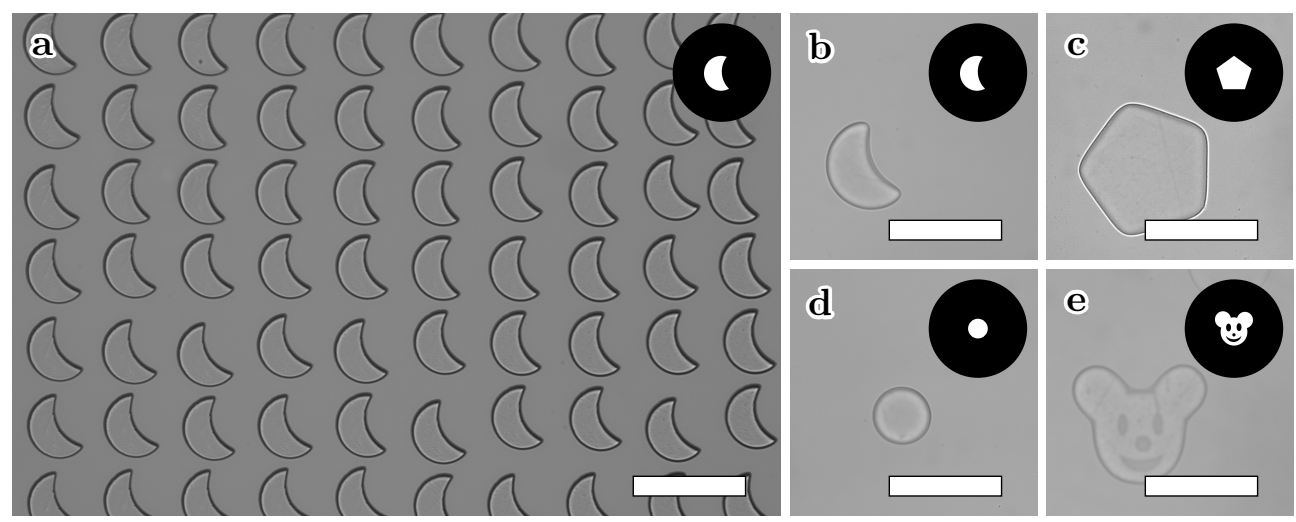

Figuur 5: De deeltjes worden één voor één geprint door een glasplaatje welke een dunne vloeibare laag bevat te beschijven met een puls van UV licht (a). Een masker wordt gebruikt om de vorm van het UV licht, en dus de vorm van het uiteindelijke deeltje te bepalen (b-e). De vorm van het gebruikte masker zijn weergegeven in elk van de plaatjes. De schalen weergegeven in de plaatjes zijn 0.1 millimeter.

Zoals te zien is aan de schalen in elk van de plaatjes zijn deze type deeltjes veel groter dan de eerder bediscussieerde actieve cirkelende deeltjes en de colloïdale superballen. Dit komt door het gebruik van UV licht, welke een eindige resolutie heeft, en dus een limiet geeft in hoe klein de deeltjes gemaakt kunnen worden. De dikte van de resulterende deeltjes wordt bepaald door de dikte van de vloeibare laag die op het glasplaatje is aangebracht. Voor het reproduceerbaar maken van dit type deeltjes is geen speciale kennis of apparatuur nodig, alleen een microscoop met een UV lichtbron, wat deze methode erg toegankelijk maakt. Ook deze deeltjes kunnen een actieve beweging vertonen in elektrische wisselvelden wanneer deze deeltjes een asymmetrische vorm hebben. Deze relatief grote en simpele actieve deeltjes kunnen in de toekomst mogelijk gebruikt worden voor de assemblage van meer complexe structuren. 


\section{Dankwoord}

Onderzoek doen doe je niet alleen en de afgelopen vier jaar hebben dan ook vele collega's, studenten, vrienden en familie op de één of andere manier bijgedragen aan dit onderzoek. Hierbij wil ik dan ook iedereen graag bedanken voor zijn of haar bijdrage aan het tot stand komen van dit proefschrift.

Andrei, allereest wil ik jou graag bedanken voor de kans die je mij hebt gegeven om samen aan dit avontuur te beginnen en voor de vrijheid die je mij hebt gegeven om zelf het onderzoek in te kunnen delen. Als dagelijkse begeleider was jij het dichtst bij dit project betrokken en je deur stond ook altijd voor mij open. Jouw enthousiasme voor SAXS is geweldig groot en dit werkt ook aanstekelijk. Ik ben dan ook blij dat SAXS een groot onderdeel van dit onderzoek is geworden. In Grenoble kwam je helemaal tot leven en het werken daar is voor mij ook een ervaring geweest om nooit te vergeten.

Uiteraard wil ik jou Albert, mijn promotor, ook graag bedanken voor je hulp. Jouw aandacht voor details alsook je nuchtere blik op de wetenschap, met name op het experimentele werk en dat dit niet altijd loopt zoals gepland of gehoopt, hebben mij enorm geholpen. Ook werkte ik graag met je samen tijdens het assisteren van de werkcolleges van Fysische Chemie 1 en Colloid Science.

Janne-Mieke, ik wil jou graag bedanken voor je hulp en je uitgebreide inzicht op het gebied van colloïdale superballen en hun assemblage in monolagen. Deze samenwerking heeft mij enorm geholpen met de analyse van de data en het schrijven van de hoofdstukken. Burak, ook jou wil ik graag bedanken voor de nuttige discussies en je inzicht in de verschillende lithografie technieken. Ook deze samenwerking heeft mij veel geholpen. Ivan, I have enjoyed your ever-positive attitude towards life and science and your presence as a roommate in the office. I really liked working together with you on making the do-it-yourself Arduino-powered microscope stage.

Geen enkel lab kan zonder goed ondersteunend personeel. Dominique, door jou harde werk liep het lab van FCC zo goed als het deed. Ik wil jou niet alleen hiervoor graag bedanken, maar ook voor je hulp met de synthese van de colloïdale silica bollen en voor je suggesties om het hoofd koel te houden wanneer het weer even niet lukt zoals gepland. Ook wil ik graag Kanvaly en Bonny bedanken voor hun technische ondersteuning en Marina voor de administratieve ondersteuning. Alex, ook jou wil ik bedanken voor je rol in de technische ondersteuning. Ik wens je veel succes met je nieuwe functie. Daarnaast wil ik Hans en Chris van het elektronenmicroscopie lab graag bedanken voor hun vele suggesties, troubleshooting en voor hun gastvrijheid tijdens de talloze bezoekjes voor de elektronenmicroscopen en vooral de sputter coater. Ik wil Regina graag bedanken voor het schoonhouden van de labs en het kantoortje. 
In het kantoortje hing altijd een gezellige en ontspannen sfeer. Hier wil ik mijn oud-kamergenoten Ivan, Álvaro, Michele, Azeem, Mariska en Raimon voor bedanken. Ook wil ik graag de rest van FCC bedanken. Amanda, Alessio, Ben, Didier, Faranaaz, Frans, Fuqiang, Jan, James, Jasper, Joren, Marieke, Martin, Neshat, Pepijn, Ping, Willem, Xiuhang, Yong en alle bachelor- en masterstudenten, bedankt. Bedankt voor de gezellige sfeer tijdens het werk, de pauzes en alle uitjes de afgelopen jaren.

Ik heb het geluk gehad om met veel studenten te mogen samenwerken gedurende dit project. Hoewel niet al jullie harde werk terug te vinden zal zijn in dit proefschrift wil ik jullie toch allemaal hartelijk bedanken voor jullie inzet. Jan, Paul, Ties en Laura, mijn eerste groepje tweedejaars studenten, hebben in hun researchproject gewerkt aan het maken van katalytische actieve colloïden welke voortbewegen door waterstofperoxide te ontbinden. Dit was nog in het beginstadium van het onderzoek naar de cirkelende actieve colloïden. Jarno en Steijn hebben voor hun profielwerkstuk geholpen aan de verdere ontwikkeling van deze cirkelende actieve colloïden, nu voortbewogen met behulp van elektrische wisselvelden, net zoals Annemiek in haar bachelorthesis. Jesse, Floris, Frank en Wessel hebben geholpen met het maken van hydrogel deeltjes welke colloïdaal goud bevatten door middel van stop-flow lithografie tijdens hun researchproject. Gelijktijdig hebben Ffion, Friso, Kyra en Sander geholpen met de ontwikkeling van de stilstaande, draaiende actieve colloïden. Florine is later verder gegaan met het ontwikkelen van actieve hydrogel deeltjes gemaakt door middel van stop-flow lithografie en het doorspitten van de literatuur als onderdeel van haar bachelorthesis. Kasper heeft tijdens zijn bachelorthesis meegeholpen aan het ontwikkelen van actieve hydrogel deeltjes gemaakt door middel van lithografie. Ten slotte hebben Casper en Jaap geholpen met het bestuderen van de cirkelende actieve colloïden in elektrische wisselvelden bij hoge concentraties als onderdeel van hun profielwerkstuk. Ik vond het leuk om jullie allen te begeleiden en ik heb hier zelf ook veel van geleerd. Hopelijk kijken jullie ook met vreugde terug op deze tijd.

Ik wil mijn vriendenkring bedanken voor alle lol en afleiding de afgelopen jaren. Joeri, ik ben blij dat je mij ook bij wilt staan als paranimf. Mijn familie, mama en Fred, papa en Irene, opa en oma en mijn broertjes Etienne en Harm, wil ik graag bedanken voor al hun steun en gezelligheid. Mijn schoonfamilie, Freddy en Joke, wil ik bedanken voor hun steun en gastvrijheid. Bij jullie kwam je nooit ongelegen en was het altijd gezellig. Ten slotte wil ik jou Roswitha, het allerliefste schatje, bedanken voor al je steun, liefde en lol de afgelopen jaren, en dat je mij ook bij wilt staan als paranimf. Met jou wordt alles leuk. 


\section{List of Publications}

This thesis is based on the following publications:

- D. N. ten Napel, J.-M. Meijer and A. V. Petukhov, "The Analysis of Periodic Order in Monolayers of Colloidal Superballs", submitted. (Chapter 3)

- D. N. ten Napel, J.-M. Meijer and A. V. Petukhov, "The Mechanical Assembly of Colloidal Superballs", in preparation. (Chapter 4)

- D. N. ten Napel, A. P. Philipse and A. V. Petukhov, "Active Colloids with Circling Motion Powered by Alternating Electric Fields", in preparation. (Chapter 5)

- D. N. ten Napel, K. J. Tempel, H. B. Eral and A. V. Petukhov, "Production of Active Anisotropic Colloidal Hydrogel Particles", in preparation. (Chapter 6)

Other publications by the author:

- Y. N. Vakkipurath Kodakkadan, K. Idzakovicova, J. Sepitka, D. ten Napel, E. Safai, P. Cigler, F. Stepanek, and I. Rehor, "ArbitrarilyShaped Microgels Composed of Chemically Unmodified Biopolymers", Biomaterials Science, vol. 8, no. 11, pp. 3044-3051, 2020. 



\section{About the Author}

Daniël ten Napel was born on March 25, 1991 in Amsterdam, the Nederlands. He graduated from VMBO at the Bernard Nieuwentijt College in Monnickendam in 2007, from HAVO at the Oosterlicht College in Nieuwegein in 2009 and from VWO at the Oosterlicht College in Nieuwegein in 2011. After finishing high school, he started the bachelor Chemistry at Utrecht University where he obtained his bachelor's degree cum laude in 2014. He wrote his bachelor thesis at the Condensed Matter and Interfaces group under the supervision of Tim Senden and prof. dr. Andries Meijerink on the topic of synthesizing modified cerium-doped yttrium aluminum garnets in order to obtain a red-shift in their emission spectra. Afterwards, he started the master Nanomaterials: Chemistry and Physics at Utrecht University. He wrote his master thesis at the Van 't Hoff Laboratory for Physical and Colloid Chemistry under the supervision of Ping Liu and prof. dr. Albert Philipse on the topic of the self-assembly of magnetic colloids at liquid-liquid interfaces with added depletion interactions in order to make structural patterns in two-dimensions, the so-called stripe phase. He finished his master with an internship at AMOLF in Amsterdam under the supervision of dr. Wim Noorduin on the topic of developing methods for chiral resolution. The goal of this project was to use the formation of Liesegang rings, a famous reaction-diffusion system, to separate the enantiomers of racemic mixtures. He obtained his master's degree cum laude in 2016. Afterwards, he started his $\mathrm{PhD}$ project in October 2016 under the supervision of dr. Andrei Petukhov and prof. dr. Albert Philipse at the Van 't Hoff Laboratory for Physical and Colloid Chemistry. The most interesting results of this research are described in this thesis. 
